\title{
2017 KASL clinical practice guidelines management of hepatitis C: Treatment of chronic hepatitis C
}

\author{
The Korean Association for the Study of the Liver (KASL)*
}

Keywords: Chronic hepatitis C; Treatment; Direct acting antivirals; Guidelines

\section{INTRODUCTION}

Guidelines on the management of hepatitis $C$ were first developed in 2004 and revised in 2013 and 2015 by the Korean Association for the Study of the Liver (KASL). Recently, paradigms for the treatment of chronic hepatitis $C$ have changed from interferon (IFN) alpha-based treatment to direct-acting antiviral agents (DAA). Compared with IFN, the higher sustained virologic response rate (SVR) of around 95\%, lower adverse events, and convenience of DAAs for hepatitis C approaches the ideal goals of antiviral treatment. However, treatment for individual patients remains complex because treatment regimens and durations can differ depending on previous treatment experience, virus geno- type and subtype, and the presence or absence of underlying liver cirrhosis. Furthermore, re-treatment options for patients who failed on previous DAA are limited. Since the first release of DAA, there have been many developments, including the introduction of pan-genotypic DAAs, new antivirals against resistance-associated substitutions (RASs), and the publication of many novel research results from Korea and other countries.

Therefore, this clinical practice guideline, Management of hepatitis C: Treatment of chronic hepatitis $C$, has been revised in the areas of treatment, research, and education. These recommendations are not absolute standards of care, and adoption of the guidelines in clinical practice might need to differ among individual patients.

\begin{abstract}
Abbreviations:
AGREE, Appraisal of guidelines for research and evaluation; ALT, alanine aminotransferase; anti-HCV, antibody to hepatitis C; APRI, aminotransferaseto-platelet ratio index; AST, aspartate aminotransferase; ASV, asunaprevir; AUC, area under the curve; /r, /ritonavir; CTP, Child-Pugh Turcotte; eGFR, estimated glomerular filtration rate; DAA, direct-acting antivirals; DCV, daclatasvir; DDI, drug-drug interactions; EBR/GZR, elbasvir/grazoprevir; ESRD, end-stage renal disease; ETR, end-of treatment response; FIB-4, fibrosis-4 score; GFR, glomerular filtration rate; G/P, glecaprevir/pibrentasvir; GRADE, grading of recommendations, assessment, development and evaluation; HBV, hepatitis B virus; HCC, hepatocellular carcinoma; HCV, hepatitis C virus; HIV, human immunodeficiency virus; IDU, injection drug user; KASL, Korean Association for the Study of the Liver; LC, liver cirrhosis; LED/SOF, ledipasvir/sofosbuvir; MELD, model for end-stage liver disease; MSM, man who have sex with man; NNRTI, non-nucleoside reverse transcriptase inhibitors; NRTI, nucleoside reverse transcriptase inhibitors; NS, nonstructural protein; NS3, nonstructural protein 3; NS5A, nonstructural protein 5A; OATP, organic anion-transporting polypeptide; Opr+D, ombitasvir/paritaprevir/ritonavir and dasabuvir; PCR, polymerase chain reaction; PegIFN, peginterferon; $\mathrm{P}$-gp, P-glycoprotein; $\mathrm{Pl}$, protease inhibitor; $P R$, peg interferon-alpha + ribavirin therapy; PWID, people who inject drugs; $R^{*}$, ribavirin started from $600 \mathrm{mg} / \mathrm{d}$; R, weight-based ribavirin; RASs, resistanceassociated substitutions; SOF, sofosbuvir; SOF/VEL, sofosbuvir/velpatasvir; SOF/ VEL/VOX, sofosbuvir/velpatasvir/voxilaprevir; SVR, sustained virologic response rate; TDF, tenofovir disoproxil fumarate
\end{abstract}

Corresponding author: The Korean Association for the Study of the Liver (KASL) (Committee Chair: Jong Eun Yeon)

Room A1210 MapoTrapalace, 53 Mapo-daero, Mapo-gu, Seoul 04158, Korea

Tel: +82-2-703-0051, Fax: +82-2-703-0071

E-mail: kasl@kams.or.kr

* Clinical Practice Guidelines Committee of KASL for the Management of Hepatitis C: Treatment of chronic hepatitis C Jong Eun Yeon (Committee Chair, Korea University College of Medicine), In Hee Kim (Chonbuk National University Medical School), Jung II Lee (Yonsei University College of Medicine), Kyung-Ah Kim (Inje University College of Medicine), Geum-Youn Gwak (Sungkyunkwan University School of Medicine), Ji Hoon Kim (Korea University College of Medicine), Kang Mo Kim (University of Ulsan College of Medicine), Jeong Won Jang (College of Medicine, The Catholic University of Korea), Do Young Kim (Yonsei University College of Medicine), and Ki Tae Yoon (Pusan National University School of Medicine) 


\section{Target populations}

The target groups for these guidelines are newly or previously diagnosed patients with hepatitis C virus infection (HCV), including patients with chronic hepatitis $\mathrm{C}$ with cirrhosis, hepatitis $\mathrm{C}$ patients with chronic kidney disease, and those co-infected with human immunodeficiency virus (HIV) or hepatitis B virus (HBV).

\section{Intended users}

These guidelines are intended to provide useful information and guidance to physicians and healthcare providers involved in the diagnosis and treatment of hepatitis $C$, along with resident physicians, practitioners, and trainers.

\section{Development, funding, and revision process}

The Clinical Practice Guidelines Committee for the Management of Hepatitis C, comprising 10 hepatologists, was organized according to a proposal by and the approval of the KASL Board of Executives. Funding for the revision was provided by KASL. Each committee member collected and analyzed the source data in his or her own field, and the members then wrote the manuscript together.

\section{Literature review for evidence collection}

The committee systematically collected and reviewed the international and domestic literature published before September 2017 in PubMed, MEDLINE, KoreaMed, and other databases. The keywords used were 'hepatitis C virus', 'hepatitis C', 'liver cirrhosis', 'liver cancer', and other specific related keywords.

\section{Level of evidence and grades of recommendations}

The quality of evidence is classified using the Grading of Recommendations, Assessment, Development, and Evaluation (GRADE) system (Table 1).' Based on the types of study, randomized controlled studies were approached as providing a high level of evidence, whereas observational studies were approached as providing a low level of evidence. Then, the levels of evidence were adjusted by accounting for the factors that influence study quality. The levels of evidence are defined as follows: A, the highest level of evidence with the smallest possibility of changes in the conclusion; B, a moderate risk of potential changes; and C, the lowest level of evidence with the greatest possibility of changes.

The strength of each recommendation is also classified according to the GRADE system. Each study was classified as a strong recommendation (1) or weak recommendation (2) based on the quality of evidence, the balance between the desirable and undesirable effects of an intervention, and socioeconomic aspects, including cost and availability. A strong recommendation indicates that the intervention could be applied for most patients with a strong certainty that desirable effects are highly possible with high-quality evidence, presumed patient-important outcomes, cost-effectiveness, preference, and compliance. A weak recommendation indicates a suggestion made with less certainty but that still could be considered favorable for many patients based

Table 1. Grading of recommendations, assessment, development, and evaluation (GRADE)

\begin{tabular}{|c|c|}
\hline & Criteria \\
\hline \multicolumn{2}{|c|}{ Quality of evidence } \\
\hline $\operatorname{High}(A)$ & Further research is very unlikely to change our confidence in the estimate of effect. \\
\hline Moderate (B) & $\begin{array}{l}\text { Further research is likely to have an important impact on our confidence in the estimate of effect and may } \\
\text { change the estimate. }\end{array}$ \\
\hline $\operatorname{Low}(\mathrm{C})$ & $\begin{array}{l}\text { Further research is very likely to have an important impact on our confidence in the estimate of effect and is } \\
\text { likely to change the estimate. Any change of estimate is uncertain. }\end{array}$ \\
\hline \multicolumn{2}{|c|}{ Strength of recommendation } \\
\hline Strong (1) & $\begin{array}{l}\text { Factors influencing the strength of the recommendation include the quality of the evidence, presumed } \\
\text { patient-important outcomes, and cost. }\end{array}$ \\
\hline Weak (2) & $\begin{array}{l}\text { Variability in preference and values, or more uncertainty. Recommnedation is made with less certainty, } \\
\text { higher cost or resource consumption. }\end{array}$ \\
\hline
\end{tabular}

Of the quality of evidence, this guideline excluded "very low quality (D)" in our guideline for convenience, which was originally included in the GRADE system. 
The Korean Association for the Study of the Liver (KASL) 2017 KASL clinical practice guidelines: Management of chronic hepatitis C

on the level of evidence and the cost or preferences of patients or medical practitioners.

\section{List of key questions}

The revision committee considered the following clinical questions as the key components to be covered in these guidelines.

1. What are the characteristics and indications of new kinds of DAAs?

2. What are the recent updates in drug-drug interactions?

3. What is the definition, detection method, and clinical application of resistance associated substitutions (RASs)?

4. How should patients with genotype 1 chronic hepatitis and compensated cirrhosis be treated?

5. How should patients with genotype 2 chronic hepatitis and compensated cirrhosis be treated?

6. How should patients with genotype 3 chronic hepatitis and compensated cirrhosis be treated?

7. How should patients with genotype 4 chronic hepatitis and compensated cirrhosis be treated?

8. How should patients with genotype 5 or 6 chronic hepatitis and compensated cirrhosis be treated?

9. How should patients with decompensated cirrhosis be treated?

10. How should patients who underwent liver or extrahepatic organ transplantation be treated?

11. How should patients with special conditions (people who inject drug, chronic kidney disease, coinfection with HIV or HBV, hemophilia or thalassemia, immunosuppressive therapy or cytotoxic chemotherapy, and pediatric patients) be treated?

12. How should patients who failed on previous direct antiviral agents be treated?

\section{Review of the manuscript and approval process}

Each manuscript written by committee members was reviewed, agreed, and approved through meetings of the committee. The quality of the manuscript was evaluated based on the standards suggested by Appraisal of Guidelines for Research and Evaluation II (AGREE II), along with the academic integrity of the contents. The guidelines were reviewed and revised based on counsel from an infection specialist, a meeting of an external review board of 7 KASL members, opinions at a public hearing, and a symposium open to all KASL members. The final manuscript was approved by the KASL Board of Executives.

\section{Release of the guidelines and plan for updates}

The Korean version of the KASL Clinical Practice Guidelines for the Management of Hepatitis C: Treatment of Chronic Hepatitis C was released in November 2017 at a KASL meeting and published in November 2017 on the KASL website (http://www.kasl.org). Future plans for revision will be made when the accumulation of research on the management of hepatitis $C$ makes revision necessary for the promotion of health in South Korea. In addition, the use of new DAAs will be allowed in South Korea in the near future, which might warrant updates or revisions to the guidelines.

\section{DIRECT ACTING ANTIVIRALS (DAAS)}

Recently, the paradigm for treating hepatitis C changed rapidly as newly developed oral antiviral agents, DAAs, became available. DAAs show antiviral effects by directly acting on the life cycle of the hepatitis C virus (HCV). DAAs are classified into HCV nonstructural protein (NS) 3/4A protease inhibitors, NS5A inhibitors, and NS5B polymerase inhibitors, depending on their site of action. NS3/4A protease inhibitors are first-generation DAAs that block the polyprotein processing essential for HCV replication. Following the first-generation protease inhibitors (boceprevir and telaprevir), simeprevir, asunaprevir, paritaprevir, grazoprevir, voxilaprevir, and glecaprevir were developed. ${ }^{2}$ NS5A inhibitors affect $\mathrm{HCV}$ replication and assembly, showing a synergistic effect in combination with other DAAs. They include daclatasvir, ledipasvir, ombitasvir, elbasvir, velpatasvir, and pibrentasvir. NS5B polymerase inhibitors include sofosbuvir and dasabuvir.

As of 2017, the DAAs sofosbuvir, ledipasvir/sofosbuvir, daclatasvir, asunaprevir, ombitasvir/paritaprevir/ritonavir, dasabuvir, elbasvir/grazoprevir were approved in Korea (Table 2). In the United States and Europe, sofosbuvir/velpatasvir was approved in 2016, and sofosbuvir/velpatasvir/voxilaprevir and glecaprevir/pibrentasvir were approved in 2017.

Given an understanding of the basic characteristics, doses, and posology of each DAA, the selection and use of appropriate drugs should consider hepatic and renal function. DAA regimens can interact with other medications used by patients. Prior to starting treatment, patients should be evaluated for potential drug-drug interactions with selected DAAs (Table 3, 4). A comprehensive list of drug-drug interactions is available at several websites, such as www.hep-druginteractions.org ${ }_{2}$ and in the prescribing information for each drug. ${ }^{2-8}$ 


\section{[Recommendations]}

1. Clinicians should understand the characteristics of each DAA and choose the most appropriate drug for each patient, with consideration of hepatic and renal function (A1).

2. The potential for drug-drug interactions must be considered before and during treatment with DAAs. Full prescribing information must be consulted prior to the use of DAAs because of the potential for drug-drug interactions (A1).

\section{Sofosbuvir}

Sofosbuvir is an HCV NS5B polymerase inhibitor.

\section{Dosage and administration}

Sofosbuvir should be administered orally at the dose of $400 \mathrm{mg}$ (one tablet) once daily, with or without food.

\section{Pharmacokinetics}

Sofosbuvir is metabolized in the liver by the cathepsin A, carboxylesterase 1, histidine triad nucleotide-binding protein 1, and pyrimidine nucleotide biosynthesis pathway. GS-331007 is a major metabolite of sofosbuvir, of which approximately $80 \%$ is excreted in urine, and $15 \%$ is excreted in feces. No dosage adjustment of sofosbuvir is required for patients with hepatic impairment. Compared to subjects with normal renal function (eGFR $>80 \mathrm{~mL} / \mathrm{min}$ ), the area under the curve (AUC) of sofosbuvir and GS-331007 were 1.7-fold and 4.5-fold higher, respectively, in patients with severe renal impairment (eGFR $<30 \mathrm{~mL} / \mathrm{min}$ ) and that of GS331007 was increased up to 20 -fold in patients with end-stage renal disease (ESRD). Thus, no dosage adjustment of sofosbuvir is required for patients with mild or moderate renal impairment (eGFR 30-80 $\mathrm{mL} / \mathrm{min}$ ). Sofosbuvir is not recommended for use in patients with severe renal impairment (eGFR $<30 \mathrm{~mL} / \mathrm{min}$ ) or ESRD requiring dialysis.

\section{Drug-drug interactions}

Sofosbuvir is a substrate of the drug transporter P-glycoprotein (P-gp) and the breast cancer resistance protein (BCRP). Strong P-gp inducers, including anticonvulsants (carbamazepine, oxcarbazepine, phenobarbital, phenytoin), antituberculosis drugs (rifampin, rifabutin, rifapentine), and the herbal product St. John's wort, could decrease the plasma concentration of sofosbuvir, causing reduced therapeutic efficacy. Thus, concomitant use of those substances with sofosbuvir is not recommended. In addition, because serious symptomatic bradycardia can occur, co-administration of amiodarone with sofosbuvir is contraindicated.

Table 2. Direct acting antivirals and ribavirin for hepatitis $C$ virus treatment

\begin{tabular}{|c|c|c|c|}
\hline Product & Brand name & Presentation & Posology \\
\hline Sofosbuvir* & SOVALDI ${ }^{\oplus}$ & Sofosbuvir 400 mg (1 tablet) & One tablet once a day with or without food \\
\hline Ledipasvir/sofosbuvir* & HARVONI ${ }^{\otimes}$ & $\begin{array}{l}\text { Ledipasvir } 90 \text { mg/sofosbuvir } 400 \mathrm{mg} \\
\text { (1 tablet) }\end{array}$ & One tablet once a day with or without food \\
\hline Daclatasvir* & DAKLINZA $^{\oplus}$ & Daclatasvir 60 or 30 mg (1 tablet) & One tablet once a day with or without food \\
\hline Asunaprevir* & SUNVEPRA ${ }^{\oplus}$ & Asunaprevir 100 mg (1 capsule) & One capsule twice a day with or without food \\
\hline $\begin{array}{l}\text { Ombitasvir/paritaprevir/ } \\
\text { ritonavir* }^{*}\end{array}$ & VIEKIRAX ${ }^{\circledast}$ & $\begin{array}{l}\text { Ombitasvir } 12.5 \text { mg/paritaprevir } 75 \text { mg/ } \\
\text { ritonavir } 50 \text { mg (1 tablet) }\end{array}$ & Two tablets once a day with food \\
\hline Dasabuvir* & EXVIERA ${ }^{\oplus}$ & Dasabuvir 250 mg (1 tablet) & One tablet twice a day with food \\
\hline Elbasvir/grazoprevir* & ZEPATIER ${ }^{\oplus}$ & $\begin{array}{l}\text { Elbasvir } 50 \text { mg/ } \\
\text { grazoprevir } 100 \text { mg (1 tablet) }\end{array}$ & One tablet once a day with or without food \\
\hline Glecaprevir/pibrentasvir* & MAVYRET ${ }^{\oplus}$ & $\begin{array}{c}\text { Glecaprevir } 100 \text { mg/pibrentasvir } 40 \text { mg } \\
\text { (1 tablet) }\end{array}$ & Three tablets once a day with food \\
\hline Sofosbuvir/velpatasvir & EPCLUSA $^{\oplus}$ & $\begin{array}{l}\text { Sofosbuvir } 400 \text { mg/ } \\
\text { velpatasvir } 100 \text { mg (1 tablet) }\end{array}$ & One tablet once a day with or without food \\
\hline $\begin{array}{l}\text { Sofosbuvir/velpatasvir/ } \\
\text { voxilaprevir }\end{array}$ & VOSEVI $\left.\right|^{\oplus}$ & $\begin{array}{c}\text { Sofosbuvir } 400 \text { mg/ } \\
\text { velpatasvir } 100 \text { mg/ } \\
\text { voxilaprevir } 100 \text { mg (1 tablet) }\end{array}$ & One tablet once a day with food \\
\hline Ribavirin* & VIRAMID $^{\oplus}, \mathrm{RIBAVIRIN}^{\oplus}$ & Ribavirin 200 mg (1 capsule) & $\begin{array}{l}\text { If body weight }<75 \mathrm{~kg}, 1,000 \mathrm{mg} / \text { day; } \\
\text { If body weight } \geq 75 \mathrm{~kg}, 1,200 \mathrm{mg} / \text { day }\end{array}$ \\
\hline
\end{tabular}

${ }^{*}$ Approved by the Korean Ministry of Food and Drug Safety. 
The Korean Association for the Study of the Liver (KASL) 2017 KASL clinical practice guidelines: Management of chronic hepatitis C

Table 3. Drug interactions between hepatitis $C$ virus DAAs and selected cardiovascular and metabolic drugs

\begin{tabular}{|c|c|c|c|c|c|c|c|c|c|}
\hline Co-medications & SOF & LDV/SOF & DCV & ASV & OPr-D & EBR/GZR & $\mathrm{G} / \mathrm{P}$ & SOF/VEL & SOF/VEL/VOX \\
\hline \multicolumn{10}{|l|}{ Angiotensin inhibitors } \\
\hline Aliskiren & 0 & $\Delta$ & $\Delta$ & NA & $x$ & 0 & $x$ & O & $\Delta$ \\
\hline Enalapril & 0 & 0 & 0 & 0 & $\Delta$ & 0 & $\Delta$ & 0 & $\Delta$ \\
\hline Candesartan & 0 & 0 & 0 & NA & 0 & $\Delta$ & $\Delta$ & 0 & $\Delta$ \\
\hline Losartan & 0 & 0 & 0 & 0 & 0 & 0 & 0 & 0 & 0 \\
\hline Telmisartan & 0 & 0 & 0 & NA & $\Delta$ & 0 & $\Delta$ & O & $\Delta$ \\
\hline \multicolumn{10}{|l|}{ Antiarrhythmics } \\
\hline Amiodarone & $x$ & $x$ & $x$ & 0 & $x$ & $\Delta$ & $\Delta$ & $x$ & $x$ \\
\hline Digoxin & 0 & $\Delta$ & $\Delta$ & $\Delta$ & $\Delta$ & 0 & $\Delta$ & $\Delta$ & $\Delta$ \\
\hline Dronedarone & $x$ & $x$ & $x$ & NA & $x$ & $\Delta$ & $\Delta$ & $x$ & $x$ \\
\hline Flecainide & 0 & 0 & 0 & $x$ & $\Delta$ & 0 & 0 & 0 & 0 \\
\hline \multicolumn{10}{|c|}{ Antiplatelets or anticoagulants } \\
\hline Aspirin & 0 & 0 & 0 & NA & 0 & 0 & 0 & 0 & 0 \\
\hline Clopidogrel & 0 & 0 & 0 & NA & $\Delta$ & 0 & 0 & 0 & 0 \\
\hline Dabigatran & 0 & $\Delta$ & $\Delta$ & $\Delta$ & $\Delta$ & $\Delta$ & $x$ & $\Delta$ & $x$ \\
\hline Ticagrelor & 0 & $\Delta$ & 0 & NA & $x$ & $\Delta$ & $\Delta$ & $\Delta$ & $\Delta$ \\
\hline Warfarin & 0 & 0 & O & O & $\Delta$ & O & $\Delta$ & O & $\Delta$ \\
\hline \multicolumn{10}{|l|}{ Beta blockers } \\
\hline Atenolol & 0 & 0 & 0 & NA & 0 & 0 & 0 & 0 & 0 \\
\hline Carvedilol & 0 & $\Delta$ & $\Delta$ & NA & $\Delta$ & 0 & $\Delta$ & $\Delta$ & $\Delta$ \\
\hline Propranolol & 0 & 0 & 0 & NA & $\Delta$ & 0 & 0 & 0 & 0 \\
\hline \multicolumn{10}{|c|}{ Calcium channel blockers } \\
\hline Amlodipine & 0 & $\Delta$ & $\Delta$ & NA & $\Delta$ & $\Delta$ & 0 & $\Delta$ & 0 \\
\hline Diltiazem & 0 & $\Delta$ & $\Delta$ & $x$ & $\Delta$ & 0 & $\Delta$ & $\Delta$ & $\Delta$ \\
\hline Nifedipine & 0 & 0 & $\Delta$ & NA & $\Delta$ & O & 0 & O & O \\
\hline \multicolumn{10}{|l|}{ Diuretics } \\
\hline Furosemide & 0 & 0 & 0 & NA & $\Delta$ & 0 & 0 & 0 & 0 \\
\hline Hydrochlorothiazide & 0 & 0 & 0 & NA & 0 & 0 & 0 & 0 & 0 \\
\hline \multicolumn{10}{|l|}{ Glucose lowering drugs } \\
\hline Metformin & 0 & 0 & 0 & NA & 0 & 0 & 0 & 0 & 0 \\
\hline Gliclazide & 0 & 0 & 0 & NA & $\Delta$ & 0 & 0 & 0 & 0 \\
\hline Glimepiride & 0 & 0 & 0 & NA & 0 & 0 & 0 & 0 & 0 \\
\hline Sitagliptin & 0 & 0 & 0 & NA & 0 & 0 & 0 & 0 & 0 \\
\hline \multicolumn{10}{|l|}{ Lipid lowering drugs } \\
\hline Atorvastatin & 0 & $\Delta$ & $\Delta$ & $\Delta$ & $x$ & $\Delta$ & $x$ & $\Delta$ & $x$ \\
\hline Bezafibrate & 0 & 0 & 0 & NA & 0 & 0 & 0 & 0 & 0 \\
\hline Ezetimibe & 0 & 0 & 0 & NA & $\Delta$ & 0 & $\Delta$ & 0 & $\Delta$ \\
\hline Fenofibrate & 0 & 0 & 0 & NA & 0 & 0 & $\Delta$ & 0 & 0 \\
\hline Fluvastatin & 0 & $\Delta$ & $\Delta$ & $\Delta$ & $\Delta$ & $\Delta$ & $\Delta$ & $\Delta$ & $x$ \\
\hline Gemfibrozil & 0 & 0 & 0 & $x$ & $x$ & $\Delta$ & $\Delta$ & 0 & 0 \\
\hline Lovastatin & 0 & $\Delta$ & $\Delta$ & NA & $x$ & $\Delta$ & $x$ & $\Delta$ & $x$ \\
\hline Pitavastatin & 0 & $\Delta$ & $\Delta$ & $\Delta$ & $\Delta$ & 0 & $\Delta$ & $\Delta$ & $X$ \\
\hline Pravastatin & 0 & $\Delta$ & $\Delta$ & $\Delta$ & $\Delta$ & 0 & $\Delta$ & 0 & $\Delta$ \\
\hline Rosuvastatin & 0 & $x$ & $\Delta$ & $\Delta$ & $\Delta$ & $\Delta$ & $\Delta$ & $\Delta$ & $x$ \\
\hline Simvastatin & 0 & $\Delta$ & $\Delta$ & $\Delta$ & $x$ & $\Delta$ & $x$ & $\Delta$ & $x$ \\
\hline
\end{tabular}

DAA, direct acting antiviral; SOF, sofosbuvir; LDV, ledipasvir; DCV, daclatasvir; ASV, asunaprevir; OPr-D, ombitasvir/paritaprevir/ritonavir plus dasabuvir; EBR/GZR, elbasvir/grazoprevir; G/P, glecaprevir/pibrentasvir; VEL, velpatasvir; VOX, voxilaprevir; 0 , no clinical significant interaction expected; $\triangle$, potential interaction that might require dose adjustment, altered timing of administration, or additional monitoring; NA, not available; $X$, these drugs should not be coadministered. 
Table 4. Drug interactions between hepatitis C virus DAAs and other selected co-medications

\begin{tabular}{|c|c|c|c|c|c|c|c|c|c|}
\hline Co-medications & SOF & LDV/SOF & DCV & ASV & OPr-D & EBR/GZR & $\mathrm{G} / \mathrm{P}$ & SOF/VEL & SOF/VEL/VOX \\
\hline \multicolumn{10}{|l|}{ Anticonvulsants } \\
\hline $\begin{array}{l}\text { Carbamazepine, oxcarbazepine, } \\
\text { phenobarbital, phenytoin }\end{array}$ & $x$ & $x$ & $x$ & $x$ & $x$ & $x$ & $x$ & $x$ & $x$ \\
\hline \multicolumn{10}{|l|}{ Antidepressants } \\
\hline Amitriptyline & 0 & 0 & 0 & $\Delta$ & 0 & 0 & 0 & 0 & 0 \\
\hline Citalopram, escitalopram & 0 & 0 & 0 & 0 & 0 & 0 & 0 & 0 & 0 \\
\hline Duloxetine & 0 & 0 & 0 & NA & 0 & 0 & 0 & 0 & 0 \\
\hline Fluoxetine & 0 & 0 & 0 & NA & 0 & 0 & 0 & 0 & 0 \\
\hline Sertraline & 0 & 0 & 0 & 0 & $\Delta$ & 0 & 0 & 0 & 0 \\
\hline Trazodone & 0 & 0 & 0 & NA & $\Delta$ & 0 & 0 & 0 & 0 \\
\hline Venlafaxine & 0 & 0 & 0 & NA & $\Delta$ & 0 & 0 & 0 & 0 \\
\hline \multicolumn{10}{|l|}{ Antifungals } \\
\hline Fluconazole & 0 & 0 & 0 & $x$ & 0 & 0 & 0 & 0 & 0 \\
\hline Itraconazole & 0 & 0 & $\Delta$ & $x$ & $x$ & 0 & 0 & 0 & 0 \\
\hline Ketoconazole & 0 & 0 & $\Delta$ & $x$ & $x$ & $\Delta$ & $\Delta$ & 0 & 0 \\
\hline Posaconazole & 0 & 0 & $\Delta$ & $x$ & $x$ & 0 & $\Delta$ & 0 & 0 \\
\hline Voriconazole & 0 & 0 & $\Delta$ & $x$ & $x$ & 0 & 0 & 0 & 0 \\
\hline \multicolumn{10}{|l|}{ Antipsychotics } \\
\hline Amisulpride & 0 & 0 & 0 & NA & 0 & 0 & 0 & 0 & 0 \\
\hline Aripiprazole & 0 & 0 & 0 & $\Delta$ & $\Delta$ & $\Delta$ & $\Delta$ & 0 & 0 \\
\hline Chlorpromazine & 0 & 0 & 0 & NA & $\Delta$ & 0 & 0 & 0 & 0 \\
\hline Clozapine & 0 & 0 & 0 & $\Delta$ & $\Delta$ & 0 & $\Delta$ & 0 & 0 \\
\hline Haloperidol & 0 & 0 & 0 & NA & $\Delta$ & 0 & 0 & 0 & 0 \\
\hline Olanzapine & 0 & 0 & 0 & NA & $\Delta$ & 0 & 0 & 0 & 0 \\
\hline Paliperidone & 0 & $\Delta$ & $\Delta$ & NA & 0 & 0 & $\Delta$ & 0 & $\Delta$ \\
\hline Quetiapine & 0 & 0 & 0 & NA & $x$ & $\Delta$ & $\Delta$ & 0 & 0 \\
\hline Risperidone & 0 & 0 & 0 & NA & $\Delta$ & 0 & 0 & 0 & 0 \\
\hline \multicolumn{10}{|l|}{ Antituberculosis drugs } \\
\hline Rifampin & $x$ & $x$ & $x$ & $x$ & $x$ & $x$ & $x$ & $x$ & $x$ \\
\hline \multicolumn{10}{|l|}{ Gastric acid lowering drugs } \\
\hline Famotidine & 0 & $\Delta$ & 0 & 0 & 0 & 0 & $\Delta$ & $\Delta$ & $\Delta$ \\
\hline Omeprazole & 0 & $\Delta$ & 0 & 0 & $\Delta$ & 0 & $\Delta$ & $\Delta$ & $\Delta$ \\
\hline \multicolumn{10}{|l|}{ Herbal products } \\
\hline St. John's wort & $x$ & $x$ & $x$ & $x$ & $x$ & $x$ & $x$ & $x$ & $x$ \\
\hline \multicolumn{10}{|l|}{ Immunosuppressive drugs } \\
\hline Azathioprine & 0 & 0 & 0 & NA & 0 & 0 & 0 & 0 & 0 \\
\hline Cyclosporine & 0 & 0 & 0 & $x$ & $\Delta$ & $x$ & $\Delta$ & 0 & $x$ \\
\hline Etanercept & $\Delta$ & $\Delta$ & $\Delta$ & NA & $\Delta$ & $\Delta$ & $\Delta$ & $\Delta$ & $\Delta$ \\
\hline Everolimus & 0 & $\Delta$ & $\Delta$ & NA & $\Delta$ & $\Delta$ & $\Delta$ & $\Delta$ & $\Delta$ \\
\hline Mycophenolate & 0 & 0 & 0 & NA & $\Delta$ & 0 & 0 & 0 & 0 \\
\hline Sirolimus & 0 & 0 & 0 & $x$ & $\Delta$ & $\Delta$ & $\Delta$ & 0 & $\Delta$ \\
\hline
\end{tabular}


The Korean Association for the Study of the Liver (KASL) 2017 KASL clinical practice guidelines: Management of chronic hepatitis C

Table 4. Continued

\begin{tabular}{|c|c|c|c|c|c|c|c|c|c|}
\hline Co-medications & SOF & LDV/SOF & DCV & ASV & OPr-D & EBR/GZR & $\mathrm{G} / \mathrm{P}$ & SOF/VEL & SOF/VEL/VOX \\
\hline Tacrolimus & 0 & 0 & 0 & NA & $\Delta$ & $\Delta$ & $\Delta$ & 0 & $\Delta$ \\
\hline \multicolumn{10}{|l|}{ Macrolides } \\
\hline Azithromycin & 0 & 0 & 0 & 0 & 0 & 0 & 0 & 0 & 0 \\
\hline Clarithromycin & 0 & 0 & $\Delta$ & $x$ & $x$ & 0 & $\Delta$ & 0 & $\Delta$ \\
\hline Erythromycin & 0 & 0 & $\Delta$ & $x$ & $\Delta$ & 0 & $\Delta$ & 0 & $\Delta$ \\
\hline Telithromycin & 0 & 0 & $\Delta$ & NA & $x$ & $\Delta$ & $\Delta$ & 0 & $\Delta$ \\
\hline \multicolumn{10}{|l|}{ Opioids } \\
\hline Buprenorphine & 0 & $\Delta$ & 0 & 0 & $\Delta$ & 0 & 0 & 0 & $\Delta$ \\
\hline Methadone & 0 & 0 & 0 & 0 & 0 & 0 & 0 & 0 & 0 \\
\hline \multicolumn{10}{|l|}{ Sedatives } \\
\hline Midazolam (oral) & 0 & $\Delta$ & 0 & $\Delta$ & $x$ & $\Delta$ & 0 & 0 & 0 \\
\hline Midazolam (parenteral) & 0 & $\Delta$ & 0 & NA & $\Delta$ & $\Delta$ & 0 & 0 & 0 \\
\hline Triazolam & 0 & 0 & 0 & NA & $x$ & 0 & 0 & 0 & 0 \\
\hline \multicolumn{10}{|l|}{ Systemic steroids } \\
\hline Dexamethasone & 0 & 0 & $x$ & $x$ & $\Delta$ & $\Delta$ & $\Delta$ & 0 & $\Delta$ \\
\hline Prednisone & 0 & 0 & 0 & NA & $\Delta$ & 0 & 0 & 0 & 0 \\
\hline \multicolumn{10}{|l|}{ Others } \\
\hline Bosentan & 0 & 0 & $\Delta$ & $x$ & $x$ & $x$ & $x$ & $x$ & $x$ \\
\hline Colchicine & 0 & $\Delta$ & $\Delta$ & NA & $\Delta$ & $\Delta$ & $\Delta$ & $\Delta$ & $\Delta$ \\
\hline Ergotamine & 0 & $\Delta$ & 0 & NA & $x$ & $\Delta$ & $\Delta$ & 0 & 0 \\
\hline Ethinylestradiol & 0 & 0 & 0 & $\Delta$ & $x$ & 0 & $x$ & 0 & $x$ \\
\hline Sildenafil & 0 & 0 & 0 & 0 & $\Delta$ & 0 & 0 & 0 & 0 \\
\hline
\end{tabular}

DAA, direct acting antiviral; SOF, sofosbuvir; LDV, ledipasvir; DCV, daclatasvir; ASV, asunaprevir; OPr-D, ombitasvir/paritaprevir/ritonavir plus dasabuvir; EBR/ GZR, elbasvir/grazoprevir; G/P, glecaprevir/pibrentasvir; VEL, velpatasvir; VOX, voxilaprevir; $X$, these drugs should not be co-administered; 0 , no clinical significant interaction expected; $\triangle$, potential interaction that might require dose adjustment, altered timing of administration, or additional monitoring; NA, not available.

\section{Adverse reactions and safety}

The most common adverse reactions observed with sofosbuvir in combination with ribavirin were fatigue and headache, insomnia, pruritus, and anemia.

\section{Ledipasvir/sofosbuvir}

Ledipasvir is an HCV NS5A inhibitor, and sofosbuvir is an HCV NS5B polymerase inhibitor.

\section{Dosage and administration}

Ledipasvir/sofosbuvir is a fixed-dose combination of ledipasvir $(90 \mathrm{mg}$ ) and sofosbuvir (400 $\mathrm{mg}$ ) in a single tablet and should be administered at the dose of one tablet orally once a day, with or without food.

\section{Pharmacokinetics}

Ledipasvir is subject to slow oxidative metabolism via a still unknown mechanism. Ledipasvir is mainly excreted in feces, whereas, sofosbuvir is extensively excreted via the kidneys. No dosage adjustment of ledipasvir/sofosbuvir is required for patients with hepatic impairment due to minimal effects in the plasma concentration of the drug. No dose adjustment is required for patients with mild or moderate renal impairment (eGFR $30-80 \mathrm{~mL} / \mathrm{min}$ ), but it is not recommended for use in patients with severe renal impairment (eGFR $<30 \mathrm{~mL} / \mathrm{min}$ ) or ESRD because its safety is not established in that population.

\section{Drug-drug interactions}

Ledipasvir and sofosbuvir are substrates of the drug transporters P-gp and BCRP. Strong P-gp inducers, such as antituberculosis drugs (rifampin, rifapentine, rifabutin), anticonvulsants (carbam- 
azepine, oxcarbazepine, phenobarbital, phenytoin), and the herbal product St. John's wort, could decrease the plasma concentration of ledipasvir/sofosbuvir and cause reduced therapeutic efficacy. Thus, concomitant use of those substances with ledipasvir/sofosbuvir is not recommended. Ledipasvir is an inhibitor of drug transporters such as P-gp, BCRP, and organic anion transporting polypeptide (OATP). Co-administration of ledipasvir/sofosbuvir with P-gp substrates (digoxin, dabigatran) could increase the concentration of each drug, requiring careful monitoring for therapeutic concentration or side effects. Co-administration with rosuvastatin is contraindicated because ledipasvir inhibits OATP, leading to a significant increase in the concentration of rosuvastatin. In addition, caution about adverse reactions is warranted if other HMG$\mathrm{Co} A$ reductase inhibitors (atorvastatin, fluvastatin, lovastatin, pitavastatin, pravastatin, simvastatin) are used. Co-administration of amiodarone (and possibly dronedarone) with ledipasvir/sofosbuvir is contraindicated due to serious risk of symptomatic bradycardia. Because the solubility of ledipasvir decreases with increasing $\mathrm{pH}$, drugs that increase gastric $\mathrm{pH}$ are expected to decrease the concentration of ledipasvir. It is therefore recommended to separate antacids and ledipasvir/sofosbuvir by 4 hours. H2-receptor antagonists may be administered simultaneously with or 12 hours apart from ledipasvir/sofosbuvir at a dose that does not exceed doses comparable to famotidine $40 \mathrm{mg}$ twice daily. Protonpump inhibitors can be administered at doses comparable to omeprazole $20 \mathrm{mg}$ or lower, but at higher doses, they might decrease the efficacy of ledipasvir/sofosbuvir. Because ledipasvir/sofosbuvir increases the tenofovir concentration when a pharmacokinetic enhancer (ritonavir or cobicistat) is included in an antiretroviral regimen, combinations such as atazanavir/ritonavir, darunavir/ritonavir, lopinavir/ritonavir, elvitegravir/cobicistat, atazanavir/cobicistat, or darunavir/cobicistat in combination with tenofovir disoproxil fumarate/emtricitabine should be used with caution and frequent renal monitoring.

\section{Adverse reactions and safety}

The most common adverse reactions observed with ledipasvir/ sofosbuvir were fatigue, headache, diarrhea, and insomnia.

\section{Daclatasvir}

Daclatasvir is an HCV NS5A inhibitor.

\section{Dosage and administration}

Daclatasvir is orally administered at the dose of $60 \mathrm{mg}$ (one tablet) once daily with or without food. Dose modification of daclatasvir is needed if a CYP3A inhibitor or inducer is co-administered. If a strong CYP3A inhibitor is co-administered, the dose of daclatasvir should be reduced to one $30 \mathrm{mg}$ tablet once daily. If a moderate CYP3A inducer is co-administered, the dose of daclatasvir should be increased to one $90 \mathrm{mg}$ tablet once daily. Co-administration with a strong CYP3A inducer is contraindicated.

\section{Pharmacokinetics}

Daclatasvir is metabolized by CYP3A. Approximately $90 \%$ of daclatasvir is eliminated in feces, with less than $10 \%$ excreted in urine. Hepatic impairment does not have a clinically significant effect on the free drug concentration of daclatasvir. Thus, no dosage adjustment of daclatasvir is required for patients with any degree of hepatic or renal impairment.

\section{Drug-drug interactions}

Co-administration of daclatasvir with substances that are moderate or strong inducers or inhibitors of CYP3A4 is not recommended because it can significantly alter the exposure to daclatasvir. Strong CYP3A inducers, including anticonvulsants (carbamazepine, oxcarbazepine, phenobarbital, phenytoin), the herbal product St. John's wort, and the antituberculosis drug rifampin, are contraindicated. Strong CYP3A inhibitors, including antiretrovirals (atazanavir, ritonavir, indinavir, nelfinavir, saquinavir, atazanavir/cobicistat, elvitegravir/cobicistat/emtricitabine/tenofovir disoproxil fumarate), antifungals (itraconazole, ketoconazole, posaconazole, voriconazole), macrolides (clarithromycin, telithromycin), the antidepressant nefazodone, and calcium channel blockers (diltiazem, verapamil), can increase the plasma concentration of daclatasvir. Thus, the dose of daclatasvir should be decreased, or co-administration is not recommended. Moderate CYP3A inducers, including antibiotics (nafcillin, rifapentine), the endothelin receptor antagonist bosentan, the systemic steroid dexamethasone, antiretrovirals (efavirenz, etravirine, nevirapine), and the stimulant drug modafinil, can decrease the plasma concentration of daclatasvir. Thus, the dose of daclatasvir should be increased, or co-administration is not recommended. Daclatasvir is an inhibitor of P-gp, OATP1B1 and 1B3, and BCRP. Therefore, co-administration with the antiarrhythmic digoxin or HMG-CoA reductase inhibitors (atorvastatin, fluvastatin, pitavastatin, pravastatin, rosuvastatin, simvastatin) increases the plasma concentration of each drug, requiring caution for adverse reactions. Co-administration of daclatasvir and sofosbuvir with amiodarone is contraindicated due to a serious risk of severe bradycardia. 
The Korean Association for the Study of the Liver (KASL) 2017 KASL clinical practice guidelines: Management of chronic hepatitis C

\section{Adverse reactions and safety}

The most common adverse reactions observed with daclatasvir in combination with asunaprevir were headache, fatigue, diarrhea, nausea, and alanine aminotransferase (ALT) elevation.

\section{Asunaprevir}

Asunaprevir is an HCV NS3/4A protease inhibitor.

\section{Dosage and administration}

Asunaprevir should be orally administered at the dose of 100 $\mathrm{mg}$ (one capsule) twice a day with or without food.

\section{Pharmacokinetics}

Asunaprevir is metabolized by CYP3A in the liver and mostly eliminated via bile. Compared to subjects with normal hepatic function, the plasma concentration of asunaprevir is not significantly altered in patients with Child-Turcotte-Pugh (CTP) class A cirrhosis, whereas, it can be increased 5-fold and 23-fold in CTP class $B$ and $C$ patients, respectively. Thus, asunaprevir is contraindicated for patients with moderate or severe hepatic impairment (CTP class B or C). No dosage adjustment is required for patients with mild or moderate renal impairment (eGFR 30-80 mL/min). A dosage adjustment of asunaprevir to $100 \mathrm{mg}$ once daily is recommended for patients with severe renal impairment (eGFR $<30 \mathrm{~mL}$ / min) not receiving hemodialysis because the AUC of asunaprevir in those patients increases 2-fold compared with subjects with normal renal function.

\section{Drug-drug interactions}

The drug-drug interactions of asunaprevir with co-medications can be found in the prescribing information of asunaprevir. Coadministration of asunaprevir, an inhibitor of CYP2D6, with antiarrhythmics (flecainide, propafenone) or the antipsychotic thioridazine can cause severe arrhythmia and is thus contraindicated. Strong or moderate CYP3A inducers, including anticonvulsants (carbamazepine, oxcarbazepine, phenobarbital, phenytoin), antituberculosis drugs (rifampin, rifabutin), the endothelin receptor antagonist bosentan, the systemic steroid dexamethasone, the herbal product St. John's wort, antiretrovirals (efavirenz, etravirine, nevirapine), and the stimulant drug modafinil, can decrease the plasma concentration of asunaprevir, with possible loss of efficacy. Thus, co-administration is contraindicated. In addition, strong inhibitors of OATP1B1 or 2B1, such as the antituberculosis drug rifampin, the immunosuppressive drug cyclosporine, and the lipid lowering drug gemfibrozil, can decrease the therapeutic effect of asunaprevir by reducing its intrahepatic levels. Thus, coadministration is not recommended. Strong or moderate CYP3A inhibitors, including antifungals (fluconazole, itraconazole, ketoconazole, posaconazole, voriconazole), macrolides (clarithromycin, erythromycin), calcium channel blockers (diltiazem, verapamil), antiretrovirals (atazanavir, darunavir, fosamprenavir, indinavir, lopinavir, saquinavir), and pharmacokinetic enhancers (cobicistat, ritonavir), can increase plasma levels of asunaprevir and cause severe adverse events. Thus, co-administration is contraindicated. Asunaprevir is a moderate inhibitor of CYP2D6 and an inhibitor of OATP 1B1/1B3 and P-gp. Therefore, co-administration of asunaprevir with substrates of those transporters, including the anticoagulant dabigatran, antiarrhythmics (digoxin, flecainide), HMGCoA reductase inhibitors (atorvastatin, fluvastatin, pitavastatin, pravastatin, rosuvastatin, simvastatin), the antidepressant amitriptyline, and the antitussive dextromethorphan, can increase the plasma levels of each drug. Thus, close clinical monitoring and caution are warranted.

\section{Adverse reactions and safety}

The most common adverse events observed with asunaprevir in combination with daclatasvir were headache, fatigue, diarrhea, nausea, and ALT elevation. The frequency of ALT elevation more than 5 times the upper limit of normal was 3 to $4 \%$, and the frequency of bilirubin elevation more than 2.6 times the upper limit of normal was $1 \%$. Therefore, frequent monitoring of liver function is required to consider possible hepatotoxicity in patients receiving asunaprevir-containing regimens.

\section{Ombitasvir/paritaprevir/ritonavir, dasabuvir}

Ombitasvir is an inhibitor of HCV NS5A; paritaprevir is an HCV NS3/4A protease inhibitor; and ritonavir, a pharmacokinetic enhancer, is an inhibitor of CYP3A4. Dasabuvir is an HCV NS5B polymerase inhibitor.

\section{Dosage and administration}

Ombitasvir/paritaprevir/ritonavir is a fixed-dose combination containing ombitasvir (12.5 mg), paritaprevir (75 mg), and ritonavir $(50 \mathrm{mg}$ ) in a single tablet that should be orally administered at the dose of two tablets once a day with food. Dasabuvir is orally administered at the dose of $250 \mathrm{mg}$ (one tablet) twice a day with food. 


\section{Pharmacokinetics}

Ombitasvir is predominantly metabolized by amide hydrolysis followed by oxidative metabolism. Paritaprevir is predominantly metabolized by CYP3A4, and dasabuvir is metabolized by CYP2C8. Ombitasvir, paritaprevir, and dasabuvir are excreted extensively into the feces. The AUC of paritaprevir increases 1.6-fold in patients with moderate hepatic impairment (CTP class B). The AUC of paritaprevir and dasabuvir increase 9-fold and 3-fold, respectively, in patients with severe hepatic impairment (CTP class C). Therefore, no dosage adjustment is required in patients with mild hepatic impairment (CTP class A). However, this regimen is not recommended for patients with moderate or severe hepatic impairment (CTP class B or C). No dosage adjustment is required in patients with any degree of renal impairment not on dialysis. This regimen has not been studied in patients on dialysis.

\section{Drug-drug interactions}

Moderate or strong CYP3A4 inducers and CYP2C8 inducers decrease the plasma concentration of ombitasvir/paritaprevir/ritonavir and dasabuvir, leading to reduced therapeutic efficacy. Thus, co-administration with anticonvulsants (carbamazepine, oxcarbazepine, phenobarbital, phenytoin), the antituberculosis drug rifampin, the herbal product St. John's wort, the antiretroviral efavirenz, and the endothelin receptor antagonist bosentan is contraindicated. Strong CYP2C8 inhibitors increase the plasma concentration of dasabuvir, which is associated with the risk of QT prolongation; thus, antifungals (itraconazole, ketoconazole, posaconazole, voriconazole), macrolides (clarithromycin, telithromycin), and the lipid lowering drug gemfibrozil are contraindicated. Ritonavir strongly inhibits CYP3A4; paritaprevir inhibits OATP1B1, OATP1B3, BRCP, and P-gp; and dasabuvir and ritonavir inhibit P-gp and BRCP. Co-administration with substrate drugs of those enzymes can increase the plasma concentration of each drug, causing severe adverse events, and is thus not recommended. Those substrate drugs include the angiotensin inhibitor aliskiren, the alpha-adrenoreceptor antagonist alfuzosin, the anti-angina drug ranolazine, antiarrhythmics (amiodarone, dronedarone), the antiplatelet ticagrelor, HMG-CoA reductase inhibitors (atorvastatin, lovastatin, simvastatin), antipsychotics (luracidone, pimozide, quetiapine), the gastrointestinal motility stimulant cisapride, sedatives (triazolam, midazolam), and the ergot agonist ergotamine. Furthermore, serum ALT elevation frequently developed when ombitasvir/paritaprevir/ritonavir and dasabuvir were administered to women using the oral contraceptive ethinylestradiol; thus, co-administration is not recommended. Some other drugs also require caution for dose modification, rescheduling of administration times, or monitoring for adverse reactions caused by drug-drug interactions.

\section{Adverse reactions and safety}

The most common adverse reactions observed with ombitasvir/ paritaprevir/ritonavir and dasabuvir were nausea, pruritus, and insomnia. When it is administered with ribavirin, the most commonly reported adverse reactions were fatigue, nausea, pruritus, other skin reactions, and insomnia. Hepatic decompensation and hepatic failure, including liver transplantation or fatal outcomes, have been reported, mostly in patients with advanced cirrhosis, warranting close monitoring for clinical signs and symptoms. In addition, ALT elevation more than 5 times the upper normal limit at treatment week 4 was reported in about $1 \%$ of patients; thus monitoring of liver enzymes is required.

\section{Elbasvir/grazoprevir}

Elbasvir is an inhibitor of HCV NS5A, and grazoprevir is an HCV NS3/4A protease inhibitor.

\section{Dosage and administration}

Elbasvir/grazoprevir is a fixed dose combination of elbasvir (50 $\mathrm{mg}$ ) and grazoprevir (100 $\mathrm{mg}$ ) in a single tablet and should be orally administered at the dose of one tablet once a day with or without food.

\section{Pharmacokinetics}

Elbasvir and grazoprevir are partially metabolized by CYP3A4 and are mainly eliminated through bile and feces. No dosage adjustment is recommended in patients with mild hepatic impairment (CTP class A). However, the AUC of grazoprevir increases 5-fold and 12-fold in patients with moderate and severe hepatic impairment (CTP class B and C), respectively. Thus, elbasvir/grazoprevir is contraindicated in patients with moderate or severe hepatic impairment (CTP class B or C). No dosage adjustment is recommended in patients with any degree of renal impairment, including patients receiving hemodialysis.

\section{Drug-drug interactions}

Moderate or strong CYP3A inducers, including anticonvulsants (carbamazepine, oxcarbazepine, phenobarbital, phenytoin), the antituberculosis drug rifampin, the herbal product St. John's wort, antiretrovirals (efavirenz, etravirine), the antibiotic nafcillin, the 
The Korean Association for the Study of the Liver (KASL) 2017 KASL clinical practice guidelines: Management of chronic hepatitis C

endothelin receptor antagonist bosentan, and the stimulant drug modafinil, decrease the plasma concentration of elbasvir and grazoprevir; thus, co-administration is contraindicated. Inhibitors of CYP3A or OATP1B1/3, including antiretrovirals (cobicistat-containing regimens, atazanavir, darunavir, lopinavir, saquinavir, tipranavir) and the immunosuppressive drug cyclosporine, can increase the plasma concentration of elbasvir and grazoprevir and are thus contraindicated. Grazoprevir is a weak inhibitor of CYP3A, and elbasvir is a weak inhibitor of P-gp; therefore, the possibility that elbasvir/grazoprevir will affect the plasma levels of other drugs is relatively low. However, co-administration with substrate drugs of CYP3A or P-gp (tacrolimus, statins, dabigatran, ticagrelor) warrants caution for drug-drug interactions.

\section{Adverse reactions and safety}

The most common adverse reactions observed with elbasvir/ grazoprevir were fatigue, headache, and nausea. Serum ALT elevation more than 5 times the upper normal limit at treatment week 8 was reported in about $1 \%$ of patients; thus monitoring for liver function is required.

\section{Glecaprevir/pibrentasvir}

Glecaprevir is an HCV NS3/4A protease inhibitor, and pibrentasvir is an HCV NS5A inhibitor.

\section{Dosage and administration}

Glecaprevir/pibrentasvir is a fixed dose combination of glecaprevir (100 mg) and pibrentasvir (40 mg) in a single tablet and should be orally administered at the dose of three tablets once a day with food.

\section{Pharmacokinetics}

Glecaprevir/pibrentasvir is metabolized by CYP3A and mainly eliminated in bile, with less than $1 \%$ excreted in urine. The AUC of glecaprevir is 2-fold higher in patients with moderate hepatic impairment (CTP class B) compared to normal subjects, and the AUC of glecaprevir and pibrentasvir is 11-fold and 2-fold higher, respectively, in patients with severe hepatic impairment (CTP class C). Therefore, glecaprevir/pibrentasvir is contraindicated in patients with severe hepatic impairment (CTP class C) and is also not recommended in patients with moderate hepatic impairment (CTP class B). No dosage adjustment is recommended in patients with any degree of renal impairment, including patients receiving hemodialysis.

\section{Drug-drug interactions}

Strong or moderate inducers of P-gp and CYP significantly reduce the AUC of glecaprevir/pibrentasvir with loss of therapeutic efficacy. Thus, anticonvulsants (carbamazepine, oxcarbazepine, phenobarbital, phenytoin), antituberculosis drugs (rifampin, rifapentine, rifabutin), the herbal product St. John's wort, the antiretroviral efavirenz, and the endothelin receptor antagonist bosentan are contraindicated. Glecaprevir and pibrentasvir are substrates of P-gp and BCRP, and glecaprevir is a substrate of OATP 1B1/3. Because P-gp, BCRP, and OATP 1B1/3 inhibitors increase the AUC of glecaprevir and pibrentasvir, antiretrovirals (atazanavir, darunavir, lopinavir, ritonavir) are contraindicated, and co-administration with cyclosporine at a dose above $100 \mathrm{mg}$ per day is not recommended.

Glecaprevir and pibrentasvir are inhibitors of P-gp, BCRP, and OATP $1 B 1 / 3$ and can increase the plasma concentrations of drugs that are substrates of those transporters. The angiotensin inhibitor aliskiren, the anticoagulant dabigatran, and HMG-CoA reductase inhibitors (atorvastatin, lovastatin, simvastatin) are contraindicated. Co-administration with other HMG-CoA reductase inhibitors (fluvastatin, pitavastatin, pravastatin, rosuvastatin), the antiarrhythmic digoxin, or immunosuppressive drugs (everolimus, sirolimus, tacrolimus) can increase the plasma concentration of each drug, warranting dose adjustment or monitoring for adverse reactions. Furthermore, serum ALT elevations frequently developed when glecaprevir/pibrentasvir was administered to women using the oral contraceptive ethinylestradiol; thus, glecaprevir/pibrentasvir is not recommended in that condition.

\section{Adverse reactions and safety}

The most common adverse reactions observed with glecaprevir/ pibrentasvir were headache and fatigue.

\section{Sofosbuvir/velpatasvir}

Velpatasvir is an HCV NS5A inhibitor, and sofosbuvir is an HCV NS5B polymerase inhibitor.

\section{Dosage and administration}

Sofosbuvir/velpatasvir is a fixed dose combination of sofosbuvir (400 mg) and velpatasvir (100 mg) in a single tablet and should be orally administered at the dose of one tablet once a day with or without food. 


\section{Pharmacokinetics}

Velpatasvir is metabolized by CYP2B6, CYP2C8, and CYP3A4 and mainly excreted via bile. The AUC of velpatasvir in patients with moderate or severe hepatic impairment is similar to that in subjects with normal hepatic function. Thus, no dosage adjustment is required for patients with cirrhosis, including decompensated disease. No dosage adjustment is required for patients with mild or moderate renal impairment (eGFR $30-80 \mathrm{~mL} / \mathrm{min}$ ), but it is not recommended for use in patients with severe renal impairment (eGFR $<30 \mathrm{~mL} / \mathrm{min}$ ) or ESRD requiring dialysis because the AUC of GS-331007, a metabolite of sofosbuvir, is significantly increased.

\section{Drug-drug interactions}

Strong or moderate inducers of P-gp, CYP2B6, CYP2C8, or CYP3A4 decrease the plasma concentration of sofosbuvir and velpatasvir with loss of therapeutic efficacy. Thus, anticonvulsants (carbamazepine, oxcarbazepine, phenobarbital, phenytoin), antituberculosis drugs (rifampin, rifapentine, rifabutin), the herbal product St. John's wort, the antiretroviral efavirenz, the stimulant modafinil, and the endothelin receptor antagonist bosentan are contraindicated. Because drugs increasing gastric $\mathrm{pH}$ reduce the solubility of sofosbuvir/velpatasvir, caution is needed when antacids, an $\mathrm{H} 2$ receptor antagonist, or a proton pump inhibitor is coadministered.

Velpatasvir is an inhibitor of P-gp, BCRP, OATP1B1/B3, and OATP2B1. Co-administration of sofosbuvir/velpatasvir with substrates of those transporters can increase the plasma concentration of each drug. Sofosbuvir/velpatasvir increases the plasma concentration of topotecan, an anticancer drug; thus, co-administration is not recommended. Co-administration with tenofovircontaining regimens of antiretrovirals or HMG-CoA reductase inhibitors (atorvastatin, rosuvastatin) can increase the plasma levels of each drug, requiring dose adjustment or monitoring for adverse reactions. Co-administration of sofosbuvir/velpatasvir with amiodarone is contraindicated due to a risk of serious bradycardia. Frequent monitoring for digoxin levels is recommended if it is used.

\section{Adverse reactions and safety}

The most common adverse reactions observed with sofosbuvir/ velpatasvir were headache and fatigue. When it is administered with ribavirin, the most commonly reported adverse reactions were fatigue, anemia, nausea, headache, insomnia, and diarrhea.

\section{Sofosbuvir/velpatasvir/voxilaprevir}

Voxilaprevir is an HCV NS3/4A protease inhibitor, velpatasvir is an HCV NS5A inhibitor, and sofosbuvir is an HCV NS5B polymerase inhibitor.

\section{Dosage and administration}

Sofosbuvir/velpatasvir/voxilaprevir is a fixed dose combination of sofosbuvir (400 mg), velpatasvir (100 mg), and voxilaprevir (100 $\mathrm{mg}$ ) in a single tablet and should be orally administered at the dose of one tablet once a day with food.

\section{Pharmacokinetics}

Voxilaprevir is metabolized by CYP3A4 and mainly eliminated via bile. Compared to subjects with normal hepatic function, the AUC of voxilaprevir is 1.7-fold higher in patients with mild hepatic impairment (CTP class A) and 3-fold and 5-fold higher, respectively, in patients with moderate and severe hepatic impairment (CTP class B and C). Therefore, sofosbuvir/velpatasvir/voxilaprevir is contraindicated in decompensated cirrhotic patients with moderate or severe hepatic impairment (CTP class B or C). No dose adjustment is required for patients with mild or moderate renal impairment (eGFR $30-80 \mathrm{~mL} / \mathrm{min}$ ), but it is not recommended for use in patients with severe renal impairment (eGFR $<30 \mathrm{~mL} / \mathrm{min}$ ) or ESRD requiring dialysis because the AUC of GS-331007 is significantly increased.

\section{Drug-drug interactions}

Sofosbuvir, velpatasvir, and voxilaprevir are substrates of P-gp and BCRP, and voxilaprevir is a substrate of OATP1B1/1B3. Strong or moderate P-gp, CYP inducers significantly decrease the AUC of sofosbuvir/velpatasvir/voxilaprevir with loss of therapeutic efficacy. Thus, anticonvulsants (carbamazepine, oxcarbazepine, phenobarbital, phenytoin), antituberculosis drugs (rifampin, rifapentine, rifabutin), the herbal product St. John's wort, the antiretroviral efavirenz, and the endothelin receptor antagonist bosentan are contraindicated. OATP inhibitors can markedly increase the plasma concentration of voxilaprevir; thus, antiretrovirals (atazanavir, lopinavir) and the immunosuppressive drug cyclosporine are contraindicated. Because drugs increasing gastric $\mathrm{pH}$ reduce the solubility of sofosbuvir/velpatasvir/voxilaprevir, caution is warranted when antacids, an $\mathrm{H} 2$ receptor antagonist, or a proton pump inhibitor are co-administered.

Velpatasvir and voxilaprevir are inhibitors of P-gp, BCRP, and OATP 1B1/1B3, and velpatasvir is an inhibitor of OATP 2B1. There- 
The Korean Association for the Study of the Liver (KASL)

2017 KASL clinical practice guidelines: Management of chronic hepatitis C

fore, co-administration with drugs that are substrates of those transporters can change the plasma levels of each drug. HMG$\mathrm{CoA}$ reductase inhibitors (atorvastatin, fluvastatin, lovastatin, pitavastatin, rosuvastatin, simvastatin), anticancer drugs (methotrexate, mitoxantrone, imatinib, irinotecan, lapatinib, topotecan), and the anticoagulant dabigatran are contraindicated. Co-administration of sofosbuvir/velpatasvir/voxilaprevir with amiodarone can cause serious bradycardia and is thus contraindicated. Frequent monitoring for digoxin levels is required when it is used. Co-administration with a tenofovir-containing regimen of antiretrovirals or lipid lowering drugs (ezetimibe, pravastatin) can increase the plasma levels of each drug, requiring dose adjustment or monitoring for adverse reactions. Furthermore, serum ALT elevations frequently developed when sofosbuvir/velpatasvir/voxilaprevir was administered to women using the oral contraceptive ethinylestradiol; thus, sofosbuvir/velpatasvir/voxilaprevir is contraindicated in that condition.

\section{Adverse reactions and safety}

The most common adverse reactions observed with sofosbuvir/ velpatasvir/voxilaprevir were headache, fatigue, diarrhea, and nausea.

\section{HCV RESISTANCE-ASSOCIATED SUBSTITU- TION (RAS) TESTING}

While a variety of DAAs were being tested, amino acid sequence substitutions associated with resistance to DAAs were found (Table 5). ${ }^{9}$ Thus, some DAAs require RAS testing before treatment.

HCV RASs can appear spontaneously during the HCV life cycle without previous DAA therapy. The frequency of naturally occurring RASs varies among genotypes: for an NS3 RAS, it is 75.0\% and $2.0 \%$ in genotypes $1 \mathrm{a}$ and $1 \mathrm{~b}$, respectively; for an NS5A RAS, it is $3.5 \%$ and $14.1 \%$ in genotypes $1 \mathrm{a}$ and $1 \mathrm{~b}$, respectively. ${ }^{10}$ In contrast, the frequency of naturally occurring NS5B RASs is very low: in phase 2 and 3 clinical trials of sofosbuvir $(n=8,598)$, S282T was not detected when measured by the deep sequencing method (with 1\% cutoff). ${ }^{11}$ Viruses resistant to NS3 protease inhibitors or NS5B inhibitors disappear from peripheral blood within a few weeks to months, whereas NS5A inhibitor-resistant viruses persist for years. $^{9}$

There are several RAS testing methods: population sequencing, clonal sequencing, and deep sequencing. The sensitivity of each method is $10-25 \%, 5 \%$, and $0.5-1 \%$, respectively. Resistant viruses present in low proportions (less than 15\%) at baseline do not appear to significantly influence the treatment response. Also, a 15\% cutoff better predicts treatment failure through the selection of resistant viruses. Thus, a 15\% cutoff is widely recommend-

Table 5. Lists of reported RASs in genotype $1 \mathrm{HCV}$

\begin{tabular}{|c|c|c|}
\hline DAAs & HCV subtypes & RASs ${ }^{*}$ \\
\hline \multirow[t]{2}{*}{ NS3/4A protease inhibitors } & $1 \mathrm{a}$ & $\begin{array}{l}\text { V36A/C/G/L/M, Q41R, F43L, T54A/S, V55A/I, Y56H, Q80H/K/L/R, S122R, R155G/I/K/M/S/ } \\
\text { T/W, A156S/T/V, V158I, D168A/C/E/G/H/K/N/T/V/Y, I/V170F/T/V }\end{array}$ \\
\hline & $1 \mathrm{~b}$ & $\begin{array}{l}\text { V36A/C/G/L/M, Q41R, F43I/S/V, T54A/C/G/S, V55A, Y56H/L, Q80H/K/L/R, S122R, R155C/ } \\
\text { G/I/K/Q/M/S/T/W, A156G/F/S/T/V, V158I, D168A/C/E/F/G/H/K/N/T/V/Y, I/V170A/L/T, } \\
\text { M175L }\end{array}$ \\
\hline \multirow[t]{2}{*}{ NS5A inhibitors } & 1a & $\begin{array}{l}\mathrm{K} 24 \mathrm{G} / \mathrm{N} / \mathrm{R}, \mathrm{K} 26 \mathrm{E}, \mathrm{M} 28 \mathrm{~A} / \mathrm{G} / \mathrm{T} / \mathrm{S} / \mathrm{V}, \mathrm{Q} 30 \mathrm{C} / \mathrm{D} / \mathrm{E} / \mathrm{G} / \mathrm{H} / \mathrm{I} / \mathrm{L} / \mathrm{K} / \mathrm{R} / \mathrm{S} / \mathrm{T} / \mathrm{Y}, \mathrm{L} 311 / \mathrm{F} / \mathrm{M} / \mathrm{V}, \mathrm{P} 32 \mathrm{~L} / \mathrm{S}, \mathrm{S} 38 \mathrm{~F}, \\
\mathrm{H} 58 \mathrm{D} / \mathrm{L} / \mathrm{R}, \mathrm{A} 92 \mathrm{~K} / \mathrm{T}, \mathrm{Y93} \mathrm{C} / \mathrm{F} / \mathrm{H} / \mathrm{L} / \mathrm{N} / \mathrm{R} / \mathrm{S} / \mathrm{T} / \mathrm{W}\end{array}$ \\
\hline & $1 b$ & L28M/T, P29S, R30G/H/P/Q, L31I/F/M/V, P32L/S, P58D/S, E62D, A92K, Y93C/H/N/S \\
\hline \multirow{2}{*}{$\begin{array}{l}\text { Nucleotide analogue inhibitors } \\
\text { of NS5B RNA-dependent RNA } \\
\text { polymerase (RdRp) (sofosbuvir) }\end{array}$} & $1 \mathrm{a}$ & L159F, S282T/R, L320F \\
\hline & $1 \mathrm{~b}$ & L159F, S282T \\
\hline \multirow[t]{2}{*}{$\begin{array}{l}\text { Non-nucleoside inhibitors of NS5B } \\
\text { RdRp (dasabuvir) }\end{array}$} & $1 \mathrm{a}$ & $\begin{array}{l}\text { L314H, C316Y, M414T/V, E446K/Q, Y448C/H, C451R, A553T, G554S, Y555H, S556G/R, G557R, } \\
\text { G558R, D559G/N, Y561H/N }\end{array}$ \\
\hline & $1 \mathrm{~b}$ & $\begin{array}{l}\text { C316H/N/Y/W, S368T, N411S, M414I/T/V, C445F/Y, Y448C/H, A553V, G554S, S556G, G558R, } \\
\text { D559G/N }\end{array}$ \\
\hline
\end{tabular}

RAS, resistance-associated substitution; HCV, hepatitis C virus; DAA, direct acting antiviral.

*RASs that confer high-level resistance in the replicon model are in italic. 
ed to report the presence of RASs. ${ }^{9}$ However, RAS testing methods are not yet well standardized, and HCV RAS testing prior to DAA therapy is not generally required in clinical practice.

Exceptionally, several conditions do necessitate RAS testing prior to DAA therapy. For example, in HCV genotype $1 \mathrm{~b}$-infected patients with a baseline NS5A RAS at position L31 or Y93, daclatasvir and asunaprevir combination therapy showed a significantly lower SVR than in those without NS5A RASs. ${ }^{12}$ Therefore, NS5A RAS testing should be performed prior to daclatasvir and asunaprevir combination therapy. If NS5A RASs are detected, an alternative regimen should be considered. In HCV genotype 1a-infected patients with a baseline NS5A RAS at position M28, Q30, L31, or Y93, elbasvir/grazoprevir treatment had a significantly lower SVR than in those without RASs. ${ }^{13}$ Therefore, HCV genotype 1ainfected patients should be tested for NS5A RASs prior to elbasvir/ grazoprevir treatment. If NS5A RASs are detected, the prolongation of treatment duration and the addition of ribavirin should be considered. In addition, RAS testing could help clinicians choose the best drugs for patients who failed with initial DAA therapy. When the HCV titer is very low in serum or a mutation is at the position where the primer binds, a RAS test can be "undetermined." In that case, it is reasonable to regard it as RAS-positive and treat accordingly.

\section{[Recommendations]}

1. Patients with HCV genotype 1b chronic hepatitis $C$ or compensated cirrhosis should be tested for NS5A RASs prior to daclatasvir and asunaprevir combination treatment. If NS5A RASs are detected, an alternative regimen should be considered (A1).

2. Patients with HCV genotype 1a chronic hepatitis C or compensated cirrhosis should be tested for NS5A RASs prior to elbasvir/ grazoprevir treatment. If NS5A RASs are detected, prolonging the treatment duration and adding ribavirin should be considered (A1).

\section{TREATMENT OF CHRONIC HEPATITIS C OR COMPENSATED CIRRHOSIS PATIENTS}

\section{Treatment of chronic hepatitis C or compensated cirrhosis patients with HCV genotype 1 infection}

Several potent DAA oral combination regimens are recommended for patients with genotype 1 infection. However, the regimens differ based on the HCV subtype, the existence of baseline RASs, and liver cirrhosis status. Some regimens have demonstrated higher treatment failure rates in genotype 1a patients. Therefore, genotype 1 patients whose subtype cannot be identified should be treated as if they had a genotype 1a infection.

Few Korean HCV patients have been treated with 1st generation protease inhibitors (PIs) such as boceprevir or telaprevir, except those enrolled in clinical trials. In these guidelines, "treatment-experienced patients" refer to people treated with interferon-based treatments (pegylated or conventional) with or without ribavirin. Those patients might have failed to achieve an SVR for various reasons, including non-response to the treatment or intolerance to or ineligibility for the interferon-based treatment.

\section{Initial treatment of genotype $1 \mathrm{~b}$ patients}

Ledipasvir/sofosbuvir: In a phase 3, open-label study involving previously untreated patients with chronic HCV genotype 1 infection ( $n=865$, genotype $1 \mathrm{~b} 33 \%$, cirrhosis $16 \%$ ), the SVR following 12 weeks of ledipasvir/sofosbuvir treatment was $99 \%$ $(211 / 214) .^{14}$ The addition of ribavirin or treatment extension to 24 weeks did not affect the outcome. In addition, an integrated safety and efficacy analysis of ledipasvir/sofosbuvir in previously untreated patients with genotype 1 HCV infection and compensated cirrhosis ( $n=161$ ) concluded that the addition of ribavirin or extension of treatment duration did not affect the SVR in those patients either. ${ }^{13}$

In a phase 3, open-label study involving 647 previously untreated patients with HCV genotype 1 infection without cirrhosis, the SVR were similar among those who received 8 weeks of ledipasvir/sofosbuvir treatment (94\%, 202/215), 8 weeks of ledipasvir/ sofosbuvir plus ribavirin $(93 \%, 201 / 216)$, and 12 weeks of ledipasvir/sofosbuvir treatment $(95 \%, 206 / 216) .^{15}$ In that study, the degree of fibrosis was assessed by liver biopsy, and cirrhotic patients were excluded. Virologic relapse rates were higher in the 8-week arms with or without ribavirin than in the 12-week treatment group (5\% [20/431] vs. 1\% [3/216]). Post hoc analyses assessed baseline predictors of relapse and identified lower relapse rates in patients receiving 8 weeks of ledipasvir/sofosbuvir who had baseline HCV RNA levels of less than $6,000,000 \mathrm{IU} / \mathrm{mL}$ (2/123; 2\%). In another cohort study, SVRs in patients receiving 8 weeks of ledipasvir/sofosbuvir were not inferior to those in the 12-week treatment group if the patients were non-cirrhotic and had baseline HCV RNA of less than $6,000,000 \mathrm{IU} / \mathrm{mL}$, providing the possibility of shortening therapy for some patients. ${ }^{16}$ In a recent observational study involving HCV genotype $1 \mathrm{~b}$ infected patients without cirrhosis, the SVR rate in the 8-week ledipasvir/so- 
The Korean Association for the Study of the Liver (KASL) 2017 KASL clinical practice guidelines: Management of chronic hepatitis C

fosbuvir treatment group $(97 \%, 62 / 64)$ was similar to that in the 12 week-treatment group $(97 \%, 61 / 63)$ (cirrhotic patients were excluded based on clinical evidence and results from at least two imaging tools, i.e., abdominal ultrasonography, FibroScan ${ }^{\circledR}$, computed tomography, or magnetic resonance imaging). ${ }^{17}$ On the other hand, another observational cohort study found that treatmentnaïve patients with HCV genotype 1 without cirrhosis (FIB- $4 \leq 3.25$ or APRI $\leq 2$ ) and baseline HCV RNA of less than $6,000,000 \mathrm{IU} / \mathrm{mL}$ who completed 8 weeks of ledipasvir/sofosbuvir treatment had an SVR of $93 \%(1,020 / 1,094)$, whereas those who completed 12 weeks of therapy showed an SVR of 97\% (875/906). ${ }^{18}$ However, that study assessed cirrhosis using laboratory markers, FIB-4 or APRI, so the possibility that patients with advanced fibrosis or cirrhosis were recruited cannot be excluded. In another study, in which fibrosis stage was assessed by transient elastography, serum biomarkers (fibro-SURE, Hepascore), or liver biopsy, the SVR of treatment-naïve, HCV genotype 1b infected patients without cirrhosis treated with ledipasvir/sofosbuvir was 99\% (200/202). ${ }^{19}$ In addition, a meta-analysis of six additional cohorts comprising 5,637 patients demonstrated comparable relapse rates after 8-week and 12-week treatments of ledipasvir/sofosbuvir (relative risk [RR] 0.99, 95\% confidence interval [CI] 0.98-1.00). ${ }^{19}$

In treatment-naïve HCV genotype $1 \mathrm{~b}$ infected patients, the presence of baseline NS5A RASs did not affect the treatment outcome after ledipasvir/sofosbuvir treatment, which provided an SVR of $99 \%$ in every group, including those with liver cirrhosis. ${ }^{20}$ However, in a recent cohort study ( $n=772$, treatment naïve $61 \%$ ), NS5A RASs undermined the virological response in HCV genotype $1 \mathrm{~b}$ infected patients with cirrhosis. ${ }^{21}$ In that study, the SVR12 in cirrhotic patients with NS5A RASs $(88 \%$, 49/56) was statistically lower than the SVR12 in the other three groups: non-cirrhosis without NS5A RASs (100\%, 405/405), non-cirrhosis with NS5A RASs (99\%, 125/126), and cirrhosis without NS5A RASs (99\%, 154/155). Although, the researchers did not analyze the SVR according to treatment experience, those findings suggest that baseline RASs could affect treatment outcomes in genotype $1 \mathrm{~b}$ cirrhotic patients.

Elbasvir/grazoprevir: In a phase 3 study, treatment-naïve HCV genotype $1 \mathrm{~b}$ infected patients were treated with elbasvir/ grazoprevir for 12 weeks and showed an SVR of 99\% (129/131). ${ }^{13}$ The presence of compensated cirrhosis did not affect the SVR rates. ${ }^{13}$ The SVR in patients with and without baseline RASs was 94\% (17/18), and 100\% (112/112), respectively. In another phase 3 study, patients infected with HCV genotype 1 or 4 were treated with elbasvir/grazoprevir for 12 weeks (genotype 1b 85\%, PR ex- perience 22\%) and showed an SVR rate of 99\% (128/129). ${ }^{22}$ In that study, HCV genotype $1 \mathrm{~b}$ infected patients with baseline RASs had an SVR of $100 \%(11 / 11) .^{22}$ In a study conducted in Japan, the SVR after patients with genotype $1 \mathrm{~b}$ infection with and without cirrhosis received a 12-week treatment of elbasvir/grazoprevir was 97\% (34/35) and 97\% (219/227), respectively. ${ }^{23}$ In a pooled analysis, HCV genotype $1 \mathrm{~b}$ patients with baseline NS5A RASs who received 12 weeks of elbasvir/grazoprevir had an SVR of $94 \%$ (48/51), and those without baseline RASs had an SVR of $99 \%$ $(247 / 248)(P=0.017){ }^{24}$

Ombitasvir/paritaprevir/ritonavir plus dasabuvir: Treatment-naïve HCV genotype $1 \mathrm{~b}$ infected patients without liver cirrhosis who received ombitasvir/paritaprevir/ritonavir plus dasabuvir combined with ribavirin for 12 weeks showed an SVR of $99 \%$ (209/210), and those who did not receive the ribavirin combination had an SVR of $99 \%$ (207/209). ${ }^{25}$ In another study of patients with genotype $1 \mathrm{~b}$ infection without cirrhosis, the SVR after 12 weeks of therapy with ombitasvir/paritaprevir/ritonavir and dasabuvir with and without ribavirin was 99\% (83/84) and 98\% (81/83), respectively. ${ }^{26}$ In another study, treatment-naïve genotype $1 \mathrm{~b}$ infected patients without liver cirrhosis treated with ombitasvir/paritaprevir/ritonavir plus dasabuvir and ribavirin showed an SVR of $100 \%(22 / 22),{ }^{27}$ as did treatment-naïve genotype $1 \mathrm{~b}$ patients without cirrhosis in a different study who were treated ombitasvir/paritaprevir/ritonavir plus dasabuvir without ribavirin SVR of $100 \%(60 / 60){ }^{28}$

Daclatasvir and sofosbuvir: In a study of 101 patients with HIV/HCV co-infection treated with daclatasvir and sofosbuvir, a post hoc analysis showed that treatment-naïve patients with genotype $1 \mathrm{~b}$ infection without cirrhosis who were treated for 12 weeks showed an SVR of $100 \%(12 / 12) .{ }^{29,30}$ In a study of treatment-naïve patients with genotype 1 infection, the SVR after 24 weeks of treatment with daclatasvir and sofosbuvir was 100\% (29/29), regardless of ribavirin combination, and the SVR after 12 weeks of therapy with daclatasvir and sofosbuvir with and without ribavirin was 95\% (39/41) and 100\% (41/41), respectively. ${ }^{31}$ That study included only 11 patients with cirrhosis, making it inappropriate for evaluating the influence of cirrhosis on SVR. ${ }^{31} \mathrm{~A}$ cohort study that recruited 768 genotype 1 infected patients (liver cirrhosis $73 \%$, genotype $1 \mathrm{~b} 46 \%$, treatment naïve $16 \%$ ) assessed SVR according to the treatment duration (12 weeks vs. 24 weeks of daclatasvir plus sofosbuvir) with and without ribavirin. In cirrhotic patients, the SVR rates after 12-week, 12-week+ribavirin, 24-week, and 24-week+ribavirin treatments were 87\% (82/94), 92\% (23/25), 94\% (323/343), and 98\% (100/102), respectively 
$(P=0.0152)$. That study suggests that cirrhosis status and treatment experience influence SVR. ${ }^{32}$ In a phase 3 study that treated patients with genotype $1 \mathrm{~b}$ infection and decompensated cirrhosis with daclatasvir and sofosbuvir for 12 weeks in combination with ribavirin, the SVR was $100 \%$ (11/11). ${ }^{33}$

Daclatasvir and asunaprevir: Daclatasvir and asunaprevir treatment for 24 weeks in 203 treatment-naïve genotype 1b patients produced an SVR of $90 \%$. $^{12}$ The SVRs did not differ by the baseline factors of gender, age, race, IL28B genotype, or cirrhosis status. However, the presence of baseline NS5A RASs (L31 or Y93) significantly reduced the SVR. A pooled data analysis from 5 phase 2 and 3 studies ( $n=979$, treatment naïve 30\%, liver cirrhosis $22 \%$ ) demonstrated an SVR of $39 \%$ in patients with NS5A RASs compared to an SVR rate of $94 \%$ in patients without RASs after 24 weeks of daclatasvir and asunaprevir treatment. ${ }^{34}$ Baseline NS5A RASs were identified in 13-14\% of the patients included in this analysis. A post hoc analysis of a phase 3 clinical study investigated the efficacy of 24 weeks of asunaprevir and daclatasvir treatment in Asian genotype $1 \mathrm{~b}$ patients ( $n=747$, including 78 Koreans). The SVR was $92 \%$ among treatment-naïve patients. SVR12 varied little according to the baseline factors of age, viral load, IL28 genotype, and cirrhosis status. ${ }^{35}$ A multivariate regression analysis showed that NS5A RAS had a significant influence on SVR (odds ratio [OR] 19.64, 95\% Cl 4.72-81.75).

Glecaprevir/pibrentasvir: In a phase 2 study, DAA-naïve noncirrhotic patients with HCV genotype 1 infection treated with glecaprevir (200 mg) plus pibrentasvir (120 mg) for 12 weeks, glecaprevir (200 mg) plus pibrentasvir (40 mg) for 12 weeks, or glecaprevir (300 mg) plus pibrentasvir (120 mg) for 8 weeks showed SVRs of $100 \%, 97 \%$, and $97 \%$, respectively ( $n=133$, treatment experience $30 \%) .{ }^{36}$ A phase 3 study that assessed the efficacy and safety of 8 and 12 weeks of glecaprevir (300 mg)/pibrentasvir (120 mg) in HCV genotype 1 monoinfected and HIV/HCV co-infected patients without cirrhosis $(n=703$, interferon-based treatment experience $28 \%$, sofosbuvir-based treatment experience $0.4 \%$, genotype 1a $43 \%$ ) showed SVR of $99 \%$ and $99.7 \%$, respectively. ${ }^{37}$ In a pooled analysis of phase 2 and 3 studies of patients with genotype 1-6 chronic HCV infection without cirrhosis (interferon-based treatment experience 23\%, sofosbuvir-based treatment experience 1\%), the SVR rates among patients with genotype 1 infection ( $n=788$, genotype 1 b 56\%) after 8 or 12 weeks of treatment with glecaprevir $(300 \mathrm{mg}$ ) and pibrentasvir (120 mg) were $99 \%$ and $100 \%$, respectively. ${ }^{38}$

A phase 2 study among DAA-naïve HCV genotype 1 infected patients with compensated cirrhosis ( $n=27$, genotype $1 \mathrm{~b} 26 \%$, in- terferon-based treatment experience $22 \%$ ) treated with glecaprevir (200 mg) plus pibrentasvir (120 mg) for 12 weeks showed an SVR of $96 \%{ }^{39}$

A phase 3 study of HCV genotype 1, 2, 4, 5, and 6 infected patients with compensated cirrhosis ( $n=146$, interferon-based treatment experience $17 \%$, sofosbuvir-based treatment experience $8 \%$ ) treated with glecaprevir $(300 \mathrm{mg}) /$ pibrentasvir $(120 \mathrm{mg}$ ) for 12 weeks showed an SVR of $100 \%$ in patients with genotype $1 \mathrm{~b}$ infection $(n=39){ }^{40}$

A phase 3 study conducted in Japan among DAA-naive HCV genotype 1 infected patients without cirrhosis ( $n=129$, genotype 1b 97\%, treatment experience 27\%) treated with glecaprevir (300 $\mathrm{mg}$ )/pibrentasvir (120 mg) for 8 weeks found an SVR of $99 \%{ }^{41}$ On the other hand, treatment-naive HCV genotype 1 infected patients with cirrhosis ( $n=38$, genotype $1 b 100 \%$, treatment experience $32 \%$ ) were treated with glecaprevir $(300 \mathrm{mg}$ )/pibrentasvir $(120 \mathrm{mg})$ for 12 weeks and showed an SVR of $100 \% .^{41}$

Sofosbuvir/velpatasvir: In a phase 3, double-blind, placebocontrolled study among untreated and previously treated HCV genotype 1, 2, 4, 5, and 6 infected patients (including 20\% cirrhotic patients), the SVR of HCV genotype $1 \mathrm{~b}$ infected patients following 12 weeks of treatment with sofosbuvir (400 mg)/velpatasvir (100 mg) was 99\% (117/118). ${ }^{42}$ The SVR was 100\% (94/94) in patients without cirrhosis and 96\% (23/24) in compensated cirrhotic patients. In that study, the SVR of treatment-naïve HCV genotype $1 \mathrm{~b}$ infected patients was 100\% (86/86).

Sofosbuvir/velpatasvir/voxilaprevir: In a phase 3, openlabel trial, HCV infected patients who had not been previously treated with DAAs were randomly assigned to groups given sofosbuvir/velpatasvir/voxilaprevir for 8 weeks or sofosbuvir/velpatasvir for 12 weeks ( $n=941$, conventional or pegylated interferon experienced patients $23 \%$ ). ${ }^{43}$ The overall SVR rate following treatment with sofosbuvir/velpatasvir/voxilaprevir for 8 weeks or sofosbuvir/velpatasvir for 12 weeks was 95\% (476/501) and 98\% (432/440), respectively. Although the 8-week treatment of sofosbuvir/velpatasvir/voxilaprevir seems to be inferior to the 12-week treatment of sofosbuvir/velpatasvir, the HCV genotype $1 \mathrm{~b}$ infected patients treated with sofosbuvir/velpatasvir/voxilaprevir for 8 weeks had an SVR of $97 \%$ (61/63), and those treated with sofosbuvir/velpatasvir for 12 weeks also had an SVR of 97\% (57/59). On the other hand, the HCV genotype 1a infected patients treated for 8 weeks had an SVR of only 92\% (155/169), whereas those treated for 12 weeks had an SVR of $99 \%$ (170/172). The SVRs following an 8-week treatment of sofosbuvir/velpatasvir/voxilaprevir or 12-week treatment of sofosbuvir/velpatasvir were $96 \%$ 
The Korean Association for the Study of the Liver (KASL) 2017 KASL clinical practice guidelines: Management of chronic hepatitis C

(395/411) and 98\% (349/356), respectively, in non-cirrhotic patients and $91 \%(82 / 90)$ and 99\% (83/84) in cirrhotic patients. In HCV genotype $1 \mathrm{~b}$ infected patients, virologic relapse rates were $3 \%(2 / 63)$ after an 8-week treatment of sofosbuvir/velpatasvir/ voxilaprevir and $2 \%(1 / 59)$ after a 12-week treatment of sofosbuvir/velpatasvir. Baseline NS3 or NS5A RASs were found in 50\% (250/501) of patients in the 8-week sofosbuvir/velpatasvir/voxilaprevir treatment group, and those patients had an SVR of $94 \%$ (234/250). On the other hand, baseline NS3 or NS5A RASs were found in 50\% (220/440) of the patients in the sofosbuvir/velpatasvir 12-week treatment group, and their SVR was 99\% (217/220).

\section{Re-treatment of treatment-experienced patients with genotype $1 \mathrm{~b}$ \\ Ledipasvir/sofosbuvir: In a phase 3, randomized, open-label} study among previously treated chronic HCV genotype 1 infected patients ( $n=440$, genotype $1 \mathrm{~b} 21 \%$, cirrhosis $20 \%$, protease inhibitor-experienced patients 53\%), 12 weeks of ledipasvir/sofosbuvir, 12 weeks of ledipasvir/sofosbuvir plus ribavirin, 24 weeks ledipasvir/sofosbuvir, and 24 weeks of ledipasvir/sofosbuvir plus ribavirin provided SVR rates of $87 \%$ (20/23), 100\% (23/23), $100 \%$ (24/24), and $100 \%$ (23/23), respectively. ${ }^{44}$ Baseline viral load, age, IL28B genotype, and sub-genotype had no significant effects on treatment response. In this study, among HCV genotype 1 infected patients with compensated cirrhosis, 12 weeks of ledipasvir/sofosbuvir treatment, 12 weeks of ledipasvir/sofosbuvir plus ribavirin treatment, 24 weeks of ledipasvir/sofosbuvir treatment, and 24 weeks of ledipasvir/sofosbuvir plus ribavirin treatment provided SVR rates of $86 \%$ (19/22), 82\% (18/22), 95\% $(21 / 22)$, and $100 \%(22 / 22)$, respectively $(P=0.007)$. In another study where treatment-experienced HCV genotype 1 infected patients with compensated cirrhosis that were enrolled in phase 2 or 3 clinical trials were analyzed ( $n=352$, genotype 1 b 37\%), 12 weeks of ledipasvir/sofosbuvir treatment, 12 weeks ledipasvir/sofosbuvir plus ribavirin treatment, 24 weeks of ledipasvir/sofosbuvir treatment, and 24 weeks of ledipasvir/sofosbuvir plus ribavirin treatment provided SVR rates of 90\% (64/71), 96\% (152/159), $98 \%(98 / 100)$, and $100 \%(22 / 22)$, respectively, showing that the addition of ribavirin and extended treatment duration improved the virologic response. ${ }^{13}$ On the other hand, in a study involving PI-experienced cirrhotic patients ( $n=155$, genotype $1 \mathrm{~b} 55 \%$ ), the SVR of the 12-week ledipasvir/sofosbuvir plus ribavirin treatment group $(96 \%, 74 / 77)$ was similar to that of the 24-week ledipasvir/ sofosbuvir plus ribavirin treatment group $(97 \%, 75 / 77) .{ }^{45}$ Howev- er, a phase 3 Japanese study ( $n=341$, genotype $1 \mathrm{~b} 97 \%$, cirrhosis $22 \%$, treatment experience $51 \%$ ) found that patients treated for 12 weeks with ledipasvir/sofosbuvir without ribavirin and those treated for 12 weeks with ledipasvir/sofosbuvir plus ribavirin showed SVR rates of $100 \%$ (171/171) and 98\% (167/170), respectively. ${ }^{46}$ Therefore, HCV genotype $1 \mathrm{~b}$ infected patients treated with ledipasvir/sofosbuvir without ribavirin for 12 weeks showed a high SVR irrespective of their treatment experience or cirrhosis status. In a phase 3 Korean study, treatment-experienced, HCV genotype 1 infected patients ( $n=47$, genotype 1b 98\%, cirrhosis $28 \%$ ) treated with ledipasvir/sofosbuvir for 12 weeks showed an SVR of $98 \%(46 / 47))^{47}$

It has been recognized that the presence of NS5A RASs might not affect the SVRs in genotype $1 \mathrm{~b}$ patients. ${ }^{46,48}$ However, a recent study reported that the presence of NS5A RASs did affect the SVR in genotype $1 \mathrm{~b}$ patients ${ }^{21}$ and a study reported that among treatment-experienced patients. the SVR was 89\% (41/46) in NS5A RAS-positive patients and 98\% (267/272) in NS5A RASnegative patients. ${ }^{20}$

Elbasvir/grazoprevir: In a phase 3 study, treatment-experienced genotype 1 infected patients (genotype 1b 35\%, liver cirrhosis 35\%) treated with elbasvir/grazoprevir without ribavirin for 12 weeks had an SVR of $100 \%$ (34/34), and those treated with elbasvir/grazoprevir plus ribavirin for 12 weeks had an SVR of 97\% (28/29), whereas those treated with elbasvir/grazoprevir without ribavirin for 16 weeks had an SVR of 100\% (36/36), and those treated for 16 weeks with elbasvir/grazoprevir plus ribavirin had an SVR of $98 \%(46 / 47) .{ }^{49}$ The presence of baseline NS5A RASs did not affect the SVRs in patients with genotype $1 \mathrm{~b}$ infection treated with 12 weeks of elbasvir/grazoprevir. ${ }^{49}$ In a pooled analysis, HCV genotype $1 \mathrm{~b}$ patients with baseline NS5A RASs treated with 12 weeks of elbasvir/grazoprevir had an SVR of $94 \%$ (48/51), and those without baseline RASs had an SVR of $99 \%$ $(247 / 248)(P=0.017){ }^{24}$

Ombitasvir/paritaprevir/ritonavir plus dasabuvir: In treatment-experienced patients with genotype $1 \mathrm{~b}$ infection without cirrhosis, the SVR after a 12-week treatment with ombitasvir/ paritaprevir/ritonavir plus dasabuvir with ribavirin was $97 \%$ $(119 / 123) .{ }^{50}$ In another study of treatment-experienced patients with genotype $1 \mathrm{~b}$ infection, the SVR after a 12-week treatment of ombitasvir/paritaprevir/ritonavir plus dasabuvir with and without ribavirin was 97\% (85/88) and 97\% (85/88), respectively. The addition of ribavirin was thus not beneficial in treatment-experienced genotype $1 \mathrm{~b}$ infected patients. ${ }^{51}$ In a phase 3 study of 380 patients with genotype 1 infection and cirrhosis (treatment expe- 
rience $58 \%$, genotype $1 \mathrm{~b} 31 \%$ ), the SVR of genotype $1 \mathrm{~b}$ infected patients treated with ombitasvir/paritaprevir/ritonavir plus dasabuvir with ribavirin for 12 or 24 weeks was $99 \%$ (67/68) and $100 \%$ (51/51), respectively. ${ }^{27}$ In another study of 60 genotype $1 \mathrm{~b}$ infected patients with cirrhosis (treatment experience $55 \%$ ), the SVR of ombitasvir/paritaprevir/ritonavir plus dasabuvir treatment for 12 weeks was $100 \%(60 / 60){ }^{28}$

Daclatasvir and sofosbuvir: In a study of treatment-experienced patients with HIV/HCV co-infection, genotype $1 \mathrm{~b}$ infected patients treated with daclatasvir and sofosbuvir for 12 weeks showed an SVR of 98\% (43/44). ${ }^{30}$ That study included 15 patients with cirrhosis whose subtypes were not analyzed. ${ }^{30}$ In a phase 2 study of 41 treatment-experienced patients with genotype 1 infection (genotype $1 \mathrm{~b} 24 \%$, cirrhosis $14 \%$ ), the SVR from a 24 week treatment of daclatasvir and sofosbuvir with and without ribavirin were $100 \%(21 / 21)$ and $95 \%$ (19/20), respectively. ${ }^{31} \mathrm{~A}$ cohort study of 768 genotype 1 infected patients (liver cirrhosis $73 \%$, genotype $1 \mathrm{~b} 46 \%$, treatment experience $84 \%$ ) assessed SVR by treatment duration (12 weeks vs. 24 weeks of daclatasvir and sofosbuvir) with and without ribavirin. In cirrhotic patients, the SVR following 12 weeks, 12 weeks+ribavirin, 24 weeks, and 24 weeks+ribavirin was $87 \%$ (82/94), 92\% (23/25), 94\% (323/343), and 98\% (100/102), respectively. ${ }^{32}$ That study revealed that cirrhosis and treatment experience influenced SVR. ${ }^{32} \mathrm{~A}$ phase 3 study treated patients with genotype $1 \mathrm{~b}$ infection and decompensated cirrhosis with daclatasvir and sofosbuvir for 12 weeks in combination with ribavirin and found an SVR of 100\% (11/11). ${ }^{33}$

Daclatasvir and asunaprevir: A phase 3 study that evaluated the efficacy of 24 weeks of treatment with daclatasvir and asunaprevir in previous non-responders to peginterferon alpha plus ribavirin and those ineligible for or intolerant to peginterferon alpha plus ribavirin demonstrated an SVR of 82\% (168/205) and $82 \%$ (192/235), respectively, whereas the SVR in treatment-naïve patients following the same protocol was $90 \%(182 / 203))^{12}$ A post hoc analysis of that study to determine the efficacy of that treatment in Korean patients $(n=44)$ showed an SVR of 70\% (16/23) in non-responders and 86\% (15/21) in patients ineligible for or intolerant to peginterferon-based treatment. ${ }^{35}$ The presence of NS5A RASs appeared to be the single most important predictor of treatment failure in that study. Another phase 3 study conducted in Japan among 87 non-responders and 135 patients ineligible for or intolerant to peginterferon-based treatment received daclatasvir and asunaprevir treatment for 24 weeks and provided an overall SVR of $85 \%(188 / 222) .^{52}$ In that study, non-responders showed an SVR of $81 \%$ (70/87), and patients ineligible for or intolerant to peginterferon-based treatment had an SVR of 87\% (118/135). Baseline cirrhosis status had no effect on SVR, and baseline NS5A RASs were not tested in that study. A meta-analysis of 9 trials ( $n=1690$ ) that provide 24 week treatment of asunaprevir and daclatasvir showed an SVR of 90\% (336/374) in treatment-naïve patients and $82 \%$ (316/386) and 85\% (514/607) in non-responders and patients ineligible for or intolerant to peginterferon-based treatments, respectively. ${ }^{53}$ However, that study did not analyze the presence of baseline NS5A RASs, so enrolled patients might have had baseline NS5A RASs. Recently, Korean genotype $1 \mathrm{~b}$ patients without baseline NS5A RASs (treatment experience 25\%, cirrhosis 29\%) were treated with daclatasvir and asunaprevir for 24 weeks. The patients without liver cirrhosis had an SVR of $98 \%$ (102/104), and those with compensated liver cirrhosis had an SVR of $92 \%(44 / 48)(P=0.080) .{ }^{54}$ Asunaprevir and daclatasvir were given for 24 weeks in Korean genotype $1 \mathrm{~b}$ patients without baseline NS5A RASs ( $n=251$, treatment experience $36 \%$, cirrhosis $22 \%)$ and provided an overall SVR of $98 \%$ (246/251), whereas patients with liver cirrhosis showed an SVR of $96 \%(49 / 51){ }^{55}$

Glecaprevir/pibrentasvir: In a phase 2 study recruiting DAA-naïve genotype 1-6 infected patients without cirrhosis, genotype 1 infected patients ( $n=133$, treatment experience $30 \%$ ) treated with glecaprevir $(200 \mathrm{mg}$ ) plus pibrentasvir $(120 \mathrm{mg})$ for 12 weeks, glecaprevir (200 mg) plus pibrentasvir $(40 \mathrm{mg}$ ) for 12 weeks, or glecaprevir (300 mg) plus pibrentasvir (120 mg) for 8 weeks showed SVR rates of $100 \%, 97 \%$, and $97 \%$, respectively. ${ }^{36}$ In a phase 3 study that assessed the efficacy and safety of 8 or 12 weeks of glecaprevir (300 mg)/pibrentasvir (120 mg) in genotype 1 mono-infected or HIV/HCV co-infected patients without cirrhosis ( $n=703$, interferon-based treatment experience $28 \%$, sofosbuvir-based treatment experience $0.4 \%$, genotype 1a $43 \%$ ), the SVRs were $99 \%$ and $99.7 \%$, respectively. ${ }^{37}$ In a pooled analysis of phase 2 and 3 studies of genotype 1-6 HCV infected patients without cirrhosis (interferon-based treatment experience $23 \%$, sofosbuvir-based treatment experience 1\%), HCV genotype 1 infected patients ( $n=788$, genotype $1 \mathrm{~b} 56 \%$ ) treated with glecaprevir $(300 \mathrm{mg})$ and pibrentasvir $(120 \mathrm{mg})$ for 8 or 12 weeks had SVRs of $99 \%$ and $100 \%$, respectively. ${ }^{38}$

In a phase 2 study recruiting DAA-naïve genotype 1 infected patients with compensated cirrhosis ( $n=27$, genotype $1 \mathrm{~b} 26 \%$, interferon-based treatment experience 22\%), the SVR rate after 12 weeks of glecaprevir $(200 \mathrm{mg}$ ) plus pibrentasvir $(120 \mathrm{mg}$ ) was $96 \%{ }^{39}$ In a phase 3 study that assessed the efficacy and safety of 12 weeks of glecaprevir $(300 \mathrm{mg}) /$ pibrentasvir $(120 \mathrm{mg})$ in genotype 1, 2, 4, 5, and 6 HCV patients with compensated cirrhosis 
The Korean Association for the Study of the Liver (KASL) 2017 KASL clinical practice guidelines: Management of chronic hepatitis C

( $n=146$, interferon-based treatment experience 17\%, sofosbuvirbased treatment experience $8 \%$ ), genotype $1 \mathrm{~b}$ infected patients $(n=39)$ had an SVR of $100 \%{ }^{40}$

In a phase 3 study conducted in Japan among DAA-naive genotype 1 infected patients, patients without cirrhosis $(n=129$, genotype $1 \mathrm{~b} 97 \%$, treatment experience $27 \%$ ) were treated with glecaprevir (300 mg)/pibrentasvir (120 mg) for 8 weeks and showed an SVR of $99 \%$, whereas those with cirrhosis ( $n=38$, genotype $1 b$ $100 \%$, treatment experience $32 \%$ ) were treated with glecaprevir (300 mg)/pibrentasvir (120 mg) for 12 weeks and showed an SVR of $100 \%{ }^{41}$

Sofosbuvir/velpatasvir: Treatment-experienced HCV genotype 1 infected patients were treated with sofosbuvir plus velpatasvir (25 mg or $100 \mathrm{mg}$ ) with or without ribavirin for 12 weeks.In this study, HCV genotype 1 patients treated with sofosvuvir plus velpatasvir (100 mg) with or without ribavirn showed SVRs of $100 \%$ (27/27) and 96\% (27/28), respectively. ${ }^{56}$

In a phase 3, double-blind, placebo-controlled study involving untreated and previously treated HCV genotype 1, 2, 4, 5, or 6 infected patients with or without compensated cirrhosis, previously treated HCV genotype 1b patients treated with sofosbuvir (400 $\mathrm{mg}$ )/velpatasvir (100 mg) for 12 weeks showed an SVR of $97 \%$ $(31 / 32)^{42}$

Sofosbuvir/velpatasvir/voxilaprevir: In a phase 3, openlabel trial, HCV infected patients who had not previously been treated with a DAA were randomly assigned to groups given 8 weeks of sofosbuvir/velpatasvir/voxilaprevir treatment or 12 weeks of sofosbuvir/velpatasvir treatment $(n=941$, conventional or pegylated interferon experience $23 \%$ ). ${ }^{43}$ The overall SVR in the 8-week sofosbuvir/velpatasvir/voxilaprevir group and 12-week sofosbuvir/velpatasvir group was 95\% (476/501) and 98\% (432/440), respectively. Although the 8-week treatment of sofosbuvir/velpatasvir/voxilaprevir seems to be inferior to the 12-week treatment of sofosbuvir/velpatasvir, the HCV genotype $1 \mathrm{~b}$ infected patients treated with sofosbuvir/velpatasvir/voxilaprevir for 8 weeks had an SVR of $97 \%(61 / 63)$, and those treated with sofosbuvir/velpatasvir for 12 weeks also showed an SVR of $97 \%$ (57/59). On the other hand, HCV genotype 1a infected patients treated for 8 weeks had an SVR of only 92\% (155/169), and those treated for 12 weeks had an SVR of $99 \%$ (170/172). The SVR rates following the 8-week treatment of sofosbuvir/velpatasvir/voxilaprevir and 12-week treatment of sofosbuvir/velpatasvir were $96 \%$ (395/411) and 98\% (349/356), respectively, in non-cirrhotic patients, and 91\% (82/90) and 99\% (83/84) in cirrhotic patients. In HCV genotype $1 \mathrm{~b}$ infected patients, virologic relapse rates were
$3 \%(2 / 63)$ after the 8-week treatment of sofosbuvir/velpatasvir/ voxilaprevir and $2 \%(1 / 59)$ after the 12 -week treatment of sofosbuvir/velpatasvir.

\section{[Recommendations] (Table 6)}

Initial treatment of chronic hepatitis $\mathrm{C}$ or compensated cirrhosis patients with $\mathrm{HCV}$ genotype $1 \mathrm{~b}$ infection

1. Ledipasvir/sofosbuvir should be administered for 12 weeks (A1). Treatment could be shortened to 8 weeks in patients without liver cirrhosis and without HIV co-infection whose baseline HCV RNA level is less than $6,000,000 \mathrm{IU} / \mathrm{mL}$ (B1).

2. Elbasvir/grazoprevir should be administered for 12 weeks (A1).

3. Ombitasvir/paritaprevir/ritonavir plus dasabuvir should be administered for 12 weeks (A1).

4. Daclatasvir and sofosbuvir should be administered for 12 weeks to patients without liver cirrhosis (A1). Daclatasvir, sofosbuvir, and ribavirin could be administered for 12 weeks to patients with liver cirrhosis, or they could receive daclatasvir and sofosbuvir for 24 weeks (B1).

5. Daclatasvir and asunaprevir could be administered for 24 weeks to patients without baseline NS5A RASs (A2). Patients with baseline NS5A RASs should be treated with other regimens (A1).

6. Glecaprevir/pibrentasvir should be administered for 8 weeks to patients without liver cirrhosis (A1). Glecaprevir/pibrentasvir should be administered for 12 weeks to patients with liver cirrhosis (A1).

7. Sofosbuvir/velpatasvir should be administered for 12 weeks (A1).

Retreatment of treatment-experienced chronic hepatitis $C$ or compensated cirrhosis patients with HCV genotype $1 \mathrm{~b}$ infection 1. Ledipasvir/sofosbuvir should be administered for 12 weeks to patients without liver cirrhosis (A1)

Ledipasvir/sofosbuvir and ribavirin could be administered for 12 weeks to patients with liver cirrhosis, or they could receive ledipasvir/sofosbuvir for 24 weeks (B1).

2. Elbasvir/grazoprevir should be administered for 12 weeks (A1).

3. Ombitasvir/paritaprevir/ritonavir plus dasabuvir should be administered for 12 weeks (A1)

4. Daclatasvir and sofosbuvir sould be administered for 12 weeks to patients without liver cirrhosis (A1)

Daclatasvir, sofosbuvir, and ribavirin could be administered for 12 weeks to patients with liver cirrhosis, or they could receive daclatasvir and sofosbuvir for 24 weeks (B1).

5. Daclatasvir and asunaprevir could be administered to patients without baseline NS5A RASs for 24 weeks (A2).

Patients with baseline NS5A RASs should be treated with other regimens (A1).

6. Glecaprevir/pibrentasvir should be administered for 8 weeks to patients without liver cirrhosis (A1)

Glecaprevir/pibrentasvir should be administered for 12 weeks to patients with liver cirrhosis (A1).

7. Sofosbuvir/velpatasvir should be administered for 12 weeks (A1). 


\section{Initial treatment of HCV genotype 1a patients}

\section{Ledipasvir/sofosbuvir}

In a phase 3, open-label study recruiting previously untreated HCV genotype 1 infected patients ( $n=865$, genotype 1a $67 \%$, cirrhosis 16\%), the SVR after a 12-week treatment with ledipasvir/ sofosbuvir, 12-week treatment with ledipasvir/sofosbuvir plus ribavirin, 24-week treatment of ledipasvir/sofosbuvir, and 24-week treatment of ledipasvir/sofosbuvir plus ribavirin were $99 \%$ (211/214), 97\% (211/217), 98\% (212/217), and 99\% (215/217), respectively. ${ }^{14}$ No significant differences in SVR were found according to age, baseline HCV RNA level, cirrhosis status, IL28B genotype, or HCV sub-genotype (1a vs. 1b).

A post hoc analysis of data from seven clinical trials to evaluate the efficacy and safety of ledipasvir/sofosbuvir with and without ribavirin included 161 treatment-naïve patients with genotype 1 and compensated cirrhosis (genotype 1a 53\%). ${ }^{57}$ The SVR following a 12-week treatment of ledipasvir/sofosbuvir, 12-week treatment of ledipasvir/sofosbuvir plus ribavirin, 24-week treatment of ledipasvir/sofosbuvir, and 24-week treatment of ledipasvir/sofosbuvir plus ribavirin were 96\% (45/47), 98\% (44/45), 97\% (32/33), and $100 \%(36 / 36)$, respectively. SVR did not vary significantly with treatment duration (12 weeks $97 \%$ [89/92] vs. 24 weeks $99 \%$ [68/69]), presence/absence of ribavirin (99\% [80/81] vs. $96 \%$ [77/80]), HCV sub-genotype (1a $98 \%$ [84/86] vs. 1b 97\% [72/74]), or IL28B genotype (CC 98\% (56/57) vs. non-CC 97\% [101/104]).

Twelve- or 24-week treatment with ledipasvir/sofosbuvir with or without ribavirin provided an overall SVR of 91\% (42/46) in patients with baseline ledipasvir-specific RASs (NS5A positions 24, $28,30,31,32,38,58,92,93)$ and an SVR of $99 \%(539 / 546)$ in those without ledipasvir-specific RASs. ${ }^{20}$ In cirrhotic patients, the
SVR was $86 \%(6 / 7)$ in patients with baseline ledipasvir-specific RASs and 99\% (71/73) in those without ledipasvir-specific RASs.

In a phase 3, open-label study of 647 previously untreated HCV genotype 1 infected patients without cirrhosis (genotype 1a $80 \%$ ), the SVR following an 8-week treatment of ledipasvir/sofosbuvir, 8-week treatment of ledipasvir/sofosbuvir plus ribavirin, or 12-week treatment of ledipasvir/sofosbuvir were 94\% (202/215), 93\% (201/216), and 95\% (206/216), respectively. ${ }^{15}$ Virologic relapse rates were higher in the 8-week treatment arms than in the 12-week treatment arm (5\% [20/431] vs. 1\% [3/216]). Post hoc analyses assessed baseline predictors of relapse and identified a low relapse rate in patients receiving 8 weeks of ledipasvir/sofosbuvir who had baseline HCV RNA levels $<6,000,000 \mathrm{IU} / \mathrm{mL}$ $(2 / 123 ; 2 \%)$. The same held true for patients with similar baseline HCV RNA levels who received 12 weeks of treatment $(2 / 131 ; 2 \%)$.

Treatment-naïve HCV genotype 1 infected patients without cirrhosis (defined as FIB-4 $\leq 3.25$ ) and with baseline HCV RNA< $6,000,000 \mathrm{IU} / \mathrm{mL}$ (genotype 1a > 70\%) showed a lower SVR [93.2\% $(1,020 / 1,094)]$ when treated with ledipasvir/sofosbuvir for 8 weeks compared to those treated with ledipasvir/sofosbuvir for 12 weeks $(97 \%[875 / 906]) .^{18}$

However, another study found that the SVR of treatment-naïve HCV genotype 1a infected patients without cirrhosis whose baseline HCV RNA<6,000,000 IU/mL was 98\% (372/381). ${ }^{19}$

\section{Elbasvir/grazoprevir}

In a phase 3 study that evaluated the efficacy of a 12-week treatment with elbasvir/grazoprevir in treatment-naïve genotype 1a infected patients, the SVR was $92 \% .^{13}$ The SVR in patients with compensated cirrhosis was $97 \%$, which did not differ from that in patients without cirrhosis. ${ }^{13,58}$ SVR did not differ by age, gender,

Table 6. Treatment of chronic hepatitis C or compensated cirrhosis patients with HCV genotype 1b infection

\begin{tabular}{|c|c|c|c|c|}
\hline & \multicolumn{2}{|c|}{ Treatment naive } & \multicolumn{2}{|c|}{ PR experienced } \\
\hline & Chronic hepatitis & Compensated cirrhosis & Chronic hepatitis & Compensated cirrhosis \\
\hline Ledipasvir/sofosbuvir & $12 w k\left(8 w k^{*}\right)$ & $12 w k$ & $12 w k$ & $12 w k+R / 24 w k$ \\
\hline Elbasvir/grazoprevir & $12 w k$ & $12 w k$ & $12 w k$ & $12 w k$ \\
\hline Ombitasvir/paritaprevir/ritonavir+dasabuvir & $12 w k$ & $12 w k$ & $12 w k$ & $12 w k$ \\
\hline Daclatasvir+sofosbuvir & $12 w k$ & $12 w k+R / 24 w k$ & $12 w k$ & $12 w k+R / 24 w k$ \\
\hline Daclatasvir+asunaprevir & $24 w k$ & $24 w k$ & $24 w k$ & $24 w k$ \\
\hline Glecaprevir/pibrentasvir & $8 w k$ & $12 w k$ & $8 w k$ & $12 w k$ \\
\hline Sofosbuvir/velpatasvir & $12 w k$ & $12 w k$ & $12 w k$ & $12 w k$ \\
\hline
\end{tabular}

HCV, hepatitis C virus; $P R$, pegylated interferon alpha+ribavirin; $R$, weight-based ribavirin; wk, weeks.

*Without liver cirrhosis, without human immunodeficiency virus co-infection, and HCV RNA level of less than 6,000,000 IU/mL. 
The Korean Association for the Study of the Liver (KASL) 2017 KASL clinical practice guidelines: Management of chronic hepatitis C

race, or IL28B genetic polymorphism, but it was higher in patients with HCV RNA $\leq 800,000 \mathrm{lU} / \mathrm{mL}$ than in those with HCV RNA > $800,000 \mathrm{IU} / \mathrm{mL}$ (100\% vs. 92\%). NS5A RASs were detected in $12 \%$ of the genotype 1a infected patients, and the SVR was significantly lower in patients with RASs than in those without them (58\% vs. 99\%). However, the SVR was 100\% (17/17) even in patients with RASs when pretreatment HCV RNA was $\leq 800,000 \mathrm{IU} / \mathrm{mL}{ }^{13} \mathrm{~A}$ pooled analysis of phase 2 and 3 clinical trials revealed that the SVR was $100 \%$ in genotype 1a infected patients with baseline NS5A RASs treated with elbasvir/grazoprevir and ribavirin for 16 or 18 weeks. ${ }^{59,60}$ Another pooled analysis reported that NS5A RASs (M29, Q30, L31, or Y93 mutation) were detected in 11\% (92/825) of treatment-naïve genotype 1a infected patients. The SVR was significantly lower in patients with RASs (73\% [33/45]) than in those without them (98\% [364/371]) following treatment with elbasvir/grazoprevir for 12 weeks. Treatment with elbasvir/ grazoprevir plus ribavirin for 16 or 18 weeks provided an SVR of $100 \%$ in treatment-naïve HCV genotype 1a infected patients with baseline RASs. $^{24}$

\section{Ombitasvir/paritaprevir/ritonavir plus dasabuvir}

In treatment-naïve patients with genotype 1a infection but not cirrhosis, the SVR after treatment for 12 weeks with a combination of ombitasvir/paritaprevir/ritonavir plus dasabuvir with ribavirin was 95\% (306/322). ${ }^{61}$ In another study of treatment-naïve HCV genotype 1 infected patients without cirrhosis, the SVR after ombitasvir/paritaprevir/ritonavir and dasabuvir treatment without ribavirin for 12 weeks was $99 \%$ in genotype $1 \mathrm{~b}$ patients, whereas it was $90 \%$ in genotype 1a patients. ${ }^{25}$ The SVR after receiving a combination of ombitasvir/paritaprevir/ritonavir, dasabuvir, and ribavirin for 12 or 24 weeks was 89\% (124/140) and 94\% (114/121), respectively, in treatment-naïve and treatment-experienced HCV genotype 1a patients with compensated cirrhosis. ${ }^{27}$

\section{Daclatasvir and sofosbuvir}

In a phase 3 study that assessed the efficacy of a 12-week treatment of daclatasvir and sofosbuvir in treatment-naïve HCV genotype 1 infected patients (genotype 1a 71, 1b 12, compensated cirrhosis 9), the SVR was $96 \%{ }^{30}$ In a phase 2 study of treatment-naïve genotype 1 infected patients ( $n=126$, genotype 1a 99, 1b 27), the SVR after 12 or 24 weeks of daclatasvir and sofosbuvir treatment was 100\% (41/41) and 100\% (29/29) respectively, and the SVR after 12 or 24 weeks of treatment with daclatasvir and sofosbuvir with ribavirin was 95\% (39/41) and 100\% (15/15), with no statistical differences by treatment duration or the addition of ribavirin. ${ }^{31}$ However, only 14 genotype 1 infected patients with compensated cirrhosis were included in that study. In a cohort study of 768 genotype 1 infected patients (liver cirrhosis 73\%, CTP classification B or C decompensated cirrhosis 3\%, genotype 1a $50 \%$, treatment naïve $16 \%$ ), the SVR after 12 or 24 weeks of treatment with daclatasvir and sofosbuvir was 92\% (147/160) and $95 \%$ (417/439), respectively, and the SVR after 12 or 24 weeks of treatment with daclatasvir and sofosbuvir with ribavirin was $94 \%$ (32/34) and 99\% (133/135), respectively, with no statistical differences among treatment groups. However, the SVR was lower in patients with cirrhosis than in those without cirrhosis $(88 \%$ [105/119] vs. 95\% [423/444], $P=0.0054) .{ }^{32}$ Although studies on the combination of daclatasvir and sofosbuvir are insufficient in treatment-naïve genotype 1a infected patients with compensated cirrhosis, given the lower SVR in cirrhotic patients and risk of emerging NS5A RASs in cases of treatment failure, 12-week treatment with daclatasvir and sofosbuvir with ribavirin or 24-week treatment with daclatasvir and sofosbuvir can be considered.

\section{Glecaprevir/pibrentasvir}

In a phase 2 study for DAA-naïve, HCV genotype 1-6 infected patients without cirrhosis, the SVR after treatment with glecaprevir (200 mg) plus pibrentasvir (120 mg) for 12 weeks, glecaprevir (200 mg) plus pibrentasvir $(40 \mathrm{mg}$ ) for 12 weeks, or glecaprevir (300 mg) plus pibrentasvir (120 mg) for 8 weeks was 100\%, $97 \%$, and $97 \%$, respectively, in genotype 1 infected patients $(n=133$, treatment experience $30 \%) .^{36}$ In a phase 3 study that assessed the efficacy and safety of 8- and 12-week treatment with glecaprevir (300 mg)/pibrentasvir (120 mg) in genotype 1 monoinfected or HIV/HCV co-infected patients without cirrhosis $(n=703$, interferon-based treatment experience $28 \%$, sofosbuvir-based treatment experience $0.4 \%$ ), the SVR was $99 \%$ and $99.7 \%$, respectively. ${ }^{37}$ In a pooled analysis of phase 2 and 3 studies of genotype 1-6 chronic HCV infected patients without cirrhosis (interferon-based treatment experience 23\%, sofosbuvir-based treatment experience 1\%), the SVR after treatment with glecaprevir (300 mg) plus pibrentasvir (120 mg) for 8 or 12 weeks was 99\% and 100\%, respectively, in HCV genotype 1 infected patients without cirrhosis ( $n=788$, genotype 1a 44\%).

In a phase 2 study of DAA-naïve HCV genotype 1 infected patients with compensated cirrhosis $(n=27$, interferon-based treatment experience $22 \%$ ), the SVR after a 12-week treatment with glecaprevir (200 mg) plus pibrentasvir (120 mg) was 96\%. ${ }^{39}$

In a phase 3 study that assessed the efficacy and safety of a 12week treatment with glecaprevir $(300 \mathrm{mg}$ )/pibrentasvir $(120 \mathrm{mg}$ ) 
in patients infected with HCV genotype 1, 2, 4, 5, or 6 and compensated cirrhosis $(n=146$, interferon-based treatment experience $17 \%$, sofosbuvir-based treatment experience $8 \%$ ), the SVR was $99 \%$ in HCV genotype 1a infected patients $(n=48) .{ }^{40}$

\section{Sofosbuvir/velpatasvir}

In a phase 3, double-blind, placebo-controlled study recruiting untreated and previously treated patients with HCV genotype 1, 2, 4,5 , or 6 , including those with compensated cirrhosis, the SVR after a 12-week treatment with sofosbuvir (400 mg)/velpatasvir (100 mg) was 98\% (157/161) in HCV genotype 1a infected patients without cirrhosis and 100\% (49/49) in those with compensated cirrhosis. The SVR of treatment-naïve HCV genotype 1a infected patients was $97 \%(128 / 132){ }^{42}$

\section{Sofosbuvir/velpatasvir/voxilaprevir}

In a phase 3, open-label trial, HCV infected patients who had not previously been treated with DAAs were assigned randomly to an 8-week sofosbuvir/velpatasvir/voxilaprevir treatment arm or a 12-week sofosbuvir/velpatasvir treatment arm ( $n=941$, conventional or pegylated interferon experience $23 \%) .{ }^{43}$ In genotype 1a patients, the SVR after the 8-week sofosbuvir/velpatasvir/voxilaprevir or 12-week sofosbuvir/velpatasvir treatment were $92 \%$ (155/169) and 99\% (170/172), respectively. Among HCV genotype 1 ia infected patients without cirrhosis, the virologic relapse rates were $8 \%$ and $0 \%$ in the 8 -week sofosbuvir/velpatasvir/voxilaprevir and 12-week sofosbuvir/velpatasvir treatment groups, respectively. Among HCV genotype 1a infected patients with cirrhosis, the virologic relapse rates were $10 \%$ and $2 \%$ in the 8 -week sofosbuvir/velpatasvir/voxilaprevir and 12-week sofosbuvir/velpatasvir treatment groups, respectively. Therefore, among HCV genotype 1a infected patients, treatment responses after 8 weeks of sofosbuvir/velpatasvir/voxilaprevir were inferior to those after 12 weeks of sofosbuvir/velpatasvir. In addition, the SVR following treatment with sofosbuvir/velpatasvir/voxilaprevir for 8 weeks was $89 \%$ and $95 \%$ in HCV genotype 1a infected patients with and without baseline NS5A RASs, respectively.

\section{Retreatment of treatment-experienced genotype 1a patients}

\section{Ledipasvir/sofosbuvir}

In a phase 3, randomized, open-label study of previously treated HCV genotype 1 infected patients ( $n=440$, genotype 1a 79\%, cirrhosis 20\%, protease inhibitor experience 53\%), the SVR fol- lowing 12 weeks of ledipasvir/sofosbuvir, 12 weeks of ledipasvir/ sofosbuvir plus ribavirin, 24 weeks of ledipasvir/sofosbuvir, or 24 weeks of ledipasvir/sofosbuvir plus ribavirin were 94\% (102/109), 96\% (107/111), 99\% (108/109), and 99\% (110/111), respectively. ${ }^{44}$ Virologic relapse rates were 6\% (7/109), 4\% (4/111), 0\% (0/109), and $0 \%(0 / 111)$, respectively. Baseline viral load, age, IL28B genotype, and sub-genotype had no significant effect on treatment responses. In cirrhotic patients, the SVR following a 12-week ledipasvir/sofosbuvir treatment, 12-week ledipasvir/sofosbuvir plus ribavirin treatment, 24-week ledipasvir/sofosbuvir treatment, or 24-week ledipasvir/sofosbuvir plus ribavirin treatment were $86 \%$ $(19 / 22), 82 \%(18 / 22), 95 \%(21 / 22)$, and 100\% (22/22), respectively.

A post hoc analysis of data from seven clinical trials to evaluate the efficacy and safety of ledipasvir/sofosbuvir with and without ribavirin included 352 treatment-experienced HCV genotype 1 infected patients with compensated cirrhosis (genotype 1a 63\%, protease inhibitor experience $68 \%)^{57}$ The SVR following a 12 week ledipasvir/sofosbuvir treatment, 12-week ledipasvir/sofosbuvir plus ribavirin treatment, 24-week ledipasvir/sofosbuvir treatment, and 24-week ledipasvir/sofosbuvir plus ribavirin treatment were $90 \%$ (64/71), 96\% (152/159), 98\% (98/100), and 100\% $(22 / 22)$, respectively. The relatively lower SVR in treatment-experienced patients treated with ledipasvir/sofosbuvir for 12 weeks raised the question of whether those patients would benefit from adding ribavirin or extending treatment duration to 24 weeks.

Among treatment-experienced HCV genotype 1a patients, the overall SVR following ledipasvir/sofosbuvir with or without ribavirin treatment for 12 or 24 weeks was $76 \%(22 / 29)$ in patients with baseline ledipasvir-specific RASs (K24G/N/R, M28T/A/G, Q30L/T/E/G/H/K, L31F/I/M/V, P32L, S38F, H58D, A92T/K, Y93F/C/ $\mathrm{H} / \mathrm{N} / \mathrm{S})$ and $97 \%(409 / 420)$ in those without ledipasvir-specific RASs. ${ }^{20}$ In cirrhotic patients, the SVR was $77 \%(10 / 13)$ in patients with baseline ledipasvir-specific RASs and 98\% (193/196) in those without ledipasvir-specific RASs. That is, treatment-experienced HCV genotype 1a patients with baseline ledipasvir-specific RASs had a lower response irrespective of their cirrhosis status.

\section{Elbasvir/grazoprevir}

In a phase 3 study that assessed the efficacy and safety of a 12week treatment of elbasvir/grazoprevir in treatment-experienced HCV genotype 1a patients, the SVR was 92\% (55/60)..$^{49}$ The SVR was $93 \%$ (56/60) in patients treated with elbasvir/grazoprevir plus ribavirin for 12 weeks, 94\% (45/48) in those treated with elbasvir/grazoprevir for 16 weeks without ribavirin, and 100\% 
The Korean Association for the Study of the Liver (KASL) 2017 KASL clinical practice guidelines: Management of chronic hepatitis C

$(55 / 55)$ in those treated with elbasvir/grazoprevir plus ribavirin for 16 weeks. SVR did not differ significantly depending on the addition of ribavirin or the extension of the treatment duration. ${ }^{49}$ The SVR after 12 or 16 weeks of treatment with elbasvir/grazoprevir with and without ribavirin was 94\% (135/144) in patients with compensated cirrhosis (genotype 1a [ $n=77]$, genotype $1 b[n=50]$, genotype $4[n=17]$, genotype $6[n=3])$, which did not differ from that in patients without cirrhosis $\left(97 \%\right.$ [255/264]). ${ }^{49}$ The presence of baseline NS5A RASs was a predictive factor for treatment failure, and amino acid substitutions at positions M28, Q30, L31, and Y93 were associated with lower SVR. The SVR after a 12-week treatment with elbasvir/grazoprevir was $99 \%$ in HCV genotype 1a patients without NS5A RASs but only 68\% (21/31) in those with baseline NS5A RASs. The SVR after a 16- or 18-week treatment with elbasvir/grazoprevir plus ribavirin was $100 \%(6 / 6)$ in genotype 1a patients with baseline NS5A RASs. ${ }^{49}$ In another study, baseline NS5A RASs were detected in $21 \%$ of HCV genotype 1a patients, and the SVR after a 12-week treatment with elbasvir/ grazoprevir was significantly lower in patients with baseline NS5A RASs $\left(64 \%\right.$ [9/14]) than in those without them (96\% [52/54]). ${ }^{59}$ However, the SVR was $100 \%$ (8/8) in patients with NS5A RASs after a 16- or 18-week treatment with elbasvir/grazoprevir plus ribavirin. ${ }^{59}$ In a pooled analysis of phase 2 and 3 studies of DAAnaïve patients, the SVR after a 12-week treatment with elbasvir/ grazoprevir was 97\% (77/79) among PR-experienced HCV genotype 1a patients without NS5A RASs and 55\% (6/11) in those with NS5A RASs. The SVR after 16 or 18 weeks of treatment with elbasvir/grazoprevir plus ribavirin was $100 \%(6 / 6)$, even in those with NS5A RASs. ${ }^{24}$ Because the treatment response in patients with NS5A RASs is inferior, NS5A resistance testing is recommended for genotype 1a infected patients before elbasvir/grazoprevir treatment, and treatment duration should be extended to 16 weeks and ribavirin should be added if baseline NS5A RASs are identified.

\section{Ombitasvir/paritaprevir/ritonavir and dasabuvir}

Among treatment-experienced HCV genotype 1a patients without cirrhosis, the SVR after a 12-week treatment of ombitasvir/ paritaprevir/ritonavir plus dasabuvir with ribavirin was $96 \%$ (166/173). ${ }^{50,54}$ Among treatment-experienced HCV genotype 1 patients with compensated cirrhosis (genotype 1a 262, 1b 119), the SVR after 12 and 24 weeks of treatment with ombitasvir/paritaprevir/ritonavir plus dasabuvir with ribavirin was 90\% (110/122) and $97 \%$ (95/98), respectively, with no statistical differences between the groups $(P=0.09)$. However, the SVR was significantly lower in HCV genotype 1a infected patients (OR 0.12, $P=0.04)$ and genotype 1 patients who showed null response to prior PR therapy (OR 0.39, $P=0.04){ }^{27}$

\section{Daclatasvir and sofosbuvir}

In a phase 2 study of treatment-experienced HCV genotype 1 patients (genotype 1a 33, 1b 8, cirrhosis 9), the SVR after a 24week treatment of daclatasvir and sofosbuvir with and without ribavirin was 100\% (21/21) and 95\% (19/20), respectively. ${ }^{31}$ In a cohort study of 768 genotype 1 infected patients (genotype 1a $50 \%$, liver cirrhosis 73\%, CTP classification B or C decompensated cirrhosis 3\%, PR experience 41\%, 1st generation PI+PR experience $41 \%)$, the SVR after a 12 - or 24-week treatment of daclatasvir and sofosbuvir was 92\% (147/160) and 95\% (417/439), respectively, and the SVR after a 12- or 24-week treatment of daclatasvir and sofosbuvir with ribavirin was 94\% (32/34) and 99\% (133/135), respectively, without statistical difference among treatment groups. However, the SVR was lower in cirrhotic patients than in those without cirrhosis (88\% [105/119] vs. 95\% $[423 / 444], P=0.0054)^{32}$

\section{Glecaprevir/pibrentasvir}

In a phase 3 study that assessed the efficacy and safety of 8 or 12 weeks of treatment with glecaprevir $(300 \mathrm{mg}) /$ pibrentasvir (120 mg) in genotype 1 monoinfected or HIV/HCV co-infected patients without cirrhosis ( $n=703$, interferon-based treatment experience $28 \%$, sofosbuvir-based treatment experience $0.4 \%$ ), the SVR rates were $99 \%$ and $99.7 \%$, respectively. ${ }^{37}$

In a pooled analysis of phase 2 and 3 studies of patients with HCV genotypes 1-6 without cirrhosis (interferon-based treatment experience $23 \%$, sofosbuvir-based treatment experience $1 \%$ ), the SVR after an 8- or 12-week treatment with glecaprevir (300 mg) plus pibrentasvir (120 mg) was 99\% and 100\%, respectively, in HCV genotype 1 patients ( $n=788$, genotype 1a 44\%). ${ }^{38}$

In a phase 3 study that assessed the efficacy and safety of a 12week treatment with glecaprevir (300 mg)/pibrentasvir (120 mg) in patients infected with HCV genotype 1, 2, 4, 5, or 6 and compensated cirrhosis ( $n=146$, interferon-based treatment experience $17 \%$, sofosbuvir-based treatment experience $8 \%$ ), the SVR was $99 \%$ in HCV genotype 1a infected patients $(n=48) .{ }^{40}$

\section{Sofosbuvir/velpatasvir}

In a phase 3, double-blind, placebo-controlled study of untreated and previously treated patients infected with HCV genotype 1, $2,4,5$, or 6, including those with compensated cirrhosis, the SVR 
following a 12-week treatment with sofosbuvir (400 mg)/velpatasvir (100 mg) was 100\% (78/78) in previously treated HCV genotype 1a patients. ${ }^{42}$

\section{Sofosbuvir/velpatasvir/voxilaprevir}

In a phase 3 open-label trial, HCV infected patients who had not been previously treated with DAAs were randomly assigned to an 8-week treatment with sofosbuvir/velpatasvir/voxilaprevir or a 12-week treatment with sofosbuvir/velpatasvir ( $n=941$, conventional or pegylated interferon experience $23 \%$ ). ${ }^{43}$ Among genotype 1a patients, the SVR of the 8-week sofosbuvir/velpatasvir/ voxilaprevir and 12-week sofosbuvir/velpatasvir treatment groups were 92\% (155/169) and 99\% (170/172), respectively. In HCV genotype 1a patients without cirrhosis, the virologic relapse rates were $8 \%$ and $0 \%$ in the 8 -week sofosbuvir/velpatasvir/voxilaprevir and 12-week sofosbuvir/velpatasvir treatment groups, respectively. Among HCV genotype 1a patients with cirrhosis, virologic relapse rates were $10 \%$ and $2 \%$ in the 8 -week sofosbuvir/velpatasvir/voxilaprevir and 12-week sofosbuvir/velpatasvir treatment groups, respectively. Therefore, among HCV genotype 1a patients, treatment responses after 8 weeks of sofosbuvir/velpatasvir/voxilaprevir treatment were inferior to those after 12 weeks of sofosbuvir/velpatasvir treatment. In addition, the SVR after the 8-week treatment with sofosbuvir/velpatasvir/voxilaprevir was $89 \%$ and $95 \%$, respectively, in HCV genotype 1a patients with and without baseline NS5A RASs.

\section{[Recommendations] (Table 7)}

Initial treatment of chronic hepatitis $\mathrm{C}$ or compensated cirrhosis patients with HCV genotype 1a infection

1. Ledipasvir/sofosbuvir should be administered for 12 weeks (A1). Treatment could be shortened to 8 weeks in patients without liver cirrhosis and without HIV co-infection whose baseline HCV RNA level is less than $6,000,000 \mathrm{IU} / \mathrm{mL}$ (B1)

2. Elbasvir/grazoprevir should be administered for 12 weeks to patients without NS5A RASs (A1).

Elbasvir/grazoprevir and ribavirin could be administered for 16 weeks to patients with NS5A RASs (B1).

3. Ombitasvir/paritaprevir/ritonavir plus dasabuvir and ribavirin should be administered for 12 weeks to patients without liver cirrhosis (A1). Ombitasvir/paritaprevir/ritonavir plus dasabuvir and ribavirin should be administered for 24 weeks to patients with compensated cirrhosis (A1).

4. Daclatasvir and sofosbuvir should be administered for 12 weeks to patients without liver cirrhosis (A1)

Daclatasvir, sofosbuvir, and ribavirin could be administered for 12 weeks to patients with compensated cirrhosis, or they could receive daclatasvir and sofosbuvir for 24 weeks (B1).
5. Glecaprevir/pibrentasvir should be administered for 8 weeks to patients without liver cirrhosis (A1)

Glecaprevir/pibrentasvir should be administered for 12 weeks to patients with compensated cirrhosis (A1).

6. Sofosbuvir/velpatasvir should be administered for 12 weeks (A1).

Retreatment of treatment-experienced chronic hepatitis $C$ or compensated cirrhosis patients with HCV genotype 1a infection 1. Ledipasvir/sofosbuvir and ribavirin should be administered for 12 weeks, or ledipasvir/sofosbuvir should be administered for 24 weeks (A1).

2. Elbasvir/grazoprevir should be administered for 12 weeks to patients without NS5A RASs (A1)

Elbasvir/grazoprevir and ribavirin could be administered for 16 weeks to patients with NS5A RASs (B1).

3. Ombitasvir/paritaprevir/ritonavir plus dasabuvir and ribavirin should be administered for 12 weeks to patients without liver cirrhosis (A1).

Ombitasvir/paritaprevir/ritonavir plus dasabuvir and ribavirin should be administered for 24 weeks to patients with compensated cirrhosis (A1).

4. Daclatasvir and sofosbuvir could be administered for 12 weeks to patients without liver cirrhosis (B1).

Daclatasvir, sofosbuvir, and ribavirin could be administered for 12 weeks to patients with compensated cirrhosis, or they could receive daclatasvir and sofosbuvir for 24 weeks (B1).

5. Glecaprevir/pibrentasvir should be administered for 8 weeks to patients without liver cirrhosis (A1).

Glecaprevir/pibrentasvir should be administered for 12 weeks to patients with compensated cirrhosis (A1).

6. Sofosbuvir/velpatasvir should be administered for 12 weeks (A1).

\title{
TREATMENT OF CHRONIC HEPATITIS C OR COMPENSATED CIRRHOSIS PATIENTS WITH HCV GENOTYPE 2 INFECTION
}

\section{Initial treatment of genotype 2 patients}

\author{
Sofosbuvir and ribavirin \\ According to a study comparing 12 weeks of sofosbuvir and rib- \\ avirin with 24 weeks of peginterferon alpha and ribavirin in treat- \\ ment-naïve patients with chronic hepatitis C genotype 2 , the SVR \\ were $97 \%(68 / 70)$ and $78 \%(52 / 67)$, respectively. The SVR fol- \\ lowing 12 weeks of sofosbuvir and ribavirin in patients with and \\ without liver cirrhosis was $91 \%$ (10/11) and 98\% (58/59), respec- \\ tively. ${ }^{62}$ \\ In a phase 3 study of patients ineligible for peginterferon thera- \\ py (including $8 \%$ of enrolled patients who discontinued therapy \\ due to an adverse event), the SVR following 12 weeks of sofosbu- \\ vir and ribavirin therapy was 93\% (101/109). The SVR in patients
}


The Korean Association for the Study of the Liver (KASL) 2017 KASL clinical practice guidelines: Management of chronic hepatitis C

with and without liver cirrhosis was $94 \%(16 / 17)$ and $92 \%$ (85/92), respectively. ${ }^{62}$

In a phase 3 study of Korean and Taiwanese patients, the SVR following 12 weeks of sofosbuvir and ribavirin treatment was 96\% (101/105), and all 6 patients with liver cirrhosis showed an SVR. ${ }^{63}$ In a Japanese study, the SVR rate following 12 weeks of sofosbuvir and ribavirin was $98 \%$ (88/90), and all 8 patients with liver cirrhosis showed an SVR. ${ }^{64}$

In a phase 3 study of 12 weeks of sofosbuvir and ribavirin therapy, the SVR in treatment-naive patients was 97\% (31/32), and the SVR in patients with and without liver cirrhosis were $100 \%$ (2/2) and $97 \%(29 / 30)$, respectively. ${ }^{65}$

Studies on the optimal treatment duration of sofosbuvir and ribavirin therapy are very limited in treatment-naïve patients with chronic HCV infection and liver cirrhosis.

In a Korean real life study, the SVR following 12 weeks of sofosbuvir and ribavirin was 98\% (177/181) in treatment-naïve patients without liver cirrhosis. The SVR after 16 weeks of sofosbuvir and ribavirin therapy was $96 \%$ (50/52) in patients with liver cirrhosis, although it is not possible to distinguish between treatment-naive and experienced patients in that analysis. ${ }^{66}$

In a real life study of sofosbuvir and ribavirin therapy among treatment-naïve and experienced patients, the SVR of treatmentnaive patients with or without liver cirrhosis was 72\% (23/32) and $92 \%$ (159/173), respectively, after 12 weeks of therapy and 100\% (7/7) and $91 \%$ (10/11), respectively, after 16 weeks of therapy. ${ }^{67}$

Another real life study (treatment naïve $56 \%$, liver cirrhosis $58 \%$ ) included patients with advanced fibrosis, who received 12 weeks of sofosbuvir and ribavirin $(n=123)$, and patients with liver cirrhosis, who received 16 or 20 weeks of sofosbuvir and ribavirin, ( $n=168$, CTP class A 89\%). The overall SVR in treatment-naïve patients was $98 \%$. Although it is not possible to distinguish be- tween treatment-naive and experienced patients, the SVR following 16 and 20 weeks of treatment in patients with cirrhosis were $95 \%(86 / 91)$ and $91 \%(75 / 82)$, respectively. ${ }^{68}$

\section{Daclatasvir and sofosbuvir}

Only a few studies have considered daclatasvir and sofosbuvir combination therapy in patients with HCV genotype 2 .

In a phase 2 study of daclatasvir and sofosbuvir therapy for treatment-naive patients, the SVR was $92 \%$ (24/26). That study conducted daclatasvir and sofosbuvir therapy with and without ribavirin for 23 weeks (sofosbuvir monotherapy for 1 week followed by 23 consecutive weeks of daclatasvir and sofosbuvir with or without ribavirin) or 24 weeks, and $40 \%$ of the included patients had liver cirrhosis. ${ }^{31}$

In a study of daclatasvir and sofosbuvir for 8 or 12 weeks in patients with HIV/HCV coinfection, the SVR in treatment-naive patients without liver cirrhosis was 83\% (5/6) and 100\% (11/11), respectively. ${ }^{30}$

Based on a sub-group analysis of genotype 2 patients from a real life study of genotypes $1-4$ (total $n=2,612$, treatment naive $47 \%), 12$ weeks of daclatasvir and sofosbuvir combination therapy yielded $100 \%$ SVR in all patients with $(n=29)$ and without $(n=17)$ cirrhosis, although it is not possible to distinguish between treatment-naive and experienced patients in that subpopulation. ${ }^{69}$

\section{Glecaprevir/pibrentasvir}

According to integrated analyses of seven phase 2 and 3 studies of glecaprevir/pibrentasvir treatment for 8 and 12 weeks in patients with genotypes 1-6 without cirrhosis (treatment-naive $79 \%$ and $74 \%$, respectively), the SVR of patients with genotype 2 was 99\% (193/195) and 100\% (232/232), respectively. ${ }^{38}$

In a phase 3 study (treatment naïve 75\%) of a 12-week regimen

Table 7. Treatment of chronic hepatitis C or compensated cirrhosis patients with HCV genotype la infection

\begin{tabular}{|c|c|c|c|c|}
\hline & \multicolumn{2}{|c|}{ Treatment naive } & \multicolumn{2}{|c|}{ PR experienced } \\
\hline & Chronic hepatitis & Compensated cirrhosis & Chronic hepatitis & Compensated cirrhosis \\
\hline Ledipasvir/sofosbuvir & $12 w k\left(8 w k^{*}\right)$ & $12 w k$ & $12 w k+R / 24 w k$ & $12 w k+R / 24 w k$ \\
\hline Elbasvir/grazoprevir & $\begin{array}{c}12 w k \\
\left(16 w k+R \text { if } R A S^{\dagger}+\right)\end{array}$ & $\begin{array}{c}12 w k \\
\left(16 w k+R \text { if } \operatorname{RAS}^{\dagger}+\right)\end{array}$ & $\begin{array}{c}12 \text { wk } \\
\left(16 w k+\text { R } \text { RAS }^{\dagger}+\right)\end{array}$ & $\begin{array}{c}12 w k \\
\left(16 w k+R \text { if } R A S^{\dagger}+\right)\end{array}$ \\
\hline Ombitasvir/paritaprevir/ritonavir+dasabuvir & $12 w k+R$ & $24 w k+R$ & $12 w k+R$ & $24 w k+R$ \\
\hline Daclatasvir+sofosbuvir & $12 w k$ & $24 w k / 12 w k+R$ & $12 w k$ & $24 w k / 12 w k+R$ \\
\hline Glecaprevir/pibrentasvir & $8 w k$ & $12 w k$ & $8 w k$ & $12 w k$ \\
\hline Sofosbuvir/velpatasvir & $12 w k$ & $12 w k$ & $12 w k$ & $12 w k$ \\
\hline
\end{tabular}

HCV, hepatitis C virus; PR, pegylated interferon alpha+ribavirin; wk, weeks; R, weight-based ribavirin; RAS, resistance-associated substitution. *Without liver cirrhosis, without human immunodeficiency virus co-infection, and HCV RNA level of less than 6,000,000 IU/mL; ${ }^{\dagger} \mathrm{NS} 5 \mathrm{~A}$ RAS. 
of glecaprevir/pibrentasvir given to cirrhotic patients with genotypes $1,2,4,5$, or 6 , the SVR in patients with genotype $2 \mathrm{HCV}$ infection was $100 \%(31 / 31){ }^{40}$

\section{Sofosbuvir/velpatasvir}

In a phase 3 study comparing 12 weeks of sofosbuvir/velpatasvir with placebo in patients with genotype $1,2,4,5$, or 6 infection (treatment naive $68 \%$, liver cirrhosis $19 \%$ ), the SVR of patients with genotype 2 who received sofosbuvir/velpatasvir therapy was $100 \%(104 / 104){ }^{42}$

According to a phase 3 study comparing treatment with sofosbuvir/velpatasvir for 12 weeks and sofosbuvir and ribavirin for 12 weeks (treatment naive $85 \%$, liver cirrhosis $14 \%$ ), the SVR were $99 \%(133 / 134)$ and $94 \%(124 / 132)$, respectively $(P=0.02){ }^{70}$

An integrated study of 12 weeks of sofosbuvir/velpatasvir showed an SVR of 99\% (193/194) among treatment-naïve patients with genotype 2. The SVR of patients with liver cirrhosis was 100\% (29/29), although it is not possible to distinguish between treatment-naive and experienced patients in that subpopulation. $^{71}$

\section{Sofosbuvir/velpatasvir/voxilaprevir}

In a phase 3 study comparing 8 weeks of sofosbuvir/velpatasvir/ voxilaprevir with 12 weeks of sofosbuvir/velpatasvir in patients with genotypes 1-6 (treatment naïve $77 \%$, liver cirrhosis 18\%), the SVR of patients with genotype 2 was $97 \%(61 / 63)$ and $100 \%$ (53/53), respectively. ${ }^{43}$

\section{Peginterferon alpha and ribavirin}

The SVR of peginterferon alpha and ribavirin therapy among Korean patients with genotype 2 is reported to be $80 \%$ or more. ${ }^{72,73}$ Peginterferon-a 2 a should be injected at a subcutaneous dose of $180 \mu \mathrm{g}$ once a week, regardless of patient body weight, and peginterferon- $\mathrm{a} 2 \mathrm{~b}$ is to be injected at a dose of 1.5 $\mu \mathrm{g} / \mathrm{kg} /$ week. Ribavirin should be administered as an $800 \mathrm{mg}$ fixed dose, regardless of body weight or type of peginterferon. ${ }^{74-79}$

\section{Retreatment of treatment-experienced patients}

\section{Sofosbuvir and ribavirin}

In one phase 3 study of sofosbuvir and ribavirin for 12 weeks, the SVR of treatment-experienced patients was $90 \%$ (37/41): $78 \%(7 / 9)$ and $94 \%(30 / 32)$ with and without liver cirrhosis, respectively. ${ }^{65}$

In a Korean real life study, the SVR following 12 weeks of sofos- buvir and ribavirin was 97\% (32/33) among treatment-experienced patients without liver cirrhosis. The SVR following 16 weeks of sofosbuvir and ribavirin was 96\% (50/52) among patients with liver cirrhosis, although it is not possible to distinguish between treatment-naive and experienced patients in that subpopulation. ${ }^{66}$

In a phase 3 study of sofosbuvir and ribavirin therapy for treatment-experienced patients, the SVR with and without liver cirrhosis was $87 \%$ (26/30) and $88 \%(42 / 48)$, respectively, following 12 weeks of therapy and $77 \%(13 / 17)$ and $100 \%(3 / 3)$, respectively, following 16 weeks of therapy. ${ }^{62}$

In a real life study of sofosbuvir and ribavirin for treatment-naïve and experienced patients, the SVR of treatment-experienced patients with and without liver cirrhosis was 72\% (23/32) and 92\% (159/173), respectively, following 12 weeks of therapy and 100\% (7/7) and 91\% (10/11), respectively, following 16 weeks of therapy. ${ }^{67}$

In a study comparing 12 weeks and 16 weeks of sofosbuvir and ribavirin therapy, the SVR of treatment experienced patients with liver cirrhosis was $87 \%$ (13/15) and 100\% (17/17), respectively. ${ }^{80}$

In phase 3 study of Korean and Taiwanese patients, the SVR in treatment-experienced patients after 12 weeks of sofosbuvir and ribavirin therapy was $100 \%$ (24/24, including 7 liver cirrhosis patients)..$^{63}$ In a Japanese study, the SVR following 12 weeks of sofosbuvir and ribavirin therapy was 95\% (60/63, including SVR of $8 / 9$ with liver cirrhosis)..$^{64}$

In a real life study of 16 or 20 weeks of sofosbuvir and ribavirin therapy for patients with liver cirrhosis (treatment-experienced $51 \%)$, the SVR in patients with previous non-response and relapse was $91 \%$ and $100 \%$, respectively. Although it is not possible to distinguish between treatment-naive and experienced patients in this analysis, the SVR following 16 and 20 weeks of treatment in patients with cirrhosis was 95\% (86/91) and 91\% (75/82), respectively. ${ }^{68}$

\section{Daclatasvir and sofosbuvir}

In a study of 12 weeks of daclatasvir and sofosbuvir for patients with HIV/HCV co-infection, 2 treatment-experienced patients had an SVR, including one patient with liver cirrhosis. ${ }^{30}$

Based on a sub-group analysis of patients with HCV genotype 2 in a real life study (genotypes $1-4, n=2,612$, treatment experience $53 \%$ ), daclatasvir and sofosbuvir combination therapy for 12 weeks yielded 100\% SVR in all 17 patients without cirrhosis and all 29 patients with cirrhosis, although it is not possible to distinguish between treatment-naive and experienced patients in that 
The Korean Association for the Study of the Liver (KASL) 2017 KASL clinical practice guidelines: Management of chronic hepatitis C

analysis. $^{69}$

\section{Glecaprevir/pibrentasvir}

According to an integrated analysis of seven phase 2 and 3 studies of glecaprevir/pibrentasvir for 8 or 12 weeks in patients with genotypes 1-6 without cirrhosis (treatment-experienced $21 \%$ and $26 \%$, respectively), the SVR in patients with genotype 2 was $99 \%(193 / 195)$ and $100 \%$ (232/232), respectively. ${ }^{38}$

In a phase 3 study of 12 weeks of glecaprevir/pibrentasvir for cirrhotic patients with genotypes 1, 2, 4, 5, or 6 (treatment-experienced $25 \%$ ), the SVR of patients with genotype 2 was $100 \%$ $(31 / 31)^{40}$

\section{Sofosbuvir/velpatasvir}

In a phase 3 study comparing a 12-week course of sofosbuvir/ velpatasvir with placebo in patients with genotypes $1,2,4,5$, or 6 (treatment experienced 32\%, liver cirrhosis 19\%), the SVR of patients with genotype 2 was $100 \%(104 / 104){ }^{42}$

According to a phase 3 study comparing 12 weeks of treatment with sofosbuvir/velpatasvir with 12 weeks of sofosbuvir and ribavirin therapy (treatment experienced 15\%, liver cirrhosis 14\%), the SVR were 99\% (133/134) and 94\% (124/132), respectively. ${ }^{70}$

In an integrated study of sofosbuvir/velpatasvir for 12 weeks, the SVR was 100\% (44/44) among treatment-experienced patients with genotype 2 . The SVR of patients with liver cirrhosis was also $100 \%(29 / 29)^{71}$

\section{Sofosbuvir/velpatasvir/voxilaprevir}

In a phase 3 study comparing 8 weeks of sofosbuvir/velpatasvir/ voxilaprevir with 12 weeks of sofosbuvir/velpatasvir in patients with genotypes 1-6 (treatment experienced 23\%, liver cirrhosis $18 \%)$, the SVR of patients with genotype 2 was $97 \%$ (61/63) and $100 \%(53 / 53)$, respectively. ${ }^{43}$

\section{[Recommendations] (Table 8)}

Initial treatment of chronic hepatitis $\mathrm{C}$ or compensated cirrhosis patients with HCV genotype 2 infection

1. Sofosbuvir and ribavirin should be administered for 12 weeks to patients without liver cirrhosis (A1).

Sofosbuvir and ribavirin could be administered for 16 weeks to patients with liver cirrhosis (B1).

2. Daclatasvir and sofosbuvir could be administered for 12 weeks (B1).

3. Glecaprevir/pibrentasvir should be administered for 8 weeks to patients without liver cirrhosis (A1)

Glecaprevir/pibrentasvir should be administered for 12 weeks to patients with liver cirrhosis (A1).

4. Sofosbuvir/velpatasvir should be administered for 12 weeks (A1).

5. Peginterferon alpha and ribavirin could be administered for 24 weeks (A2).

Retreatment of treatment-experienced chronic hepatitis $\mathrm{C}$ or compensated cirrhosis patients with HCV genotype 2 infection 1. Sofosbuvir and ribavirin should be administered for 12 weeks to patients without liver cirrhosis (A1)

Sofosbuvir and ribavirin could be administered for 16-24 weeks to patients with liver cirrhosis (B1).

2. Daclatasvir and sofosbuvir could be administered for 12 weeks (B1).

3. Glecaprevir/pibrentasvir should be administered for 8 weeks to patients without liver cirrhosis (A1). Glecaprevir/pibrentasvir should be administered for 12 weeks to patients with liver cirrhosis (A1)

4. Sofosbuvir/velpatasvir should be administered for 12 weeks (A1).

\section{TREATMENT OF CHRONIC HEPATITIS C OR COMPENSATED CIRRHOSIS PATIENTS WITH HCV GENOTYPE 3 INFECTION}

\section{Initial treatment of genotype 3 patients}

\section{Daclatasvir and sofosbuvir}

Although daclatasvir was more potent than ledipasvir for viral

Table 8. Treatment of chronic hepatitis $C$ or compensated cirrhosis patients with hepatitis $C$ virus genotype 2 infection

\begin{tabular}{|c|c|c|c|c|}
\hline & \multicolumn{2}{|c|}{ Treatment naive } & \multicolumn{2}{|c|}{ PR experienced } \\
\hline & Chronic hepatitis & Compensated cirrhosis & Chronic hepatitis & Compensated cirrhosis \\
\hline Sofosbuvir+R & $12 w k$ & $16 w k$ & $12 w k$ & $16-24 w k$ \\
\hline Daclatasvir+sofosbuvir & $12 w k$ & $12 w k$ & $12 w k$ & $12 w k$ \\
\hline Glecaprevir/pibrentasvir & $8 w k$ & $12 w k$ & $8 w k$ & 12 wk \\
\hline Sofosbuvir/velpatasvir & $12 w k$ & $12 w k$ & $12 w k$ & $12 w k$ \\
\hline$P R$ & $24 w k$ & $24 w k$ & & \\
\hline
\end{tabular}

PR, pegylated interferon alpha+ribavirin 800 mg; R, weight-based ribavirin; wk, weeks. 
suppression in a pharmacodynamic study of HCV genotype 3. 81,82 studies on daclatasvir and sofosbuvir combination therapy are very limited.

In a phase 3 study of 12 weeks of daclatasvir and sofosbuvir, the overall SVR for treatment-naïve patients was 90\% (91/101), with an SVR of 58\% (11/19) and 97\% (73/75) in patients with and without liver cirrhosis, respectively. ${ }^{83}$

Based on a sub-group analysis of patients with genotype 3 in a real life study of patients with genotype $1-4$ infection $(n=2,612$, treatment naïve 47\%), 12 weeks of daclatasvir and sofosbuvir showed an SVR of 95\% (18/19) in patients without cirrhosis, and 12 weeks of daclatasvir, sofosbuvir, and ribavirin showed an SVR of $92 \%(121 / 131)$ in patients with liver cirrhosis, although it is not possible to distinguish between treatment-naive and -experienced patients. $^{69}$

In a study evaluating the efficacy of 12 and 16 weeks of a daclatasvir and sofosbuvir combination regimen in patients with genotype 3 (treatment naïve $26 \%$, liver cirrhosis $72 \%$ ), the overall SVR was $80 \%$ (21/24) and 92\% (24/26), respectively; the corresponding SVR in treatment-naïve patients were $83 \%(5 / 6)$ and $100 \%(7 / 7)$, respectively. ${ }^{84}$

In a phase 2 study of 24 weeks of daclatasvir or sofosbuvir with or without ribavirin, the SVR was $89 \%(16 / 18){ }^{31}$

In a study of 12 and 24 weeks of daclatasvir and sofosbuvir therapy in patients with liver cirrhosis (combined use of ribavirin in $86 \%$ and $78 \%$ of patients, respectively), the SVR was $92 \%$ (34/37) and 95\% (89/94), respectively, with an overall SVR of $93 \%(68 / 73)$ in treatment-naïve patients. ${ }^{85}$

In a real life study of 24 weeks of daclatasvir and sofosbuvir with or without ribavirin (27\% with liver cirrhosis), the SVR in treatment naïve patients was $100 \%(12 / 12)$ and $92 \%$ (23/25), respectively, though it is not possible to distinguish the presence or absence of liver cirrhosis in that analysis. ${ }^{86}$

The effects of RASs on daclatasvir and sofosbuvir combination therapy were tested in a phase 3 study of 12 weeks of daclatasvir and sofosbuvir. Although the researchers did not complete a subanalysis between treatment-naïve and -experienced patients, the SVR in RASs-positive patients with and without liver cirrhosis was $30 \%(3 / 10)$ and $83 \%(15 / 18)$, respectively. ${ }^{83}$

\section{Glecaprevir/pibrentasvir}

In a phase 3 study comparing 8 and 12 weeks of glecaprevir/pibrentasvir and 12 weeks of daclatasvir and sofosbuvir in treatment-naïve patients without liver cirrhosis, the SVR was 95\% (149/157), 95\% (222/233), and 97\% (111/115), respectively. ${ }^{87}$
According to an integrated analysis of seven phase 2 and 3 studies of glecaprevir/pibrentasvir for 8 or 12 weeks in patients with genotypes 1-6 without cirrhosis (all patients with genotype 3 were treatment-naive), the SVR of patients with genotype 3 was 97\% (177/183) and 98\% (258/262), respectively. ${ }^{38}$

In a phase 2 study comparing 12 weeks of combination therapy with glecaprevir (300 mg) and pibrentasvir (120 mg) with glecaprevir (300 mg), pibrentasvir (120 mg), and ribavirin (800 mg), the SVR in treatment-naïve patients were 100\% (24/24) and 100\% (24/24), respectively. ${ }^{39}$

\section{Sofosbuvir/velpatasvir}

In a phase 3 study comparing 12 weeks of sofosbuvir/velpatasvir with 12 weeks of sofosbuvir and ribavirin, the SVR in treatment-naïve patients were 97\% (200/206) and 87\% (174/201), respectively. ${ }^{70}$ Although the SVR with sofosbuvir and ribavirin combination therapy for 12 weeks differed significantly between patients with and without liver cirrhosis (73\% [33/45] and 90\% [141/156], respectively), the SVR of sofosbuvir/velpatasvir for 12 weeks was similar between patients with and without liver cirrhosis (93\% [40/43] and 98\% [160/163], respectively). Although the researchers provide no detailed data according to treatment experience, the SVR in patients with and without an NS5A RAS (16\%) was $88 \%(38 / 43)$ and $97 \%(225 / 231)$, respectively. Moreover, the SVR in patients with $\mathrm{Y} 93 \mathrm{H}$ was $84 \%$ (21/25).

Given the current situation in Korea, where genotype 3-specific RAS testing is unavailable, the combined use of ribavirin is highly recommended in patients with liver cirrhosis.

\section{Sofosbuvir/velpatasvir/voxilaprevir}

A phase 3 study compared 8 weeks of sofosbuvir/velpatasvir/ voxilaprevir with 12 weeks of sofosbuvir/velpatasvir in patients with genotypes 1-6 (among patients with genotype 3, treatment naive $77 \%$, none of liver cirrhosis). The SVR of patients with genotype 3 was 99\% (91/92) and 97\% (86/89), respectively. ${ }^{43}$

In a phase 3 study comparing 8 weeks of sofosbuvir/velpatasvir/ voxilaprevir and 12 weeks of sofosbuvir/velpatasvir for cirrhotic patients with genotype 3 (69\% treatment naïve), the SVR was 96\% (106/110) and 96\% (105/109), respectively.

\section{Sofosbuvir, peginterferon alpha, and ribavirin}

In a phase 2 study of 12 weeks of sofosbuvir, pegylated interferon, and ribavirin in treatment-naïve patients without liver cirrhosis, the SVR was $92-100 \%{ }^{88,89}$

In a phase 3 study of 12 weeks of sofosbuvir, pegylated inter- 
The Korean Association for the Study of the Liver (KASL) 2017 KASL clinical practice guidelines: Management of chronic hepatitis C

feron, and ribavirin, the SVR in treatment-naïve patients was $95 \%$ (89/94), and that in patients with and without liver cirrhosis was $91 \%(21 / 23)$ and $96 \%(68 / 71)$, respectively. ${ }^{80}$

\section{Peginterferon alpha and ribavirin}

Genotype 3 chronic HCV infection is very rare in Korea, and information about the efficacy of peginterferon therapy is lacking. Studies from Western countries show that the SVR following a 24week combination therapy of peginterferon alpha and ribavirin for genotype $3 \mathrm{HCV}$ infection was $60-70 \%, 10-20 \%$ lower than that for genotype 2 chronic hepatitis $C^{90-92}$

Peginterferon-a 2a should be injected at a subcutaneous dose of $180 \mu \mathrm{g}$ once a week, regardless of patient body weight, and peginterferon-a $2 \mathrm{~b}$ should be injected at a dose of $1.5 \mu \mathrm{g} / \mathrm{kg} /$ week. Ribavirin should be administered at a fixed dose of 800 $\mathrm{mg}$, regardless of body weight or type of peginterferon. ${ }^{74,76}$

\section{Retreatment of treatment-experienced patients}

\section{Daclatasvir and sofosbuvir}

In a phase 3 study of 12 weeks of daclatasvir and sofosbuvir, the overall SVR was 86\% (44/51) for treatment-experienced patients, with an SVR of $69 \%(9 / 13)$ and $94 \%$ (32/34) in patients with and without liver cirrhosis, respectively. ${ }^{83}$

Based on a sub-group analysis of patients with genotype 3 in a real life study of patients with genotypes $1-4$ (total $n=2,612$, treatment experience 53\%), 12 weeks of daclatasvir and sofosbuvir produced an SVR of $95 \%$ (18/19) in patients without cirrhosis, and 12 weeks of daclatasvir, sofosbuvir, and ribavirin produced an SVR of $92 \%(121 / 131)$ in patients with liver cirrhosis, although it is not possible to distinguish between treatment-naive and -experienced patients in that analysis. ${ }^{69}$

In a study of 12 and 16 weeks of daclatasvir and sofosbuvir combination therapy in patients with genotype 3 (treatment experienced $74 \%$, liver cirrhosis $72 \%$ ), the SVR rate in treatment-experienced patients was $88 \%(14 / 16)$ and $86 \%$ (12/14), respectively. The SVR in patients with and without liver cirrhosis, including treatment-naïve patients, was 83\% (15/18) and 89\% (16/18), respectively. ${ }^{84}$

In a real life study of 12 and 24 weeks of daclatasvir and sofosbuvir therapy (ribavirin combination in $86 \%$ and $78 \%$, respectively) among patients with liver cirrhosis, the overall SVR was $92 \%$ $(34 / 37)$ and $95 \%$ (89/94), respectively, with an SVR of $94 \%$ (55/58) among treatment-experienced patients. ${ }^{85}$

In another real life study of 24 weeks of daclatasvir and sofos- buvir combination therapy with and without ribavirin (liver cirrhosis 27\%), the SVR in treatment-experienced patients was $84 \%$ (21/25) and $84 \%$ (26/31), respectively. ${ }^{86}$ The SVR rate with a combination regimen of daclatasvir, sofosbuvir, and ribavirin in patients with liver cirrhosis, including treatment-naïve patients, was $88 \%(29 / 33)$.

An additional real life study conducted compassionate daclatasvir and sofosbuvir therapy for 12 or 24 weeks with and without ribavirin (treatment experienced $72 \%$, liver cirrhosis $77 \%$ ) in patients with liver cirrhosis or pre/post-liver transplantation. The SVR was $73 \%(48 / 66), 60 \%$ (6/10), 89\% (174/196), and 82\% (50/61) following daclatasvir and sofosbuvir for 12 weeks, daclatasvir, sofosbuvir, and ribavirin for 12 weeks, daclatasvir and sofosbuvir for 24 weeks, and daclatasvir, sofosbuvir, and ribavirin for 24 weeks, respectively. ${ }^{93}$

\section{Elbasvir/grazoprevir and sofosbuvir}

In a phase 2 study comparing 12 weeks of elbasvir/grazoprevir and sofosbuvir, 12 weeks of elbasvir/grazoprevir, sofosbuvir, and ribavirin, and 16 weeks of elbasvir/grazoprevir and sofosbuvir in cirrhotic patients with genotype $3 \mathrm{HCV}$, the SVR were 100\% (17/17), $94 \%$ (17/18), and 94\% (17/18), respectively. ${ }^{94}$

\section{Glecaprevir/pibrentasvir}

In a phase 2 study of 12 and 16 weeks of glecaprevir/pibrentasvir in patients without liver cirrhosis and 16 weeks of glecaprevir/ pibrentasvir in treatment-experienced patients with liver cirrhosis (peginterferon and ribavirin experienced 54\%, sofosbuvir and ribavirin experienced with or without peginterferon $46 \%$ ), the SVR were $91 \%$ (20/22), $96 \%$ (21/22), and 96\% (45/47), respectively. ${ }^{95}$

In a phase 2 study comparing 12 weeks of combination therapy of glecaprevir (300 mg) and pibrentasvir (120 mg) with 12 weeks of glecaprevir (300 mg), pibrentasvir (120 mg), and ribavirin (800 $\mathrm{mg})$, the SVR in treatment-experienced patients was $75 \%(3 / 4)$ and $100 \%(3 / 3)$, respectively. ${ }^{39}$

\section{Sofosbuvir/velpatasvir}

In a phase 3 study comparing 12 weeks of sofosbuvir/velpatasvir and 12 weeks of sofosbuvir and ribavirin, the SVR of treatment-experienced patients was 90\% (64/71) and 64\% (44/69), respectively $(P<0.001){ }^{70}$ The SVR following 12 weeks of sofosbuvir and ribavirin in patients with and without liver cirrhosis was $58 \%(22 / 38)$ and $71 \%(22 / 31)$, respectively. The SVR rates from 12 weeks of sofosbuvir/velpatasvir in patients with and without liver cirrhosis were 89\% (33/37) and 91\% (31/34), respectively. 


\section{Sofosbuvir/velpatasvir/voxilaprevir}

In a phase 3 study comparing 8 weeks of sofosbuvir/velpatasvir/ voxilaprevir with 12 weeks of sofosbuvir/velpatasvir in patients with genotypes 1-6 (among patients with genotype 3, treatment experienced 23\%, none of liver cirrhosis), the SVR of patients with genotype 3 was $99 \%$ (91/92) and 97\% (86/89), respectively. ${ }^{43}$

In a phase 3 study comparing 8 weeks of sofosbuvir/velpatasvir/ voxilaprevir and 12 weeks of sofosbuvir/velpatasvir in cirrhotic patients with genotype 3 (treatment experience 31\%), the SVR was 96\% (106/110) and 96\% (105/109), respectively.

\section{Sofosbuvir, peginterferon alpha, and ribavirin}

In a phase 3 study of 12 weeks of sofosbuvir, pegylated interferon, and ribavirin, the SVR in treatment-experienced patients was $93 \%(166 / 181)$. The SVR in patients with and without liver cirrhosis was $86 \%$ (30/35) and $94 \%$ (49/52), respectively. ${ }^{80}$

\footnotetext{
[Recommendations] (Table 9)

Initial treatment of chronic hepatitis $\mathrm{C}$ or compensated cirrhosis patients with HCV genotype 3 infection

1. Daclatasvir and sofosbuvir could be administered for 12 weeks to patients without liver cirrhosis (B1).

Daclatasvir, sofosbuvir, and ribavirin could be administered for 24 weeks to patients with liver cirrhosis (B1).

2. Glecaprevir/pibrentasvir should be administered for 8 weeks to patients without liver cirrhosis (A1).

Glecaprevir/pibrentasvir should be administered for 12 weeks to patients with liver cirrhosis (B1).

3. Sofosbuvir/velpatasvir should be administered for 12 weeks to patients without liver cirrhosis (A1).

Sofosbuvir/velpatasvir and ribavirin should be administered for 12 weeks to patients with liver cirrhosis (A1).

4. Sofosbuvir/velpatasvir/voxilaprevir should be administered for 8 weeks to patients with liver cirrhosis (A1).

5. Peginterferon alpha and ribavirin could be administered for 24 weeks (A2).
}

Retreatment of treatment-experienced chronic hepatitis $C$ or compensated cirrhosis patients with HCV genotype 3 infection

1. Daclatasvir, sofosbuvir, and ribavirin could be administered for 12 weeks to patients without liver cirrhosis (B1).

Daclatasvir, sofosbuvir, and ribavirin could be administered for 24 weeks to patients with liver cirrhosis (B1).

2. Elbasvir/grazoprevir and sofosbuvir could be administered for 12 weeks to patients with liver cirrhosis (B1).

3. Glecaprevir/pibrentasvir could be administered for 16 weeks (B1).

4. Sofosbuvir/velpatasvir and ribavirin should be administered for 12 weeks (A1)

5. Sofosbuvir/velpatasvir/voxilaprevir should be administered for 8 weeks to patients with liver cirrhosis (A1).

\section{TREATMENT OF CHRONIC HEPATITIS C OR COMPENSATED CIRRHOSIS PATIENTS WITH HCV GENOTYPE 4 INFECTION}

The prevalence of genotype 4 chronic hepatitis $C$ is estimated to be up to $20 \%$ of all hepatitis C patients, mainly in sub-Saharan Africa and Middle Eastern countries. The prevalence of genotype 4 chronic hepatitis $\mathrm{C}$ in Korea is extremely low, so no reports have been made about the treatment efficacy of this genotype in Korea.

\section{Ledipasvir/sofosbuvir}

One phase 2 clinical trial using ledipasvir/sofosbuvir for 12 weeks in $21 \mathrm{CHC}$ patients (cirrhosis $\mathrm{n}=7$, treatment experience $\mathrm{n}=8$ ) showed an SVR of 95\% (20 patients), ${ }^{96}$ and another phase 2 trial with the same treatment in $44 \mathrm{CHC}$ patients (cirrhosis $n=10$, treatment experience $n=22$ ) showed an SVR of 93\% (41 patients). ${ }^{97}$ Because the number of treatment-experienced genotype 4 CHC patients available for clinical studies was small, the ledipasvir/sofosbuvir regimen could be recommended with a prolonged treatment duration, such as 24 weeks, or the 12 week treatment could be offered in combination with ribavirin in those patients to improve SVR, although the evidence is not yet robust.

\section{Elbasvir/grazoprevir}

Twelve weeks of treatment with elbasvir/grazoprevir achieved an SVR of 100\% (18/18) in treatment-naïve genotype 4 CHC patients, ${ }^{13}$ but the same treatment showed an SVR of $78 \%(7 / 9)$ in treatment-experienced genotype $4 \mathrm{CHC}$ patients, though combination treatment with ribavirin for 12 weeks showed an SVR of $100 \%(15 / 15)$ in the latter population. ${ }^{49} \mathrm{~A}$ pooled analysis of phase 2 and 3 clinical trials using elbasvir/grazoprevir analyzed 66 treatment-naïve and 37 treatment-experienced genotype $4 \mathrm{CHC}$ patients. Among them, the treatment-naïve patients and patients who relapsed after a previous treatment showed an SVR of $96 \%$ (54/56) and 100\% (2/2), respectively, following 12 weeks of elbasvir/grazoprevir treatment. But patients with on-treatment failure (including failure to suppress and breakthrough) in a previous treatment showed a higher SVR when they were treated with elbasvir/grazoprevir combined with ribavirin for 16 weeks than they did on elbasvir/grazoprevir for 12 weeks (12 weeks: 71\% [5/7], 16 weeks: $60 \%$ [3/5], 12 weeks+ribavirin: 91\% [10/11], 16 weeks+ribavirin: $100 \%[5 / 5]){ }^{98}$ In a phase 3 trial, patients co-in- 
The Korean Association for the Study of the Liver (KASL) 2017 KASL clinical practice guidelines: Management of chronic hepatitis C

fected with HIV and HCV (genotypes 1, 4, and 6) showed an SVR of $96 \%$ (210/218) following 12 weeks of elbasvir/grazoprevir. ${ }^{99}$

\section{Ombitasvir/paritaprevir/ritonavir combined with ribavirin}

In a phase 2 trial, ombitasvir/paritaprevir/ritonavir combined with ribavirin for 12 weeks produced an SVR of 100\% (42/42) and $100 \%$ (49/49), respectively, in treatment-naïve and treatment-experienced genotype $4 \mathrm{CHC}$ patients without cirrhosis. ${ }^{100}$ In a phase 3 trial of 120 genotype 4 CHC compensated cirrhosis patients, ombitasvir/paritaprevir/ritonavir combined with ribavirin for 12 or 16 weeks of treatment showed an SVR of $97 \%(57 / 59)$ and $98 \%(60 / 61)$, respectively. ${ }^{101}$

\section{Daclatasvir and sofosbuvir}

Daclatasvir and sofosbuvir combination treatment for 12 weeks achieved an SVR in all 6 patients co-infected with HIV and genotype $4 \mathrm{HCV}^{29}$ In one retrospective study of 47 genotype $4 \mathrm{CHC}$ patients, daclatasvir and sofosbuvir combination therapy for 12 weeks showed an SVR of 100\% (32/32), without additional benefit from adding ribavirin or prolonging the treatment to 24 weeks. $^{102}$ In another retrospective study of 215 genotype 4 CHC patients, including 179 treatment-experienced patients, daclatasvir and sofosbuvir combination treatment with or without ribavirin for 12 or 24 weeks showed an overall SVR of 91\% (195/215). However, in cirrhotic patients, the SVR following daclatasvir and sofosbuvir combination therapy for 12 weeks without ribavirin was reduced to $84 \%$ (53/63), and prolonging the treatment duration to 24 weeks or adding ribavirin improved the SVR to $93 \%$ $(102 / 110)$ and $88 \%(7 / 8)$, respectively. ${ }^{103}$

\section{Glecaprevir/pibrentasvir}

Twelve weeks of treatment with glecaprevir/pibrentasvir showed a 100\% (genotype 4 22/22) SVR rate in a phase 2 clinical trial of genotype 4-6, DAA-naïve CHC patients without cirrhosis (treatment experience $15 \%){ }^{36}$ One phase 3 trial also showed an SVR of 99\% (genotype 4 75/76, genotype 5 26/26, genotype 6 19/19). ${ }^{104}$ In that phase 3 trial, the one genotype 4 CHC patient who did not achieve SVR prematurely left the study at day 12. In another phase 3 trial of genotype 1-6 CHC patients, including some cirrhotic patients (treatment experience 25\%: interferon based 17\%, sofosbuvir based $8 \%$ ) a 12-week glecaprevir/pibrentasvir treatment showed an SVR of $100 \%$ in 16 genotype 4 CHC patients. ${ }^{40}$ In an integrated analysis of phase 2 and 3 clinical trials using glecaprevir/pibrentasvir in genotype 1-6 CHC patients without cirrhosis, 8 week and 12 week treatments of glecaprevir/pibrentasvir showed an SVR of 93\% (43/46) and 99\% (111/112), respectively, in genotype 4 patients. ${ }^{38}$

\section{Sofosbuvir/velpatasvir}

Twelve weeks of sofosbuvir/velpatasvir treatment showed an SVR of $100 \%$ in a phase 3 clinical trial of 116 genotype 4 CHC patients (cirrhosis 20\%, treatment experience $45 \%$ ). ${ }^{42}$ In another phase 3 trial of HIV/HCV co-infected patients, 12 weeks of sofosbuvir/velpatasvir treatment achieved an SVR of $100 \%$ SVR in 4 genotype 4 patients. ${ }^{105}$

\section{Sofosbuvir/velpatasvir/voxilaprevir}

In a phase 3 clinical trial of DAA-naïve genotype 1-6 CHC patients (cirrhosis 18\%, treatment experience 23\%), 8 and 12 weeks of sofosbuvir/velpatasvir/voxilaprevir treatment showed an SVR of $94 \%$ (59/63) and 98\% (56/57), respectively, in genotype 4 patients. ${ }^{43}$

Table 9. Treatment of genotype 3 chronic hepatitis $C$ patients with or without compensated cirrhosis

\begin{tabular}{|c|c|c|c|c|}
\hline & \multicolumn{2}{|c|}{ Treatment naive } & \multicolumn{2}{|c|}{ PR experienced } \\
\hline & Chronic hepatitis & Compensated cirrhosis & Chronic hepatitis & Compensated cirrhosis \\
\hline Daclatasvir+sofosbuvir & 12 wk & $24 w k+R$ & $12 w k+R$ & $24 w k+R$ \\
\hline Elbasvir/grazoprevir+sofosbuvir & & & & 12 wk \\
\hline Glecaprevir/pibrentasvir & $8 w k$ & $12 w k$ & $16 w k$ & $16 w k$ \\
\hline Sofosbuvir/velpatasvir & $12 w k$ & $12 w k+R$ & $12 w k+R$ & $12 w k+R$ \\
\hline Sofosbuvir/velpatasvir/voxilaprevir & & $8 w k$ & & $8 w k$ \\
\hline$P R$ & 24 wk & $24 w k$ & & \\
\hline
\end{tabular}

$P R$, pegylated interferon alpha+ribavirin $800 \mathrm{mg}$; wk, weeks; $R$, weight-based ribavirin. 


\section{[Recommendations] (Table 10)}

Initial treatment of chronic hepatitis $\mathrm{C}$ or compensated cirrhosis patients with HCV genotype 4 infection

1. Ledipasvir/sofosbuvir should be administered for 12 weeks (A1).

2. Elbasvir/grazoprevir should be administered for 12 weeks (A1).

3. Ombitasvir/paritaprevir/ritonavir and ribavirin should be administered for 12 weeks (A1).

4. Daclatasvir and sofosbuvir could be administered for 12 weeks to patients without liver cirrhosis (B1).

Daclatasvir, sofosbuvir and ribavirin could be administered for 12 weeks to patients with liver cirrhosis, or they could receive daclatasvir and sofosbuvir for 24 weeks (B1).

5. Glecaprevir/pibrentasvir should be administered for 8 weeks to patients without liver cirrhosis (A1).

Glecaprevir/pibrentasvir should be administered for 12 weeks to patients with liver cirrhosis (A1).

6. Sofosbuvir/velpatasvir should be administered for 12 weeks (A1).

Retreatment of treatment-experienced chronic hepatitis $C$ or compensated cirrhosis patients with HCV genotype 4 infection

1. Ombitasvir/paritaprevir/ritonavir and ribavirin should be administered for 12 weeks (A1).

2. Ledipasvir/sofosbuvir and ribavirin could be administered for 12 weeks or ledipasvir/sofosbuvir could be administered for 24 weeks (B1).

3. Elbasvir/grazoprevir could be administered for 12 weeks to patients with virologic relapse, and elbasvir/grazoprevir and ribavirin could be administered for 16 weeks to patients with on-treatment virologic failure (failure to suppress and breakthrough) (B1).

4. Daclatasvir and sofosbuvir could be administered for 12 weeks to patients without liver cirrhosis (B1).

Daclatasvir, sofosbuvir and ribavirin for 12 weeks or daclatasvir and sofosbuvir for 24 weeks could be administered to patients with liver cirrhosis (B1).

5. Glecaprevir/pibrentasvir should be administered for 8 weeks to patients without liver cirrhosis (A1).

Glecaprevir/pibrentasvir should be administered for 12 weeks to patients with liver cirrhosis (A1).

6. Sofosbuvir/velpatasvir should be administered for 12 weeks (A1)

\section{TREATMENT OF CHRONIC HEPATITIS C OR COMPENSATED CIRRHOSIS PATIENTS WITH HCV GENOTYPE 5 OR 6 INFECTION}

Genotype 5 chronic hepatitis C patients are mainly distributed in Southern Africa, and there has been no report of such a patient in Korea yet. ${ }^{106}$ Genotype $6 \mathrm{CHC}$ patients are found mainly in Southeastern Asia; southern part of China, Hong Kong, and Macao. The prevalence in Korea is only around $1 \%{ }^{106}$ Because of the paucity of genotype 5 and 6 CHC patients in Korea, clinical studies of these patients are few.

\section{Ledipasvir/sofosbuvir}

A phase 2 clinical trial using 12 weeks of ledipasvir/sofosbuvir in 41 genotype 5 CHC patients (cirrhosis $n=9$, treatment experience $n=20)$ showed an SVR of $95 \%(39 / 41),{ }^{104}$ and another study with the same regimen in 25 genotype 6 CHC patients (cirrhosis $n=2$, treatment experience $n=2$ ) showed an SVR of $96 \%$ $(24 / 25){ }^{81}$ In a community-based retrospective study, 12 weeks of ledipasvir/sofosbuvir treatment achieved an SVR of 95\% (62/65) in Asian HCV genotype 6 patients. ${ }^{107}$

\section{Elbasvir/grazoprevir}

Twelve weeks of elbasvir/grazoprevir treatment showed an SVR of $80 \%(8 / 10)$ in treatment naïve genotype 6 CHC patients, ${ }^{13}$ and 16 weeks of elbasvir/grazoprevir with and without ribavirin achieved an SVR of $100 \%(2 / 2)$ and $75 \%(3 / 4)$, respectively, in treatment-experienced genotype $6 \mathrm{CHC}$ patients. ${ }^{49}$

Table 10. Treatment of chronic hepatitis $C$ or compensated cirrhosis patients with hepatitis $C$ virus genotype 4 infection

\begin{tabular}{lcccccr} 
& \multicolumn{2}{c}{ Treatment naive } & & \multicolumn{2}{c}{ PR experienced } \\
& & Chronic hepatitis & Compensated cirrhosis & & Chronic hepatitis & Compensated cirrhosis \\
\hline Ledipasvir/sofosbuvir & $12 w k$ & $12 w k$ & $12 w k+R / 24 w k$ & $12 w k+R / 24 w k$ \\
Elbasvir/grazoprevir & $12 w k$ & $12 w k$ & & $12 w k$ (relapse), $16 w k+R$ & $12 w k$ (relapse), $16 w k+R$ \\
(on-treatment failure) & (on-treatment failure)
\end{tabular}

PR, pegylated interferon+ribavirin; wk, weeks; $R$, weight-based ribavirin; on-treatment failure, including failure to suppress and breakthrough. 
The Korean Association for the Study of the Liver (KASL) 2017 KASL clinical practice guidelines: Management of chronic hepatitis C

\section{Daclatasvir and sofosbuvir}

There has been no report using daclatasvir and sofosbuvir combination treatment in genotype 5 or 6 CHC patients. In a retrospective study, daclatasvir and sofosbuvir combination therapy for 12 or 24 weeks both showed an SVR of $100 \%$ (25/25, 5/5, respectively). ${ }^{103}$

Because the number of treatment-experienced genotype 5 and 6 CHC patients is small in available clinical studies, a ledipasvir/ sofosbuvir regimen or daclatasvir and sofosbuvir combination treatment could be recommended, with a treatment duration of 24 weeks or 12 weeks in combination with ribavirin in these patients, although the evidence is not yet robust.

\section{Glecaprevir/pibrentasvir}

Twelve weeks of glecaprevir/pibrentasvir treatment showed an SVR of 100\% (genotype 4 1/1, genotype 6 11/11) in a phase 2 clinical trial of genotype 4-6, DAA naïve CHC patients without cirrhosis (treatment experience 15\%). ${ }^{36}$ That same regimen also showed an SVR of 99\% (genotype 4 75/76, genotype 5 26/26, genotype 6 19/19) in a phase 3 trial. $^{104}$ In another phase 3 trial of genotype 1-6 CHC patients, including some cirrhotic patients (treatment experience 25\%: interferon based 17\%, sofosbuvir based $8 \%$ ), 12 weeks of glecaprevir/pibrentasvir treatment showed an SVR of $100 \%$ in 2 genotype 5 patients and 7 genotype 6 patients. ${ }^{40}$ In an integrated analysis of phase 2 and 3 clinical trials using glecaprevir/pibrentasvir in genotype 1-6 CHC patients without cirrhosis, 8 weeks and 12 weeks of glecaprevir/ pibrentasvir treatment showed an SVR of 100\% (2/2) and 100\% (28/28), respectively, in genotype 5 patients and $90 \%(9 / 10)$ and $100 \%(31 / 31)$, respectively, in genotype 6 patients. $^{38}$

\section{Sofosbuvir/velpatasvir}

Twelve weeks of sofosbuvir/velpatasvir treatment showed an SVR of $97 \%$ (34/35) in 35 genotype 5 CHC patients (cirrhosis $14 \%$, treatment experience 31\%) and 100\% (41/41) in 41 genotype 6 CHC patients (cirrhosis 15\%, treatment experienced $7 \%$ ) in a phase 3 clinical trial. ${ }^{42}$

\section{Sofosbuvir/velpatasvir/voxilaprevir}

In a phase 3 clinical trial of DAA naïve genotype 1-6 CHC patients (cirrhosis 18\%, treatment experience $23 \%$ ), 8 weeks of so- fosbuvir/velpatasvir/voxilaprevir treatment showed an SVR of 94\% $(17 / 18)$ in genotype 5 patients. Eight weeks of sofosbuvir/velpatasvir/voxilaprevir and 12 weeks of sofosbuvir/velpatasvir treatment showed an SVR of $100 \%$ and 100\% (30/30, 9/9, respectively) in genotype 6 patients. ${ }^{43}$

\section{Peginterferon alpha and ribavirin}

The SVR in chronic HCV genotype 5 or 6 patients treated with a combination of peginterferon alpha and ribavirin was 70-86\%, which is comparable with that of HCV genotype 3 and higher than that of HCV genotype $1 .^{108-110}$ If DAAs are unavailable, a previous standard therapy, such as a combination of peginterferon alpha and ribavirin for 24 weeks, remains acceptable.

\section{[Recommendations] (Table 11)}

Initial treatment of chronic hepatitis $\mathrm{C}$ or compensated cirrhosis patients with HCV genotype 5 or 6 infection

1. Ledipasvir/sofosbuvir should be administered for 12 weeks (A1).

2. Daclatasvir and sofosbuvir could be administered for 12 weeks (B1).

3. Glecaprevir/pibrentasvir should be administered for 8 weeks to patients without liver cirrhosis (A1).

Glecaprevir/pibrentasvir should be administered for 12 weeks to patients with liver cirrhosis (A1).

4. Sofosbuvir/velpatasvir should be administered for 12 weeks (A1).

5. Peginterferon alpha and weight-based ribavirin could be administered for 24 weeks (A2).

Retreatment of treatment-experienced chronic hepatitis $C$ or compensated cirrhosis patients with HCV genotype 5 or 6 infection

1. Ledipasvir/sofosbuvir and ribavirin could be administered for 12 weeks or ledipasvir/sofosbuvir could be administered for 24 weeks (B1).

2. Daclatasvir, sofosbuvir, and ribavirin could be administered for 12 weeks, or daclatasvir and sofosbuvir could be administered for 24 weeks (B1).

3. Glecaprevir/pibrentasvir should be administered for 8 weeks to patients without liver cirrhosis (A1)

Glecaprevir/pibrentasvir should be administered for 12 weeks to patients with liver cirrhosis (A1).

4. Sofosbuvir/velpatasvir should be administered for 12 weeks (A1).

\section{TREATMENT OF PATIENTS WITH DECOMPEN-} SATED CIRRHOSIS

Liver transplantation is the only treatment option for patients 
with end-stage liver disease, but HCV infection frequently recurs due to graft infection after transplantation, which is a major cause of morbidity and mortality in patients undergoing liver transplantation. Controversy remains about whether to treat HCV infection before or after transplantation in patients with decompensated liver disease. It is anticipated that patients successfully achieving an SVR to anti-HCV therapy prior to transplantation would have improvements in liver function, allowing liver transplantation to be avoided. However, response to antiviral therapy before transplantation is reportedly worse than that from treating early HCV recurrence after transplantation, and thus, unsuccessful pre-transplant antiviral therapy can lead to missed opportunities to cure end-stage liver disease with transplantation. ${ }^{111}$ Therefore, the decision to treat HCV in patients with impaired hepatic function should be individualized, with consideration of the indications for transplantation, the availability of liver grafts, and the feasibility of liver transplantation. In general, a combination therapy of sofosbuvir and NS5A inhibitors such as ledipasvir, daclatasvir, or velpatasvir is recommended for the treatment of patients with decompensated cirrhosis (Table 12).

In a multicenter controlled study of 108 patients with HCV genotypes 1 and 4 with decompensated cirrhosis (CTP class B or C, CTP scores $\leq 12$ ), participants were randomly assigned to receive ledipasvir/sofosbuvir and ribavirin (initial dose of $600 \mathrm{mg}$, increased as tolerated) for 12 or 24 weeks. SVR was achieved in $87 \%$ and $89 \%$ of patients given the 12 - and 24 -week treatment courses, respectively. Baseline CTP and Model for End-Stage Liver Disease (MELD) scores improved in more than $50 \%$ of the patients, but some patients experienced worsening of hepatic function. During the treatment, five (5\%) patients died of variceal bleeding. Grade 3 or 4 adverse events occurred in 15\% and 34\% of patients in the 12- and 24-week arms, respectively. ${ }^{112}$

In a phase III study, 60 patients with decompensated cirrhosis (mostly CTP class B and C, HCV genotype 1/3/2, 4, 6=45/6/9) received daclatasvir daily in combination with sofosbuvir and a low initial dose of ribavirin (600 mg) for 12 weeks. ${ }^{33}$ The overall SVR was $83 \%$. SVR was observed in $76 \%$ and $100 \%$ of patients with HCV genotypes $1 \mathrm{a}$ and $1 \mathrm{~b}$, respectively. In patients with HCV genotype 1 infection, the SVR rates were 92\% and 50\% among patients with CTP class B and C, respectively. Among subjects with HCV genotype 3 and 2/4/6, the SVR rates were $83 \%$ and $89 \%$, respectively.

A recent multicenter, open-label trial involving 267 previously treated and untreated patients with CTP class B (genotype 1a, 159; genotype 1b, 48; genotype 2, 12; genotype 3, 39; genotype 4,8 ; and genotype 6,1 ) evaluated the efficacy and safety of sofosbuvir/velpatasvir with or without ribavirin. Subjects were randomly assigned to receive sofosbuvir/velpatasvir once daily for 12 weeks, sofosbuvir/velpatasvir plus ribavirin for 12 weeks, or sofosbuvir/velpatasvir for 24 weeks. The SVR was $88 \%, 94 \%$, and $93 \%$, respectively, in patients with genotype 1a, and $89 \%, 100 \%$, and $88 \%$, respectively, in patients with genotype $1 \mathrm{~b}$. The corre-

Table 11. Treatment of chronic hepatitis C or compensated cirrhosis patients with hepatitis C virus genotype 5 or 6 infection

\begin{tabular}{|c|c|c|c|c|}
\hline & \multicolumn{2}{|c|}{ Treatment naive } & \multicolumn{2}{|c|}{ PR experienced } \\
\hline & Chronic hepatitis & Compensated cirrhosis & Chronic hepatitis & Compensated cirrhosis \\
\hline Ledipasvir/sofosbuvir & $12 w k$ & 12 wk & $12 w k+R / 24 w k$ & $12 w k+R / 24 w k$ \\
\hline Sofosbuvir+daclatasvir & $12 w k$ & $12 w k$ & $12 w k+R / 24 w k$ & $12 w k+R / 24 w k$ \\
\hline Glecaprevir/pibrentasvir & $8 w k$ & 12 wk & $8 w k$ & $12 w k$ \\
\hline Sofosbuvir/velpatasvir & $12 w k$ & 12 wk & $12 w k$ & $12 w k$ \\
\hline$P R$ & $24 w k$ & $24 w k$ & & \\
\hline
\end{tabular}

$\mathrm{PR}$, pegylated interferon+ribavirin; wk, weeks; $R$, weight-based ribavirin.

Table 12. Treatment of decompensated cirrhosis

\begin{tabular}{lcc} 
& Genotype 1, 4, 5, 6 & Genotype 2, 3 \\
\hline Ledipasvir/sofosbuvir & $12 w k+R^{*} / 24 w k$ & $12 w k+R^{*} / 24 w k$ \\
Daclatasvir+sofosbuvir & $12 w k+R^{*} / 24 w k$ & $12 w k+R / 24 w k$ \\
\hline Sofosbuvir/velpatasvir & $12 w k+R / 24 w k$ & $w$ \\
\hline
\end{tabular}

wk, weeks; $R$, weight-based ribavirin.

*Ribavirin started from $600 \mathrm{mg} / \mathrm{d}$. 
The Korean Association for the Study of the Liver (KASL) 2017 KASL clinical practice guidelines: Management of chronic hepatitis C

sponding rates were $100 \%, 100 \%$, and $75 \%$, for patients with genotype 2 and the rates were $50 \%, 85 \%$, and $50 \%$, for patients with genotype 3 , respectively. Among patients with HCV genotype 4, all (100\%) achieved an SVR to the sofosbuvir/velpatasvirbased regimens. ${ }^{113}$

In a retrospective study involving 409 patients with decompensated cirrhosis who were treated with sofosbuvir, ledipasvir, or daclatasvir, with or without ribavirin, the SVR was $85 \%$ (11/13) in patients with genotype 1 with ledipasvir/sofosbuvir for 12 weeks, $91 \%(136 / 149)$ in those with ribavirin added to ledipasvir/sofosbuvir for 12 weeks, 50\% (2/4) in those with daclatasvir and sofosbuvir for 12 weeks, and 88\% (30/34) in those with ribavirin added to daclatasvir and sofosbuvir for 12 weeks. Among patients with HCV genotype 3, an SVR was achieved in 60\% (3/5) of patients who received daclatasvir and sofosbuvir for 12 weeks and in $71 \%(75 / 105)$ of patients with ribavirin added to daclatasvir and sofosbuvir for 12 weeks. ${ }^{114}$ Improvement in the MELD score was more frequently observed in patients with DAA treatment than in those without. The benefits of DAA treatment were less likely to be found among patients who had hypoalbuminemia $(<3.5 \mathrm{~g} / \mathrm{dL})$ or hyponatremia ( $<135 \mathrm{mmol} / \mathrm{L})$ at baseline, as well as among elderly patients ( $>65$ years).

Data on the efficacy of 24 weeks of daclatasvir and sofosbuvir with or without ribavirin for decompensated liver disease was reported from 165 European patients (CTP class B 143, CTP class C 22; genotype 1,73\%; genotype 3, 21\%; genotype 4,4\%). The SVR rate was $86 \%(115 / 134)$ in patients with CTP class B and $76 \%(16 / 21)$ in patients with CTP class C. Among patients with genotype 3, response to a 24-week combination therapy of daclatasvir, sofosbuvir, and ribavirin was $87 \%(13 / 15)$ in the CTP class B group and $100 \%(2 / 2)$ in the CTP class C group. The overall response rates varied according to baseline liver function, with significantly low SVR rates in patients with CTP class C or MELD scores of $\geq 16^{86}$

Taken together, those results reveal that DAA therapy can improve liver function in $50-80 \%$ of patients with advanced liver disease, even with decompensated cirrhosis. However, a subset of patients with severe decompensation might not improve or might even progress after DAA therapy, suggesting that there could be a point of no return at which anti-HCV therapy cannot reverse liver dysfunction. In a study involving 92 Australian cirrhotic patients with MELD scores of $\geq 15$ who received a combination of daclatasvir and sofosbuvir without ribavirin for 24 weeks, improvement in liver function (a decrease in MELD scores by $\geq 2$ ) after DAA therapy was observed in $50 \%$ of the whole patient group and was lim- ited to patients with baseline MELD scores $\leq 20$. Therefore, an improvement in liver function following DAA therapy is unlikely in patients with a baseline MELD score of $\geq 20$. $^{115}$

Among DAAs, PIs (asunaprevir, paritaprevir, grazoprevir) and dasabuvir are contraindicated in patients with decompensated cirrhosis (CTP class B and C) and in those with a past history of decompensated complications due to drug toxicity from increased drug concentrations. The recently developed DAA drugs, glecaprevir and voxilaprevir, are also contraindicated in patients with CTP class B or $C$ because of significant changes in plasma drug concentrations. Among patients with compensated cirrhosis, hepatic failure and mortality were reported during the first 1-4 weeks of paritaprevir, ombitasvir, and dasabuvir therapy. ${ }^{116}$

There is very little information on DAA therapy for decompensated patients with CTP class C. Efficacy and safety data in patients with more advanced liver disease (CTP scores $\geq 13$ ) are limited and require verification, due to a lack of clinical study. Patients with advanced liver disease should be regularly monitored for consideration of liver transplantation because the risk of liver cancer or progressive liver disease is not eliminated, even in patients achieving an SVR to anti-HCV therapy. ${ }^{117}$

With the advent of interferon-free, all-oral DAA regimens, successful treatment of HCV infection can be considered in patients with decompensated cirrhosis. However, anti-HCV treatment for this particular group is complicated and should be carefully balanced against its potential disadvantages. In fact, overall response rates to pre-transplant DAA therapy are still lower than those from treating early HCV recurrence after transplantation. ${ }^{111}$ Therefore, the decision to treat HCV infection in patients with decompensated cirrhosis should be individualized under consideration of the accessibility of transplantation and the waiting time for liver transplantation.

\section{[Recommendations] (Table 12)}

General recommendations

1. Patients with decompensated cirrhosis (CTP class B or C) and HCV viremia should be referred to a medical specialist or liver transplant center ( $\mathrm{C} 1)$

2. Patients can be treated for HCV infection if the waiting time for liver transplantation is more than 6 months or if liver transplantation is infeasible (B1)

3. Particular care should be taken to monitor drug-related adverse events or toxicity when treating patients on the waiting list for transplantation or those with severe decompensation (B1).

4. Protease inhibitors should not be used in patients with decompensated cirrhosis due to drug-related adverse effects (A1). 


\section{DAA therapy}

1. Treatment of patients infected with HCV genotypes $1,4,5$, or 6 who have decompensated cirrhosis

(1) Ledipasvir/sofosbuvir with a low initial dose of ribavirin $(600 \mathrm{mg}$, increased as tolerated) should be administered for 12 weeks (A1). Ledipasvir/sofosbuvir could be administered for 24 weeks to patients who are ribavirin intolerant or ineligible (B1).

(2) Daclatasvir and sofosbuvir with a low initial dose of ribavirin (600 $\mathrm{mg}$, increased as tolerated) should be administered for 12 weeks (A1).

Daclatasvir and sofosbuvir could be administered for 24 weeks to patients who are ribavirin intolerant or ineligible (B1).

(3) Sofosbuvir/velpatasvir with weight-based ribavirin (1,200 mg in patients $\geq 75 \mathrm{~kg}, 1,000 \mathrm{mg}$ in patients $<75 \mathrm{~kg}$ ) should be administered for 12 weeks (A1).

Sofosbuvir/velpatasvir could be administered for 24 weeks to patients who are ribavirin intolerant or ineligible (B1).

2. Treatment of patients infected with HCV genotype 2 or 3 who have decompensated cirrhosis

(1) Daclatasvir and sofosbuvir with a low initial dose of ribavirin (600 $\mathrm{mg}$, increased as tolerated) should be administered for 12 weeks (A1).

Daclatasvir and sofosbuvir could be administered for 24 weeks to patients who are ribavirin intolerant or ineligible (B1).

(2) Sofosbuvir/velpatasvir with weight-based ribavirin (1,200 mg in patients $\geq 75 \mathrm{~kg}, 1,000 \mathrm{mg}$ in patients $<75 \mathrm{~kg}$ ) should be administered for 12 weeks (A1)

Sofosbuvir/velpatasvir could be administered for 24 weeks to patients who are ribavirin intolerant or ineligible (B1).

\section{LIVER TRANSPLANTATION AND OTHER OR- GAN TRANSPLANTS}

\section{Treatment before liver transplantation}

HCV reinfection occurs within several hours after transplantation in most patients with detectable HCV RNA at the time of transplantation. ${ }^{118}$ Thus, patients with HCV infection at the time of $L T$ have a higher graft failure rate (hazard ratio [HR], 1.30; 95\% confidence interval $[\mathrm{CI}], 1.21-1.39)$ and mortality rate $(\mathrm{HR}, 1.23$; $95 \% \mathrm{Cl}, 1.12-1.35)$ than patients without HCV infection. ${ }^{119} \mathrm{HCV}$ related liver diseases rapidly deteriorate following liver transplantation, and around one-third of patients progress to cirrhosis within 5 years after transplantation. ${ }^{120}$ Therefore, successful elimination of HCV before or after transplantation is critical to improving the prognosis of the graft and patient.

Treatment of HCV infection in patients awaiting transplantation has two goals: i) preventing liver graft infection after transplanta- tion and ii) improving liver function before transplantation in patients with decompensated cirrhosis. The improvement of liver function could lead to delisting of some of patients awaiting transplantation. ${ }^{111}$ However, the duration of DAA therapy might be insufficient in a patient on the waiting list because the time to liver transplantation is unpredictable.

In a phase 2, open-label study, 61 patients infected with HCV genotype $1,2,3$, or 4 were treated with sofosbuvir and ribavirin for up to 48 weeks prior to transplantation; 46 of those patients received transplants. Among the 43 patients with an HCV RNA level $<25 \mathrm{IU} / \mathrm{mL}$ at the time of transplantation, $30(70 \%)$ had an SVR post-transplantation. The duration of undetectable HCV RNA before transplantation was the best predictor of a post-treatment response. HCV recurrence occurred in 64\% (9/14) versus 4\% $(1 / 26)$ in patients with undetectable HCV RNA for less than 30 continuous days versus more than 30 days before transplantation, respectively. ${ }^{121}$

According to consensus statements from the European Liver and Intestine Transplant Association on the use of DAAs in the setting of liver transplantation, patients with a baseline MELD $\leq 20$ have a $12-35 \%$ chance of being delisted after DAA therapy because of clinical improvement, and they should thus be treated while listed. In contrast, patients with high baseline MELD scores (>25) are not recommended for pre-transplant DAA treatment because of an unknown probability of improvement post-treatment and potential DAA toxicity. ${ }^{122}$ In fact, an analysis of pooled data from studies of various DAA therapies for recurrent hepatitis $C$ in transplant recipients showed an overall SVR greater than $85 \%$. $^{111}$ The SVR is reported to be even greater post-transplantation, reaching $91-100 \%$, when DAA therapy is initiated early in the relapse after transplantation. Therefore, it is advantageous to treat such patients post-transplantation rather than pre-transplantation, when their liver dysfunction is severe. ${ }^{33,112}$ For patients with MELD scores between 21 and 25, a case-by-case multidisciplinary decision on which patients to treat is advised..$^{122}$

\section{Treatment following liver transplantation}

All patients with post-transplant recurrence of HCV infection should be prioritized for antiviral therapy. In particular, antiviral treatment should be started as soon as possible when fibrosing cholestatic hepatitis, advanced fibrosis, or portal hypertension is noted because those conditions predict a rapid progression of liver disease and graft failure.

In a multicenter study involving transplant recipients $(n=229)$ 
The Korean Association for the Study of the Liver (KASL) 2017 KASL clinical practice guidelines: Management of chronic hepatitis C

infected with HCV genotype 1 or 4, study participants were randomly assigned to receive ledipasvir/sofosbuvir and weight-based ribavirin $(1,000 \mathrm{mg}[<75 \mathrm{~kg}]$ to $1,200 \mathrm{mg}[>75 \mathrm{~kg}])$ for either 12 or 24 weeks. In patients with Metavir fibrosis stages F0 to F3, an SVR was achieved in $96 \%$ and $98 \%$ of patients in the 12 - and 24 week arms, respectively. In patients with compensated cirrhosis, an SVR was achieved in $96 \%$ of patients in both the 12- and 24week arms. Efficacy was lower in patients with CTP class B cirrhosis (SVR $85 \%$ vs. $88 \%$ in the 12- and 24-week arms) or CTP class C cirrhosis (60\% vs. $75 \%$ in the 12 - and 24 -week arms). ${ }^{112}$ Similar results were recorded in another study, which also assessed a combination therapy of ledipasvir/sofosbuvir and ribavirin for 12 or 24 weeks in transplant recipients with recurrent hepatitis C. Genotype 1 patients without cirrhosis had an SVR of 93\% (42/45) with 12 weeks of therapy and 100\% (44/44) with 24 weeks of therapy. When analyzed according to baseline CTP class, the SVR following 12 and 24 weeks of therapy was 100\% (30/30) and 96\% (27/28) in patients with CTP class A, 95\% (19/20) and 100\% (20/20) in CTP class B, and 50\% (1/2) and 80\% (4/5) in CTP class $C$, respectively. ${ }^{123}$

In an open-label study, daclatasvir was administered in combination with daily sofosbuvir and ribavirin (initial dose, $600 \mathrm{mg}$ ) for 12 weeks to patients with recurrent HCV infection post-transplant ( $n=53$, HCV genotype $1 / 3 / 2,4,6=41 / 11 / 1)$. Overall, $94 \%$ of the patients achieved an SVR. The SVR was 95\% (39/41), 91\% (10/11), and 100\% (1/1) in patients infected with genotypes 1, 3, and $2 / 4 / 6$, respectively. ${ }^{33}$ The median dose of ribavirin given to the patients was $480 \mathrm{mg} / \mathrm{d}$, with no drug-related adverse events.
Recently, the safety and efficacy of glecaprevir/pibrentasvir was investigated in 100 transplant recipients (genotype 1, 57\%, genotype 2, 13\%, genotype 3, 24\%, genotype 4-6,6\%) who developed recurrent HCV infection (F0-F1 [80\%], F2 [6\%], and F3 [14\%]) after liver transplantation $(n=80)$ or renal transplantation $(n=20)$. The overall SVR was $98 \%$ after 12 weeks of therapy with glecaprevir/pibrentasvir. ${ }^{124}$ The efficacy of another DAA, such as sofosbuvir/velpatasvir, is currently being studied in patients with recurrent hepatitis C post-transplant.

The use of ombitasvir/paritaprevir/ritonavir plus dasabuvir and weight-based ribavirin for 24 weeks was assessed in patients with HCV infection who had no or mild fibrosis post-transplantation. The study involving 34 transplant recipients with Metavir fibrosis stage F0-F2 and an HCV genotype 1 infection (genotype 1a 29) reported an SVR of $97 \%$ (33/34). The most common adverse events were fatigue, headache, and cough. Two patients (6\%) had serious adverse events; no patient died from treatment-related adverse events. Most patients received $600-800 \mathrm{mg}$ of ribavirin at study initiation and at the completion of treatment. Overall, 19 patients (56\%) had a modification in the ribavirin dose during treatment, and five patients (15\%) required erythropoietin. Dosages of calcineurin inhibitors were modified because of drugdrug interactions with ritonavir and paritaprevir. ${ }^{125}$ Drug interactions can also occur with elbasvir/grazoprevir, which is contraindicated in post-transplant recipients receiving cyclosporine. In general, $\mathrm{PI}$-containing regimens are not recommended for the treatment of relapsed hepatitis $C$ after liver transplantation. ${ }^{126}$ Table 13 summarizes DAA interactions with calcineurin inhibitors

Table 13. Direct-acting antivirals interactions with calcineurin inhibitors

\begin{tabular}{|c|c|c|}
\hline & Cyclosporine & Tacrolimus \\
\hline Daclatasvir & No clinically significant DDI observed & No clinically significant DDI observed \\
\hline Sofosbuvir & $\begin{array}{l}\text { An increase ( } 4.5 \text {-fold) in SOF AUC, but no a priori dose } \\
\text { adjustment required }\end{array}$ & No clinically significant DDI observed \\
\hline Ledipasvir/sofosbuvir & No clinically significant DDI observed & No clinically significant DDI observed \\
\hline $\begin{array}{l}\text { Ombitasvir/paritaprevir/ritonavir } \\
\text { plus dasabuvir }\end{array}$ & $\begin{array}{l}\text { An increase (5.8-fold) in CSA AUC; suggest using } 1 / 5 \text { of } \\
\text { CSA dose during OPr-D therapy with monitoring of } \\
\text { CSA levels and titration of CSA dose }\end{array}$ & $\begin{array}{l}\text { An increase (57-fold) in TAC AUC; suggest using } \\
\text { TAC } 0.5 \text { mg every } 7 \text { days during OPr-D therapy } \\
\text { with monitoring of TAC levels and titration of TAC } \\
\text { dose }\end{array}$ \\
\hline Elbasvir/grazoprevir & $\begin{array}{l}\text { 15-fold increase in GZR AUC and 2-fold increase in EBR } \\
\text { AUC; not recommended }\end{array}$ & $\begin{array}{l}\text { An increase (43\%) in TAC, but no a priori dose } \\
\text { adjustment required }\end{array}$ \\
\hline Sofosbuvir/velpatasvir & No clinically significant DDI observed & No clinically significant DDI observed \\
\hline Glecaprevir/pibrentasvir & $\begin{array}{l}\text { An increase in G/P AUC; not recommended in patients } \\
\text { requiring stable CSA doses }>100 \text { mg per day }\end{array}$ & $\begin{array}{l}\text { Potential DDI requiring a dose adjustment } \\
\text { expected }\end{array}$ \\
\hline
\end{tabular}

DDI, drug-drug interaction; SOF, sofosbuvir; AUC, area under the plasma concentration curve; CSA, cyclosporine; TAC, tacrolimus; OPr-D, ombitasvir/ paritaprevir/ritonavir plus dasabuvir; GZR, grazoprevir; EBR, elbasvir; G/P, glecaprevir/pibrentasvir. 
in the treatment of recurrent hepatitis C post-transplantation.

\section{Treatment following other organ transplants}

Renal transplant patients with HCV infection display rapidly progressing hepatic fibrosis and high mortality related to hepatic failure; therefore, antiviral treatment prior to renal transplantation has been recommended in the past. ${ }^{127}$ However, with the introduction of DAAs, successful elimination of HCV after renal transplantation can be achieved. It remains to be determined whether patients with chronic hepatitis $C$ should optimally proceed to renal transplantation with the expectation that their hepatitis $\mathrm{C}$ can be cured post-transplant to improve the outcome. Combination therapy with pegylated interferon a plus ribavirin causes a low SVR of around $18 \%$ and graft rejection in more than $30 \%$ of patients, leading to graft failure and death. Thus, DAA therapy is preferred over interferon-containing therapy in renal transplant patients. ${ }^{128,129}$

In a pooled analysis of 10 studies of 333 patients with renal transplantation (genotype 1, 88\%; treatment naïve, 63\%; liver cirrhosis, 25\%) receiving 12-24 weeks of DAA therapy, sofosbuvir-based regimens were the most commonly used. The overall SVR in post-renal transplant patients treated with DAAs was $94.2 \%$, with an SVR of $67 \%$ (10/15) for the combination of sofosbuvir and ribavirin, $75 \%$ (3/4) for the combination of sofosbuvir and daclatasvir, and 98\% (158/161) for the combination of ledipasvir and sofosbuvir with or without ribavirin. DAA therapy for renal transplant patients resulted in relatively good safety profiles, with drug discontinuation reported in only $2 \%$ of cases and stable kidney and liver function during therapy. ${ }^{129}$ Sofosbuvir-based combination therapies are highly recommended for recurrent hepatitis $\mathrm{C}$ after renal transplantation because of their excellent tolerability and safety. In a recent study of 100 patients undergoing liver and renal transplantation, glecaprevir/pibrentasvir was given for 12 weeks and achieved an SVR of $98 \%$, with no treatment-related adverse events. ${ }^{124}$

Of the currently approved DAAs, sofosbuvir, daclatasvir, and ledipasvir are generally recommended in transplant patients because they do not have drug interactions with the immunosuppressive agents used after transplantation and do not require dosage reduction (Table 14). ${ }^{129}$ No data on anti-HCV therapy before or after a transplant of the heart, lung, pancreas, small intestine, or cornea are available. When anti-HCV therapy is needed, DAA therapy is preferred over interferon-containing therapy.

\section{[Recommendations] (Table 14)}

\section{General recommendations}

1. Antiviral therapy can prevent graft infection in patients awaiting liver transplantation and should follow the recommendations according to liver function and HCV genotype (A1).

2. Patients with decompensated cirrhosis and a MELD score $\leq 20-25$ can be treated as soon as possible prior to liver transplantation. Patients with decompensated cirrhosis awaiting liver transplantation with a MELD score > 20-25 could receive the transplant first without antiviral therapy and could be treated for HCV infection after liver transplantation (B1).

3. All patients who develop recurrent HCV infection after liver transplantation should be prioritized for antiviral therapy (A1).

4. Antiviral treatment should be started as early as possible after liver transplantation (A1) because the development of fibrosing cholestatic fibrosis, advanced fibrosis, or portal hypertension after transplantation predicts a rapid progression of liver disease and graft failure (A1).

\section{DAA therapy}

1. Treatment of patients without cirrhosis or with compensated cirrhosis who develop recurrent HCV infection after liver transplantation: HCV genotype 1, 4, 5, or 6

(1) Ledipasvir/sofosbuvir with weight-based ribavirin (1,200 mg in patients $\geq 75 \mathrm{~kg}, 1,000 \mathrm{mg}$ in patients $<75 \mathrm{~kg}$ ) should be administered for 12 weeks (A1). Ledipasvir/sofosbuvir could be administered for 24 weeks to patients who are ribavirin intolerant or ineligible (B1).

(2) Daclatasvir and sofosbuvir with a low initial dose of ribavirin (600 $\mathrm{mg}$, increased as tolerated) could be administered for 12 weeks (B1). Daclatasvir and sofosbuvir could be administered for 24 weeks to patients who are ribavirin intolerant or ineligible (B1).

(3) Glecaprevir/pibrentasvir could be administered for 12 weeks (B1).

(4) Ombitasvir/paritaprevir/ritonavir plus dasabuvir and weightbased ribavirin $(1,200 \mathrm{mg}$ in patients $\geq 75 \mathrm{~kg}, 1,000 \mathrm{mg}$ in patients $<75 \mathrm{~kg}$ ) could be administered for 24 weeks to patients who have early stage fibrosis (Metavir stage F0-F2) (B1).

2. Treatment of patients with decompensated cirrhosis who develop recurrent HCV infection after liver transplantation: HCV genotype $1,4,5$, or 6

(1) Ledipasvir/sofosbuvir with a low initial dose of ribavirin (600 mg, increased as tolerated) should be administered for 12 weeks (A1). Ledipasvir/sofosbuvir could be administered for 24 weeks to patients who are ribavirin intolerant or ineligible (B1).

(2) Daclatasvir and sofosbuvir with a low initial dose of ribavirin (600 mg, increased as tolerated) could be administered for 12 weeks (B1). Daclatasvir and sofosbuvir could be administered for 24 weeks to patients who are ribavirin intolerant or ineligible (B1).

3. Treatment of patients without cirrhosis or with compensated cirrhosis who develop recurrent HCV infection after liver transplantation: HCV genotype 2 
The Korean Association for the Study of the Liver (KASL)

2017 KASL clinical practice guidelines: Management of chronic hepatitis C

(1) Daclatasvir and sofosbuvir with a low initial dose of ribavirin (600 $\mathrm{mg}$, increased as tolerated) could be administered for 12 weeks (C1).

Daclatasvir and sofosbuvir could be administered for 24 weeks to patients who are ribavirin intolerant or ineligible (C2).

(2) Sofosbuvir and weight-based ribavirin (1,200 mg in patients $\geq 75$ $\mathrm{kg}, 1,000 \mathrm{mg}$ in patients $<75 \mathrm{~kg}$ ) could be administered for 24 weeks (C2).

(3) Glecaprevir/pibrentasvir could be administered for 12 weeks (B1).

4. Treatment of patients with decompensated cirrhosis who develop recurrent HCV infection after liver transplantation: HCV genotype 2

(1) Daclatasvir and sofosbuvir with a low initial dose of ribavirin (600 $\mathrm{mg}$, increased as tolerated) could be administered for 12 weeks (C1)

Daclatasvir and sofosbuvir could be administered for 24 weeks to patients who are ribavirin intolerant or ineligible (C2).

(2) Sofosbuvir with a low initial dose of ribavirin $(600 \mathrm{mg}$, increased as tolerated) could be administered for 24 weeks (C2).

5. Treatment of patients without cirrhosis or with compensated cirrhosis who develop recurrent HCV infection after liver transplantation: HCV genotype 3

(1) Daclatasvir and sofosbuvir with a low initial dose of ribavirin (600 $\mathrm{mg}$, increased as tolerated) could be administered for 12 weeks (B1). Daclatasvir and sofosbuvir could be administered for 24 weeks to patients who are ribavirin intolerant or ineligible (C2).

(2) Glecaprevir/pibrentasvir could be administered for 12 weeks (B1).

6. Treatment of patients with decompensated cirrhosis who develop recurrent HCV infection after liver transplantation: HCV genotype 3

(1) Daclatasvir and sofosbuvir with a low initial dose of ribavirin (600 mg, increased as tolerated) could be administered for 12 weeks (B1). Daclatasvir and sofosbuvir could be administered for 24 weeks to patients who are ribavirin intolerant or ineligible (C2).

7. When DAAs are administered to patients after transplantation, drug-drug interactions with immunosuppressants should be considered (A1).

8. When antiviral therapy is considered in non-hepatic solid organ transplant recipients, DAA therapy is preferred over interferoncontaining therapy (A1).

\section{TREATMENT OF SPECIAL POPULATIONS}

Because clinical trials on patients with specific medical conditions have many limitations, antiviral treatment should be individualized in these populations.

\section{Persons who inject drugs}

Injection drug abuse is the main route of HCV transmission, and abusers show a significantly higher HCV infection rate than those without a history of drug abuse. ${ }^{130}$ Among Korean intravenous drug users, 48.4-79.2\% test positive for anti-HCV antibodies. $^{131,132}$

Narcotics are classified as psychotropic agents (ecstasy and methamphetamine), cannabis, and narcotics in the narrow-sense (heroin and cocaine). Among them, the proportion of psychotropic medicines has increased steadily since 2011, accounting for $81.3 \%$ of abuse according to the Annual Narcotics Crime White Paper published by the Supreme Prosecutor's Office (SPO) in 2015. The treatment of persons who inject drugs (PWID) who have chronic HCV infection significantly reduces liver-related complications and transmission to healthy persons. However, active PWID tend to have an increased likelihood of treatment failure and reinfection if they do not receive adequate support for their drug abuse. Multidisciplinary cooperative treatment among medical and psychiatric counseling services and social support results in a significant increase in adherence to treatment.

A meta-analysis of more than 2,800 injection drug users showed an SVR of $44.9 \%$ in HCV genotype 1 patients and $70.0 \%$ in HCV genotype 2 and 3 patients treated with peginterferon-a and ribavirin. ${ }^{133}$ Treatment outcomes from DAAs have not been fully evaluated in injection drug users, who have been widely excluded from clinical trials of DAAs. A few studies included injection drug users who received opioid agonist therapy. Treatment with ombitasvir/paritaprevir/ritonavir and dasabuvir for 12 weeks

Table 14. Treatment after liver transplantation

\begin{tabular}{lcccc}
\hline & Genotype $\mathbf{1}$ & Genotype 2 & Genotype 3 & Genotype 4, 5, 6 \\
\hline Ledipasvir/sofosbuvir & $12 w k+R / 24 w k$ & & $12 w k+R / 24 w k$ \\
\hline Daclatasvir+sofosbuvir & $12 w k+R^{*} / 24 w k$ & $12 w k+R^{*} / 24 w k$ & $12 w k+R^{*} / 24 w k$ & $12 w k+R^{*} / 24 w k$ \\
Glecaprevir/pibrentasvir ${ }^{\dagger}$ & $12 w k$ & $12 w k$ & $12 w k$ & $12 w k$ \\
Sofosbuvir & & $24 w k+R$ & \\
Ombitasvir/paritaprevir/ritonavir+dasabuvir & $24 w k+R(F 0-F 2)$ & & & \\
\hline
\end{tabular}

wk, weeks; R, weight-based ribavirin in chronic hepatitis and compensated cirrhosis, ribavirin started from $600 \mathrm{mg} / \mathrm{d}$ in decompensated cirrhosis.

${ }^{*}$ Ribavirin started from $600 \mathrm{mg} / \mathrm{d}{ }^{\dagger}{ }^{\dagger}$ Not indicated in decompensated cirrhosis. 
resulted in an SVR12 of 97\% (37/38) in genotype 1 HCV-infected, non-cirrhotic, treatment-naive injection drug users who also received opioid agonist therapy (methadone or buprenorphine). ${ }^{134}$ Also, elbasvir/grazoprevir treatment for 12 weeks resulted in an SVR12 of 93\% (144/154) in genotype 1a HCV-infected, treatmentnaïve patients and $93 \%(28 / 30)$ in genotype $1 \mathrm{~b}$ patients. ${ }^{135}$

Drug-drug interaction studies have reported no clinically important interactions between some DAAs (sofosbuvir, daclatasvir, and elbasvir/grazoprevir) and methadone or buprenorphine. ${ }^{111,134,136-138}$ However, ledipasvir/sofosbuvir can increase the serum concentration of buprenorphine through the inhibition of P-glycoprotein by ledipasvir. Co-administration of ombitasvir/paritaprevir/ritonavir and dasabuvir with buprenorphine or naloxone can also increase the concentration of the opioid agonist. In addition to opioid substitution therapy, antidepressants, antipsychotics, and sedatives are frequently used in patients with addiction problems. It was reported that no drug-drug interaction occurred between escitalopram or citalopram and DAAs. However, no pharmacokinetic data on other psychotropic agents are available ${ }^{111,139}$ Because ombitasvir/paritaprevir/ritonavir and dasabuvir treatment, which inhibits CYP3A4, could interact with psychotropic agents, attention should be paid to potential drug interactions when they are used together.

\section{[Recommendations]}

1. PWID should be treated following the guidelines for persons without drug abuse after managing drug-drug interactions (B1).

2. Multidisciplinary cooperative treatment from medical and psychiatric counseling services, social support by specialists in drug abuse, and improvement in the social environment can increase compliance with treatment among intravenous drug users (A1).

\section{Chronic kidney disease}

HCV infection rates in dialysis patients differ from 3\% to $80 \%$ among regions ${ }^{140}$; anti-HCV positivity rates from the late 1990s to the early 2000s in Korea were 5.9-14.7\%. ${ }^{141-143}$ In contrast, the Dialysis Registry, ESRD Registry Committee of the Korean Society of Nephrology reported an anti-HCV antibody positivity rate of $4 \%$ in 2016. ${ }^{144}$

The HCV infection rate is high in patients with chronic kidney disease (CKD). However, anti-HCV screening might not be needed for these patients. Screening should be selectively conducted when HCV-related glomerulonephritis clinically presenting as he- maturia, albuminuria, or cryoglobulinemia is suspected. However, anti-HCV antibody testing should be done in patients undergoing maintenance dialysis for the first time or who were transferred from other dialysis units. In addition, when unexplained abnormal liver-related biochemical tests are found or HCV exposure is suspected, anti-HCV antibodies should be tested, and HCV RNA assays should be performed in patients who are continously negative for anti-HCV antibodies. ${ }^{145}$ The optimal interval for surveillance of HCV infection in anti-HCV negative patients in dialysis units is 6-12 months, taking into consideration the HCV infection rate of the dialysis unit in question.

Patients with CKD show a higher rate of progression to ERSD if they have HCV infection, and it was reported that dialysis patients with HCV infection had a higher mortality rate than those without. ${ }^{146,147}$ Patients scheduled for kidney transplantation should receive an anti-HCV assay and consider HCV treatment because the survival rate after kidney transplantation tends to be low in patients with HCV infection, with a higher possibility of graft rejection and increased occurrence of diabetes and membranous nephritis compared with patients without HCV infection. ${ }^{142}$ Interferon-based antiviral therapy is not recommended after kidney transplantation due to possible graft rejection ${ }^{148-151}$; however, DAA-based antiviral therapy can be safely applied after kidney transplantation.

Indications for HCV treatment in CKD patients should be determined considering liver disease conditions and therapeutic complications. Sofosbuvir, ledipasvir/sofosbuvir, elbasvir/grazoprevir, ombitasvir/paritaprevir/ritonavir with dasabuvir, daclatasvir, asunaprevir, glecaprevir/pibrentasvir, sofosbuvir/velpatasvir, and sofosbuvir/velpatasvir/voxilaprevir are all administered at the same doses in patients with mild to moderate renal impairment (creatinine clearance $30-80 \mathrm{~mL} / \mathrm{min}$ ) as in those without kidney disease.

The safety and efficacy of those regimens have not been fully evaluated in patients with creatinine clearance $(\mathrm{CrCl})<30 \mathrm{~mL} /$ min, and sofosbuvir is not recommended for patients with $\mathrm{CrCl}$ $<30 \mathrm{~mL} / \mathrm{min}$ or those receiving dialysis.

In a study of genotype $1 \mathrm{HCV}$ patients with $\mathrm{CrCl}<30 \mathrm{~mL} / \mathrm{min}$ ( $n=20$, genotype 1a $65 \%$ ), regardless of dialysis, ombitasvir/paritaprevir/ritonavir and dasabuvir with ribavirin (200 mg/day, genotype 1a) or without ribavirin (genotype 1b) for 12 weeks resulted in an SVR12 of 90\% (18/20) without dose modification. ${ }^{152}$ Among genotype $1 \mathrm{HCV}$ patients with $\mathrm{CrCl}<30 \mathrm{~mL} / \mathrm{min}$ ( $\mathrm{n}=235,1 \mathrm{~b} 48 \%$, treatment naïve $80 \%$, cirrhosis $6 \%$ ), regardless of dialysis, elbasvir/grazoprevir for 12 weeks resulted in an SVR12 of $99 \%$ without dose modification. ${ }^{153}$ 
The Korean Association for the Study of the Liver (KASL) 2017 KASL clinical practice guidelines: Management of chronic hepatitis C

In patients with genotype 1-6 HCV infection and renal impairment of variable degrees, glecaprevir/pibrentasvir for 8-12 weeks without dose modification resulted in an SVR12 of $98 \%{ }^{154}$

Asunaprevir should be modified to $100 \mathrm{mg}$ daily in non-dialysis patients with $\mathrm{CrCl}<30 \mathrm{~mL} / \mathrm{min}$. No prospective study has examined the efficacy and safety of daclatasvir and asunaprevir for 24 weeks in genotype $1 \mathrm{~b}$ HCV-infected patients with renal impairment. In a retrospective Japanese study of dialysis patients, daclatasvir and asunaprevir for 24 weeks resulted in an SVR12 of 100\% without dose modification, and no significant adverse events were reported. ${ }^{155}$

Dose adjustment is needed depending on the severity of kidney disease because PegIFN- $a$ and ribavirin clearance are reduced by impaired kidney function. Moreover, ribavirin should be carefully used in patients with $\mathrm{CrCl}$ under $50 \mathrm{~mL} / \mathrm{min}$ because it can cause severe hemolytic anemia. ${ }^{156}$ Patients with mild kidney disease (glomerular filtration rate $(\mathrm{GFR}) \geq 60 \mathrm{~mL} / \mathrm{min}$ ) can receive the same doses of therapeutic drugs as those without kidney disease. If a patient has severe kidney disease (GFR of $15-59 \mathrm{~mL} / \mathrm{min}$ ), $135 \mu \mathrm{g}$ of PegIFN- $a-2 a$ or $1 \mu \mathrm{g} / \mathrm{kg}$ of PegIFN-a -2b along with 200$800 \mathrm{mg} /$ day of ribavirin twice a day with a gradual increase in dose is recommended. ${ }^{141}$ Patients on dialysis can take either interferon alpha or PegIFN-a, but the combination with ribavirin is not recommended. The SVR varied from 7-97\% in studies of combination therapy with PegIFN-a (135 $\mu$ g/week) and low-dose ribavirin (200 mg/day) in patients on dialysis, and most studies reported a high rate of treatment discontinuation.

Antiviral therapy for HCV can be conducted in patients with HCV-related cryoglobulinemia or membranous glomerulonephritis. Immunosuppressive therapy or plasma exchange can be performed prior to antiviral treatment in such patients with nephrotic syndrome or a rapid decrease in kidney function. ${ }^{157,158}$

In $41 \mathrm{HCV}$-infected patients with cryoglobulinemic vasculitis, sofosbuvir and daclatasvir were administered for 12-24 weeks, and immunologic laboratory results were compared between preand post-treatment. All the patients achieved SVR12, and 37 (90.2\%) patients showed complete resolution of purpura, arthralgia, and skin ulcers. The mean serum levels of cryoglobulin decreased from $0.56 \pm 0.18 \mathrm{~g} / \mathrm{dL}$ pre-treatment to $0.21 \pm 0.14 \mathrm{~g} / \mathrm{dL}$ at week 36 (SVR12-24), and in half of the patients, cryoglobulin became undetectable. ${ }^{159}$ In another study, 12 HCV-infected patients with cryoglobulinemia and systemic vasculitis underwent sofosbuvir-based antiviral therapy (concurrent rituximab therapy in 4 patients). The SVR12 was 83\%, and patients who achieved SVR12 showed improvement in their serum creatinine and a decrease of proteinuria. In $89 \%$ of patients, serum levels of cryoglobulin decreased after completion of the DAA therapy. ${ }^{160}$

\section{[Recommendations]}

1. Testing for anti-HCV antibodies should be performed in patients with chronic kidney disease who are under or planning for kidney replacement therapy, such as dialysis or kidney transplantation (B1).

2. HCV RNA should be tested to confirm HCV infection in patients with chronic kidney disease who have a positive anti-HCV antibody test or a negative anti-HCV antibody test but unknown liver disease (B1).

3. Sofosbuvir, ledipasvir/sofosbuvir, elbasvir/grazoprevir, ombitasvir/ paritaprevir/ritonavir with dasabuvir, daclatasvir, asunaprevir, glecaprevir/pibrentasvir, sofosbuvir/velpatasvir, and sofosbuvir/ velpatasvir/voxilaprevir can all be used without dose adjustment in patients with mild to moderate renal impairment (eGFR 30-80 $\mathrm{mL} / \mathrm{min}$ ) (B1).

4. Treatment of genotype 1-HCV infected patients with chronic hepatitis or compensated cirrhosis and severe renal impairment (eGFR $<30 \mathrm{~mL} / \mathrm{min})$

(1) Elbasvir/grazoprevir could be administered for 12 weeks without dose reduction (B1).

(2) Ombitasvir/paritaprevir/ritonavir and dasabuvir could be administered for 12 weeks without dose reduction. In genotype 1a infection, ribavirin (200 mg/day) is added (B1).

(3) Glecaprevir/pibrentasvir could be administered, and treatment duration is the same as in patients with normal eGFR (B1).

(4) Daclatasvir and asunaprevir could be administered for 24 weeks in genotype $1 \mathrm{~b}$ infection. In patients not on dialysis, the dose of asunaprevir needs to be reduced to $100 \mathrm{mg} /$ day (C1)

5. In genotype 2-6 HCV patients with chronic hepatitis or compensated cirrhosis and severe renal impairment (eGFR<30 $\mathrm{mL} / \mathrm{min}$ ), glecaprevir/pibrentasvir could be administered without modification of dose or treatment duration (B1).

6. In genotype $4 \mathrm{HCV}$ patients with chronic hepatitis or compensated cirrhosis and severe renal impairment (eGFR $<30 \mathrm{~mL} / \mathrm{min}$ ), elbasvir/grazoprevir could be considered for 12 weeks or ombitasvir/paritaprevir/ritonavir and dasabuvir with ribavirin (200 $\mathrm{mg} /$ day) could be considered for 12 weeks (C1).

7. In genotype 2, 3, 5, and 6 patients with eGFR less than $30 \mathrm{~mL} /$ min, PegIFN-a (135 $\mu \mathrm{g}$ of alpha-2a or $1 \mu \mathrm{g} / \mathrm{kg}$ of alpha-2b/wk) and ribavirin (200-800 mg/day) could be considered (B1).

In patients on hemodialysis, pegIFN- a (135 $\mu \mathrm{g}$ of alpha-2a/wk) without ribavirin could be considered (C2).

\section{Treatment of patients with HIV or HBV coinfection}

\section{Chronic hepatitis C patients with HIV coinfection}

The HIV and HCV coinfection rate is reported to be $25 \%$ in Western countries ${ }^{161}$ and $5.0-6.6 \%$ in South Korea. ${ }^{162,163}$ Because the frequency of coinfection is relatively high, all HIV-infected pa- 
tients should receive HCV testing, which consists primarily of an anti-HCV antibody assay. However, antibodies might not be present in $6 \%$ of HIV-infected patients, so an HCV RNA assay should be conducted in patients with idiopathic liver disease who are negative for anti-HCV antibodies. ${ }^{164}$ Chronic hepatitis C patients with risk factors for HIV infection should also be tested for HIV.

HIV-coinfected patients show rapid progress of liver disease, higher incidence of cirrhosis and mortality, and generally higher serum HCV RNA levels than those with HCV monoinfection. ${ }^{165-168}$ In particular, the progression of liver disease tends to accelerate with decreasing CD4+ lymphocyte counts and the deterioration of the immune system. ${ }^{169}$

In contrast, the recovery of immune function through antiretroviral therapy can delay the progression of liver disease by reducing HIV-related immune activation and inflammation. ${ }^{170-172}$ Therefore, antiretroviral therapy is generally recommended in HIV/HCV-coinfected patients regardless of their CD4+ lymphocyte count. However, antiretroviral therapy should be conducted carefully due to the high risk of liver toxicity caused by interactions between antiHCV and anti-HIV drugs, especially in HIV/HCV-coinfected patients with progressed liver disease. ${ }^{173,174}$ Antiretroviral therapy can be delayed in HIV treatment-naïve patients with a CD4+ lymphocyte count $>500 / \mathrm{mm}^{3}$ to prevent drug-drug interactions until HCV treatment is completed. In patients with a CD4 lymphocyte count $<200 / \mathrm{mm}^{3}$, antiretroviral therapy should be initiated promptly, but HCV therapy can be delayed until the patient is stable on HIV treatment because no data are available on the efficacy of DAA treatment in severely immunosuppressed patients. ${ }^{175,176}$

HIV/HCV-coinfected patients should be treated identically to HCV-monoinfected patients, and DAA treatment is recommended with priority because of the lower treatment efficacy of interferon-based regimens. When deciding on a regimen, considerations include drug-drug interactions with antiretroviral agents (Table 15), prior treatment history, and drug tolerance; moreover, expert consultation regarding HIV treatment is recommended. ${ }^{177}$

\section{Therapeutic agents:}

-Ribavirin: Anemia related to ribavirin is an increasingly important problem in treatment of HIV coinfection. It is more frequent and severe in patients taking zidovudine (AZT) and should therefore be avoided for those patients. ${ }^{178}$ Ribavirin can exacerbate didanosine (ddl) toxicity by inhibiting inosine-5-monophosphate dehydrogenase, and severe lactic acidosis, steatosis, and pancreatitis have been reported in patients taking $\mathrm{ddl}$ and ribavirin together; therefore, concomitant use of these two agents is contraindicat- ed. ${ }^{178-180}$ Patients receiving AZT and (especially) ddl should be switched to an equivalent antiretroviral agent before a combination therapy including ribavirin.

-Sofosbuvir: No clinically significant drug-drug interactions have been identified with most antiretroviral drugs (efavirenz, tenofovir dipivoxil fumarate [TDF], emtricitabine, rilpivirine, ritonavirboosted darunavir, and raltegravir), but co-administration with tipranavir is not recommended.

-Ledipasvir/sofosbuvir: When rilpivirine or efavirenz is used with TDF as an antiretroviral agent for treatment of HCV-HIV coinfection, TDF levels are increased by ledipasvir/sofosbuvir, which could increase the risk of renal toxicity. Concomitant use with ledipasvir/sofosbuvir in patients at high risk for renal toxicity (those with a $\mathrm{CrCl}$ of $30-60 \mathrm{~mL} / \mathrm{min}$ or pre-existing evidence of Fanconi syndrome) or those taking TDF and a ritonavir-boosted $\mathrm{Pl}$, should be monitored for potential renal injury by assessing renal function every $2-4$ weeks. ${ }^{159,181}$

-Daclatasvir: Dose adjustment of daclatasvir is not required when used with ritonavir-boosted darunavir or ritonavir-boosted lopinavir. The dose of daclatasvir should be reduced to $30 \mathrm{mg}$ once daily with ritonavir-boosted atazanavir and cobicistat-containing antiretroviral regimens, and an increased dose $(90 \mathrm{mg}$ daily) of daclatasvir is recommended when it is used with efavirenz or etravirine.

-Asunaprevir: Among antiretroviral agents, Pls are not recommended because the blood concentration of asunaprevir can be increased. Non-nucleoside reverse transcriptase inhibitors (NNRTIs) other than rilpivirine are not recommended because they can decrease the therapeutic effect of asunaprevir.

-Ombitasvir/paritaprevir/ritonavir and dasabuvir: Paritaprevir is an inhibitor of OATP1B1 and can increase indirect bilirubin. Ombitasvir/paritaprevir/ritonavir plus dasabuvir should only be used with antiretroviral drugs with which they do not interact: raltegravir, enfuvirtide, TDF, emtricitabine, lamivudine, atazanavir, and dolutegravir. Because ritonavir has anti-HIV activity, and lowdose ritonavir has the potential to select HIV resistance to $\mathrm{PI}$ in patients not undergoing antiretroviral therapy, HIV/HCV-coinfected patients should achieve HIV RNA suppression prior to initiation of this regimen. In particular, because this combination contains $100 \mathrm{mg}$ of ritonavir, the dose of ritonavir used to boost HIV PIs 
The Korean Association for the Study of the Liver (KASL) 2017 KASL clinical practice guidelines: Management of chronic hepatitis C

Table 15. Concomitant use of HIV and HCV drugs ${ }^{\dagger+}$

\begin{tabular}{|c|c|c|c|c|c|c|c|c|c|}
\hline Co-medications & SOF & $\begin{array}{l}\text { LDV/ } \\
\text { SOF }\end{array}$ & DCV & ASV & $\begin{array}{l}\mathrm{EBR} / \\
\mathrm{GZR}\end{array}$ & OPr-D & $\begin{array}{l}\text { SOF/ } \\
\text { VEL }\end{array}$ & $\begin{array}{c}\text { SOF/ } \\
\text { VEL/VOX }\end{array}$ & $\mathrm{G} / \mathrm{P}$ \\
\hline \multicolumn{10}{|l|}{ Nucleoside analogue reverse transcriptase inhibitors (NRTIs) } \\
\hline Abacavir & O & 0 & 0 & NA & 0 & O & 0 & NA & 0 \\
\hline Emtricitabine & 0 & 0 & 0 & NA & 0 & 0 & 0 & 0 & 0 \\
\hline Lamivudine & 0 & 0 & O & NA & 0 & O & 0 & NA & 0 \\
\hline Stavudine & 0 & 0 & 0 & NA & 0 & 0 & 0 & NA & 0 \\
\hline Tenofovir disoproxil fumarate & 0 & $\Delta^{*}$ & 0 & NA & 0 & 0 & $\Delta^{*}$ & $\Delta^{*}$ & 0 \\
\hline Zidovudine & 0 & 0 & 0 & NA & 0 & 0 & 0 & NA & 0 \\
\hline \multicolumn{10}{|l|}{$\begin{array}{l}\text { Non-nucleoside analogue reverse transcriptase inhibitors } \\
\text { (NNRTIs) }\end{array}$} \\
\hline Efavirenz & 0 & $\Delta^{\dagger}$ & $\Delta^{\S}$ & $x$ & $x$ & $x$ & $x$ & $x$ & $x$ \\
\hline Etravirine & 0 & 0 & $\Delta$ & $x$ & $x$ & $x$ & $x$ & NA & $x$ \\
\hline Nevirapine & 0 & 0 & $\Delta$ & $x$ & $x$ & $x$ & $x$ & NA & $x$ \\
\hline Rilpivirine & 0 & 0 & 0 & 0 & 0 & $\Delta$ & 0 & 0 & 0 \\
\hline \multicolumn{10}{|l|}{ Protease inhibitors (PIs) } \\
\hline Atazanavir & 0 & 0 & $\Delta^{\|}$ & $x$ & $x$ & $\Delta^{* *}$ & 0 & $x$ & $x$ \\
\hline Darunavir & 0 & 0 & 0 & $x$ & $x$ & $x$ & 0 & 0 & $x$ \\
\hline Fosamprenavir & 0 & 0 & $\Delta^{\|}$ & $x$ & $x$ & $\Delta$ & 0 & NA & $x$ \\
\hline Lopinavir & 0 & $\Delta^{\ddagger}$ & 0 & $x$ & $x$ & $x$ & 0 & $x$ & $x$ \\
\hline Saquinavir & 0 & 0 & $\Delta^{\|}$ & $x$ & $x$ & $x$ & 0 & NA & $x$ \\
\hline Tipranavir & $x$ & $x$ & $\Delta^{\|}$ & $x$ & $x$ & $x$ & $x$ & $x$ & $x$ \\
\hline \multicolumn{10}{|l|}{ Pharmacokinetic enhancers } \\
\hline Ritonavir & 0 & $\Delta$ & 0 & $x$ & $x$ & $x$ & 0 & 0 & $x$ \\
\hline Cobicistat (with darunavir) & 0 & $\Delta$ & $\Delta$ & $x$ & $x$ & $x$ & 0 & 0 & $\Delta^{* *}$ \\
\hline Cobicistat (with atazanavir) & & & & & & & & $x$ & \\
\hline \multicolumn{10}{|l|}{ Integrase inhibitors } \\
\hline Dolutegravir & 0 & 0 & 0 & NA & 0 & O & 0 & 0 & 0 \\
\hline Raltegravir & 0 & 0 & 0 & NA & 0 & 0 & 0 & 0 & 0 \\
\hline \multicolumn{10}{|l|}{ Entry inhibitor } \\
\hline Maraviroc & 0 & $\Delta$ & 0 & NA & 0 & $\Delta$ & O & NA & 0 \\
\hline \multicolumn{10}{|l|}{ Combinations } \\
\hline $\begin{array}{l}\text { Elvitegravir/cobicistat/emtricitabine/tenofovir disoproxil } \\
\text { fumarate }\end{array}$ & 0 & $\Delta$ & $\Delta^{\|}$ & NA & $x$ & $x$ & $\Delta$ & $\Delta$ & 0 \\
\hline Elvitegravir/cobicistat/emtricitabine/tenofovir alafenamide & 0 & 0 & $\Delta^{\|}$ & NA & $x$ & $x$ & 0 & 0 & 0 \\
\hline
\end{tabular}

HIV, human immunodeficiency virus; HCV, hepatitis C virus; SOF, sofosbuvir; LDV, ledipasvir; DCV, daclatasvir; ASV, asunaprevir; EBR, elbasvir; GZR, grazoprevir; OPr-D, ombitasvir/paritaprevir/ritonavir plus dasabuvir; VEL, velpatasvir; VOX, voxilaprevir; G, glecaprevir; P, pibrentasvir; NA, not available; O, no clinical significant interaction expected; $X$, these drugs should not be co-administered; $\Delta$, potential interaction that might require dose adjustment, altered timing of administration, or additional monitoring.

*Monitor for tenofovir toxicity; ${ }^{\dagger}$ If $\mathrm{PI} / \mathrm{r}$ (or atazanavir/r, darunavir/c) is used with tenofovir, increase of tenofovir concentrations are expected. If coadministration necessary, monitor for tenofovir-associated toxicities; "If efavirenz used with tenofovir/emtricitabine, monitor for tenofovir toxicity due to increase of tenofovir

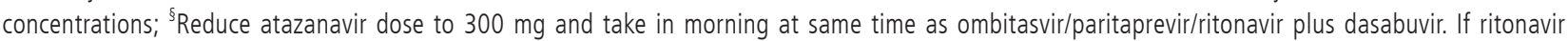
cannot be used, choose an alternative HCV regimen; "Take atazanavir $300 \mathrm{mg}$ in morning at same time as ombitasvir/paritaprevir/ritonavir plus dasabuvir; discontinue ritonavir or cobicistat in HIV regimen until HCV therapy completed; ${ }^{* *}$ Coadministration of glecaprevir/pibrentasvir and cobicistat (with elvitegravir/ emtricitabine/tenofovir alafenamide) increased glecaprevir Cmax, area under the curve and $\mathrm{Cmin}$ by 2.50-fold, 3.05-fold and 4.58-fold, respectively. However these increases were deemed to be within safety limits. Coadministration with cobicistat-boosted HIV integrase inhibitors were allowed in clinical studies, however, cobicistat-boosted HIV protease inhibitors are not recommended (see individual HIV protease inhibitors for recommendations); ${ }^{\dagger \dagger}$ Presenting information is based on the data available until August 2017. 
might need to be adjusted (or held) when administered with ombitasvir/paritaprevir/ritonavir plus dasabuvir and then restored upon completion of HCV treatment. Concomitant use of efavirenz, rilpivirine, and darunavir with ritonavir-boosted lopinavir is not recommended. When that combination is used with ribavirin, unboosted HIV PIs, rilpivirine, and efavirenz should not be used. When this combination is used with efavirenz, emtricitabine, or TDF, gastrointestinal and neurologic adverse events and elevations of ALT can occur. ${ }^{159,182}$

-Elbasvir/grazoprevir: It is not recommended to use elbasvir/ grazoprevir concomitantly with any ritonavir-boosted HIV PI and efavirenz. Although no study has considered the interaction between etravirine or cobicistat-boosted elvitegravir and elbasvir/ grazoprevir, concomitant use of these drugs should be avoided. Elbasvir/grazoprevir can be used concomitantly with raltegravir, dolutegravir, rilpivirine, and HIV nucleoside analogues.

-Glecaprevir/pibrentasvir: It is not recommended to use glecaprevir/pibrentasvir concomitantly with any PI and efavirenz. Although no study has considered the interaction between etravirine or nevirapine and glecaprevir/pibrentasvir, concomitant use of these drugs should be avoided. Glecaprevir/pibrentasvir can be used concomitantly with all NRTIs, integrase inhibitor, elvitegravir/ cobicistat/emtricitabine/TDF, elvitegravir/cobicistat/emtricitabine/ tenofovir alafenamide (TAF), and rilpivirine.

-Sofosbuvir/velpatasvir: Like ledipasvir/sofosbuvir, sofosbuvir/velpatasvir increases serum tenofovir levels. Thus, co-administration of sofosbuvir/velpatasvir and TDF can cause problems in patients with $\mathrm{CrCl}$ less than $60 \mathrm{~mL} / \mathrm{min}$ and in those receiving an anti-HIV regimen that contains ritonavir or cobicistat. Use of TAF instead of TDF is recommended, and renal function should be monitored if TDF is used. Efavirenz decreases serum velpatasvir levels, so it is not recommended to use those drugs concomitantly. Etravirine and sofosbuvir/velpatasvir are also not recommended for concomitant use, although no study has considered their drug-drug interaction. It was reported that concomitant use of atazanavir/ritonavir with sofosbuvir/velpatasvir elevated serum indirect bilirubin levels. However, the clinical relevance of that finding is unclear.

-Sofosbuvir/velpatasvir/voxilaprevir: Co-administration of atazanavir and voxilaprevir should be avoided because atazanavir increases the serum levels of voxilaprevir. Tipranavir decreases the serum levels of sofosbuvir and velpatasvir, and efavirenz decreases the levels of velpatasvir and voxilaprevir. Thus, concomitant use of those drugs is not recommended. Monitoring of renal function is required when a TDF-containing HIV regimen is co-administered with sofosbuvir/velpatasvir/voxilaprevir.

\section{Treatment efficacy:}

In 50 treatment-naïve patients with HCV genotype 1 and HIV co-infection without cirrhosis, the SVR was $98 \%$ after 12 weeks of ledipasvir/sofosbuvir treatment. ${ }^{181}$ After 12 weeks of ledipasvir/ sofosbuvir treatment, 335 patients coinfected with HIV and genotype 1 or $4 \mathrm{HCV}$ who had been previously treated or untreated for HCV (cirrhosis 20\%), showed an SVR of 96\%. ${ }^{183}$

In 63 patients with HCV genotype 1 and HIV co-infection who were HCV treatment-naïve or had a history of prior treatment failure (including cirrhotic patients), the SVR was $91-94 \%$ after 12 or 24 weeks of ombitasvir/paritaprevir/ritonavir plus dasabuvir and ribavirin. ${ }^{182}$ After 12 weeks of daclatasvir plus sofosbuvir treatment in 153 previously untreated or treated patients with HIV/ HCV coinfection (genotypes 1-4), the SVR was $97-98 \% .{ }^{30}$ In 59 non-cirrhotic patients with HCV genotype 1 and HIV co-infection, the SVR was $87 \%$ and $97 \%$ after 12 weeks of elbasvir/grazoprevir without ribavirin and with ribavirin, respectively. ${ }^{70}$ In an open-label, phase III study of 106 patients (cirrhosis 18\%) with HCV genotype 1-4 and HIV co-infection, the overall SVR was $95 \%$ (101/106) after sofosbuvir/velpatasvir for 12 weeks: 95\% (74/78) in genotype $1 \mathrm{~b}, 100 \%$ (11/11) in genotype $2,92 \%(11 / 12)$ in genotype 3 , and $100 \%(5 / 5)$ in genotype $5 .{ }^{105}$ In 137 non-cirrhotic patients with HCV genotype 1-6 and HIV co-infection, 8 weeks of glecaprevir/pibrentasvir resulted in an SVR of $100 \%$. In 16 cirrhotic patients with genotype 1-6 and HIV co-infection, the SVR rate was $93 \%$ after 12 weeks of glecaprevir/pibrentasvir.

In patients with genotype 2 or 3 infection, including those with compensated cirrhosis, sofosbuvir plus weight-based ribavirin treatment for 12 weeks in 68 treatment-naïve patients and 24 weeks in 41 retreatment patients resulted in SVR rates of $88 \%$ for genotype 2 and $67 \%$ in genotype 3 patients. ${ }^{68}$ Using the same regimen, 163 HIV-coinfected patients (including cirrhotic patients) were treated for 12 weeks (treatment-naive patients with genotype 2 infection) or 24 weeks (genotype 3 or 4 and retreated genotype 2 patients); the resulting SVR rates were $88 \%$ for genotype 2, 89\% for genotype 3, and $84 \%$ for genotype 4 patients. ${ }^{184}$

Data regarding retreatment of HIV-coinfected patients and retreatment of sofosbuvir-experienced patients in that population are insufficient. 
The Korean Association for the Study of the Liver (KASL) 2017 KASL clinical practice guidelines: Management of chronic hepatitis C

\section{[Recommendations]}

1. All HIV infected patients should be tested for anti-HCV antibodies (B1).

2. HCV RNA assays should be conducted in HIV infected patients positive for anti-HCV antibodies or negative for anti-HCV antibodies but with unknown liver disease (B1).

3. Antiretroviral treatment interruption to allow HCV therapy is not recommended (B1).

4. Interferon-free DAA treatment is a priority recommendation for HIV/HCV-coinfected patients, who should be treated the same as HCV-monoinfected patients using DAA for which information on drug interactions with antiretroviral agents is available (B1).

5. When HIV/HCV-coinfected patients are treated with DAAs, drug-drug interactions should be carefully considered, and consultation with an HIV treatment expert is recommended if the antiretroviral therapy regimen is to be modified (A1).

\section{Chronic hepatitis C patients with HBV coinfection}

The number of HBV/HCV coinfected patients is estimated to be $15,000,000$ worldwide, ${ }^{185}$ and $2.37 \%$ of anti-HCV positive patients are reported to be coinfected with HBV in South Korea. ${ }^{186}$

A 10-year follow-up study of HCV-monoinfected patients reported an HCC occurrence rate of $28 \%$, whereas HCV/HBV-coinfected patients showed an occurrence rate of $45 \%$, which is significantly higher. ${ }^{187}$ In addition, risks of severe and fulminant hepatitis increase, along with the incidence of cirrhosis and HCC, in HBV/HCV-coinfected patients compared to those with HBV monoinfection. ${ }^{188,189}$

In patients with HBV/HCV coinfection, blood HCV RNA and HBV DNA levels, which are indicators of the replicative status of each virus, should be evaluated. If HCV infection is the dominant cause of liver disease, the same antiviral therapy as for HCV monoinfec- tion is recommended. Indeed, the SVR following PegIFN- $a$ and ribavirin is similar to that in HCV monoinfection. ${ }^{190,191}$

Treatment of patients with HBV/HCV coinfection is identical to that of HCV-monoinfected patients. The risk of drug-drug interactions between DAAs and anti-HBV agents is low, with the exception of asunaprevir, for which information is lacking. Renal function monitoring is warranted if ledipasvir is used with tenofovir because renal toxicity can be increased (Table 16). Reactivation of HBV is possible during or after HCV treatment, ${ }^{187,192}$ and administration of oral antiviral agents could be indicated if significant proliferation of HBV is confirmed. ${ }^{193}$

In a retrospective study conducted in the United States (US), the reactivation of $\mathrm{HBV}$ during or after DAA therapy was defined as a $1,000 \mathrm{IU} / \mathrm{mL}$ increase in HBV DNA or hepatitis B surface antigen (HBsAg) detection in a person who was previously negative for HBsAg. Nine of 62,290 patients treated with DAAs had evidence of $\mathrm{HBV}$ reactivation during DAA treatment. HBV reactivation occurred in $8 \mathrm{HBsAg}$-positive patients and one isolated anti-HBCpositive patient. Seventeen other patients had small increases in HBV DNA levels that did not qualify as HBV reactivation. Only 3 of the 9 patients with HBV reactivation in this cohort exhibited peak alanine aminotransferase elevations $\geq 2$ times the upper limit of normal. ${ }^{194}$ In contrast, cases reported to the US Food and Drug Administration (FDA) Adverse Event Reporting System include patients with serious HBV reactivation after DAA treatment. ${ }^{195}$ That is, the FDA identified 29 unique reports of HBV reactivation in patients receiving DAAs from 22 November 2013 to 15 October 2016. Two cases resulted in death and 1 case in liver transplantation. Patients in whom HBV reactivation developed were heterogeneous regarding HCV genotype, DAAs received, and baseline

Table 16. Concomitant use of HBV and HCV drugs*

\begin{tabular}{|c|c|c|c|c|c|c|c|c|c|c|c|}
\hline & \multicolumn{11}{|c|}{ HCV drugs } \\
\hline & SOF & LDV/SOF & DCV & ASV & EBR/GZR & OPr-D & SOF/VEL & SOF/VEL/VOX & $\mathrm{G} / \mathrm{P}$ & PEG-IFN & RBV \\
\hline \multicolumn{12}{|l|}{ HBV drugs } \\
\hline Adefovir & 0 & 0 & 0 & NA & 0 & 0 & 0 & NA & 0 & $\Delta$ & NA \\
\hline Entecavir & 0 & 0 & 0 & NA & 0 & 0 & 0 & NA & 0 & NA & NA \\
\hline Lamivudine & 0 & 0 & 0 & NA & 0 & 0 & 0 & NA & 0 & $\Delta$ & $\Delta$ \\
\hline Telbivudine & 0 & 0 & 0 & NA & 0 & 0 & 0 & NA & 0 & $x$ & NA \\
\hline Tenofovir & 0 & $\Delta$ & 0 & NA & 0 & 0 & $\Delta$ & $\Delta$ & 0 & $\Delta$ & $\Delta$ \\
\hline
\end{tabular}

HBV, hepatitis B virus; HCV, hepatitis C virus; SOF, sofosbuvir; LDV, ledipasvir; DCV, daclatasvir; ASV, asunaprevir; EBR, elbasvir; GZR, grazoprevir; OPr-D, ombitasvir/paritaprevir/ritonavir plus dasabuvir; VEL, velpatasvir; VOX, voxilaprevir; G, glecaprevir; P, pibrentasvir; PEG-INF, pegylated interferon; RBV, ribavirin; 0 , no clinical significant interaction expected; $\triangle$, potential interaction, may require close monitoring, alteration of drug dosage or timing of administration; NA, data not available; $X$, these drugs should not be coadministered.

*Presenting information is based on the data available until August 2017. 
HBV characteristics. At baseline, 9 patients had a detectable HBV viral load, 7 had positive results on HBsAg testing and an undetectable HBV viral load, and 3 had negative results on $\mathrm{HBsAg}$ testing and an undetectable HBV viral load. For the remaining 10 patients, data points were not reported or the data were uninterpretable. Caution needs to be paid because no risk factors related to HBV reactivation during or after DAA therapy have been identified. Therefore, serologic tests such as HBsAg, anti-HBs, and anti$\mathrm{HBC}$ are recommended before DAA therapy to determine whether a patient has HCV and HBV co-infection. ${ }^{196}$

\section{[Recommendations]}

1. After confirming the dominant cause of liver disease in HBV/HCV coinfection, treatment following the same rules as monoinfection is recommended, and periodic HBV DNA quantitation is recommended during and after antiviral therapy for HCV infection (B1).

2. In patients with HBV/HCV coinfection, oral administration of anti$\mathrm{HBV}$ agents could be indicated if significant proliferation of HBV is confirmed during or after antiviral therapy for HCV infection (B1).

\section{Hemophilia or thalassemia}

An HCV infection in patients with hemophilia or thalassemia causes significant increases in morbidity and mortality rates compared with patients without HCV infection. ${ }^{197-200}$ Therefore, aggressive treatment of $\mathrm{HCV}$ infection should be considered.

Hemophilia A and B, caused by a deficiency of factor VIII and $I X$, respectively, can increase a patient's chance of being exposed to HCV due to multiple transfusions necessitated by spontaneous and traumatic bleeds. Coinfection with HIV/HCV is not a contraindication to liver transplantation in hemophilia, and indications for liver transplantation in patients with hemophilia are the same as those in non-hemophilic individuals. ${ }^{111}$ A randomized, placebocontrolled, phase 3 study of 159 adults with HCV infection and sickle cell anemia, thalassemia, or hemophilia A/B or von Willebrand disease evaluated the safety and efficacy of elbasvir/grazoprevir for 12 weeks. One hundred of the 107 patients (93.5\%) achieved SVR12; 6 relapsed, and 1 was lost to follow-up. SVR12 was achieved in $94.7 \%$ (18 of 19), 97.6\% (40 of 41), and $89.4 \%$ (42 of 47) of patients with sickle cell disease, $\beta$-thalassemia, and hemophilia A/B or von Willebrand disease, respectively. Hemoglobin levels and international normalized ratio values were similar in patients receiving elbasvir/grazoprevir and placebo. Among patients with hemoglobinopathies, change in mean hemoglobin levels were similar in those receiving elbasvir/grazoprevir and those receiving placebo. ${ }^{201}$ Another study evaluated the safety and efficacy of ledipasvir/sofosbuvir and sofosbuvir plus ribavirin in 120 patients with HCV genotype 1-4 infection and an inherited bleeding disorder. Ledipasvir/sofosbuvir was administered for 12 weeks to patients with genotype 1 or 4 infection and for 12 or 24 weeks to treatment-experienced cirrhotic patients with genotype 1 infection. Patients with genotype 2 and 3 infection received sofosbuvir plus ribavirin for 12 and 24 weeks, respectively. Overall, $65 \%$ of patients had hemophilia $A$ and $26 \%$ of patients had hemophilia B; 22\% were HIV coinfected. SVR12 was achieved in $99 \%$ (98/99) of patients with genotype 1 or 4 infection, 100\% $(5 / 5)$ in treatment-experienced cirrhotic patients with genotype 1 infection; $100 \%(10 / 10)$ in patients with genotype 2 infection; and $83 \%$ (5/6) in patients with genotype 3 infection. No treatment discontinuations occurred due to adverse events. Bleeding occurred in 22 patients, but only one was considered to be related to the treatment. ${ }^{202}$

Severe anemia can occur due to ribavirin in thalassemia, and $30-40 \%$ of cases can require blood transfusion at 3-4 week intervals to maintain hemoglobin at 9-10 $\mathrm{g} / \mathrm{dL}$. Therefore careful monitoring for hematological complications is required. However, the frequency of treatment discontinuation and the incidence of other main complications did not increase in those patients. ${ }^{197}$

\section{[Recommendations]}

1. Patients with hemophilia should be treated following the same rules as persons without bleeding disorders (A1).

2. Patients with thalassemia should be treated following the same rules as persons without hemoglobinopathy (B1)

\section{Patients receiving immunosuppressants or cytotoxic chemotherapy}

Although one study defined HCV reactivation as the re-emergence of or increase in HCV RNA plus elevation of ALT up to threefold the upper limit of normal, ${ }^{202}$ there is no universal consensus on the definition of HCV reactivation; increases in blood ALT and HCV RNA levels are commonly used as the criteria.

The incidence of HCV reactivation in patients taking immunosuppressants or under cytotoxic chemotherapy is lower than that of $\mathrm{HBV} .^{202-205}$ For example, the reactivation rate of $\mathrm{HCV}$ was $0 \%$ (0/11) compared to $38 \%(3 / 8)$ for HBV in a study including 98 non-Hodgkin's lymphoma patients receiving chemotherapy. ${ }^{206}$ However, another study of B cell non-Hodgkin's lymphoma re- 
The Korean Association for the Study of the Liver (KASL) 2017 KASL clinical practice guidelines: Management of chronic hepatitis C

ported a higher incidence ( $26.3 \%$ vs. $2.1 \%$ ) of significant ALT elevation in HCV-infected patients compared to patients without HCV infection, indicating that HCV reactivation does occur and can cause clinically significant morbidity. ${ }^{207}$

Risk factors for HCV reactivation have not been clearly identified. However, reactivation has been reported to occur more frequently in patients with hematological malignancies. ${ }^{203,208} \mathrm{HCV}$ reactivation has also been reported in patients with solid cancers and in those who underwent stem cell transplantation. ${ }^{209,210} \mathrm{Al}$ though death due to HCV reactivation is rare, ${ }^{211}$ the mortality is similar to that of HBV once severe hepatitis occurs due to HCV reactivation. ${ }^{212,213}$

Strategies to prevent HCV reactivation in these patients have not been established. Conservative therapy and discontinuation of the offending drugs are currently recommended. However, both morbidity from HCV reactivation and the disadvantages of immunosuppressive drug discontinuation should be taken into account, and decisions should be individualized. Further studies are needed to explore whether DAAs might have a benefit in preventing and treating HCV reactivation during immunosuppressive treatment or cytotoxic chemotherapy.

\section{Children}

In a Korean study involving 2,080 6 to 11 year-old children in 1996, the anti-HCV-antibody positive rate was $0.82 \% .{ }^{214}$ The transfusion of infected blood components or vertical transmission is the most common cause of HCV infection in children, ${ }^{215}$ although transfusion-related HCV transmission has been rarely reported since the introduction of screening for HCV infection in 1991 in South Korea. The global HCV infection rate among pregnant women has been reported to be $0.49-1.7 \%{ }^{216,217} \mathrm{~A}$ Korean study involving 5,000 pregnant women and another study with 20,000 pregnant women reported anti-HCV-antibody positivity rates of $0.42-0.44 \%$, with $57-60 \%$ of anti-HCV positive pregnant women also positive for HCV RNA. ${ }^{218,219}$

The frequency of HCV transmission was reported to be 1-6.2\% during the perinatal period, ${ }_{1}^{157,220}$ and the evidence that Cesarean section reduces the risk of vertical HCV transmission is weak. ${ }^{221}$ Although HCV RNA has been detected in human milk, the acquisition of HCV infection from human milk has not been documented. Horizontal infections among children are rare, so there is no need to limit routine activities, such as school life or exercise. ${ }^{221}$

An anti-HCV assay in children is recommended after 18 months of age because maternal antibodies can be delivered to new- borns. ${ }^{221,222}$ An HCV RNA assay can be performed at 1 or 2 months of age if earlier diagnosis is desired, although the sensitivity is as low as 22\% at that time; therefore, HCV RNA assays should be conducted at an age older than 6 months, when the sensitivity reaches $85 \%{ }^{222,223}$

Spontaneous recovery is more frequent in children than in adults and tends to show a normal ALT level. ${ }^{224}$ In addition, HCV infection in children often shows slow progression of hepatic fibrosis and only rarely results in severe hepatic damage. However, aggressive treatment during childhood has been suggested because children usually have a regular lifestyle and show higher therapeutic compliance than adults. Aggressive treatment is considered in cases of continuously elevated serum AST/ALT levels or when advanced hepatic fibrosis is confirmed by liver biopsy. In addition, treatment can be also considered even in cases of normal serum AST/ALT levels or mild fibrosis on liver biopsy because the tools to predict disease progression are insufficient in children. $^{225}$

Few data on DAA treatment are available in children. A phase 2, multicenter, open-label study of 100 adolescents aged 12-17 years (mean 15 years) with genotype $1 \mathrm{HCV}$ infection assessed the safety and efficacy of 12 weeks of ledipasvir/sofosbuvir. ${ }^{226}$ Most participants (80\%) were treatment-naïve, and $84 \%$ were infected through perinatal transmission. Overall, 98\% (98/100) of patients achieved SVR12, and the 2 patients who did not achieve SVR2 were lost to follow-up either during or after treatment. The three most common adverse events were headache (27\%), diarrhea (14\%), and fatigue (13\%). No serious adverse events were reported. The area under the concentration-time curve (tau) and maximum concentration values for sofosbuvir, ledipasvir, and GS331007 were within the predefined pharmacokinetic equivalence boundaries of $50 \%-200 \%$ when compared with adults from phase 2 and 3 studies of ledipasvir and sofosbuvir.

Although HCV-infected children were treated with interferon-a monotherapy due to the potential teratogenic effects of ribavirin in previous studies, higher SVR rates have recently been reported with the addition of ribavirin. ${ }^{227-229}$ Therefore, most studies have adopted combination therapy for children because that approach is standard in adults. The use of PegIFN-a in children older than 3 years of age has been approved in North America and Europe. ${ }^{225}$

The dose of PegIFN-a 2a is $180 \mu \mathrm{g} / 1.73 \mathrm{~m}^{2} /$ week, and the dose of ribavirin is $15 \mathrm{mg} / \mathrm{kg}$ twice a day. Genotype 1 and 4 patients should be treated for 48 weeks, and genotype 2 and 3 patients should be treated for 24 weeks, similar to adults. ${ }^{225}$ The SVR after combination therapy of PegIFN-a and ribavirin (47-53\% in geno- 
type 1 and $80-100 \%$ in genotypes 2 and 3) is superior to that of combination therapy with interferon alpha and ribavirin. ${ }^{227-229}$ Factors that predict an SVR include infection by genotypes 2 and 3 and an HCV RNA titer $<600,000 \mathrm{IU} / \mathrm{mL}$. $^{228,229}$

\section{[Recommendations]}

1. Diagnosis and evaluation of HCV in children should proceed following the same rules as in adults (B1).

2. An anti-HCV assay for children is recommended at age $>18$ months because maternal antibodies can be delivered to newborns. If an earlier assay is required, an HCV RNA assay can be considered after 6 months of age (B2).

3. HCV infected children aged 3-17 years should be considered appropriate candidates for treatment according to the same criteria used in adults (B1).

4. The dose of PeglFN-a 2a is $180 \mu \mathrm{g} / 1.73 \mathrm{~m}^{2} /$ week, and the dose of ribavirin is $15 \mathrm{mg} / \mathrm{kg} /$ day. Genotype 1 and 4 patients should be treated for 48 weeks, and genotype 2 and 3 patients should be treated for 24 weeks (B1).

\section{RETREATMENT OF PATIENTS WITH DAA TREATMENT FAILURE}

Several clinical trials have reported the results of retreatment in patients who failed in prior DAA treatment. As more effective DAAs and new data from further clinical studies are released, the following recommendations could be changed.

The SVR of 70\% (38/54) was achieved after a 12-week retreatment with ledipasvir/sofosbuvir in 54 genotype $1 \mathrm{~b}$ CHC patients who failed with a 24-week daclatasvir and asunaprevir treatment. ${ }^{230}$ According to the types of previous treatment failure, the SVR were 18\% (2/11), 69\% (11/16), 94\% (15/16), and 100\% (7/7) in patients with non-response, viral breakthrough, relapse, and discontinuation due to adverse events, respectively. The SVR $(0 \%$, 0/8) was significantly lower in patients with a FIB4 index of $\geq 3.25$ who had not responded to prior treatment, compared with the others $(83 \%, 35 / 42)$.

Forty-one genotype 1 CHC patients (34 genotype 1a, 7 genotype 1b; 19 liver cirrhosis) who relapsed following 8 or 12 weeks of ledipasvir/sofosbuvir treatment were retreated with ledipasvir/ sofosbuvir for 24 weeks. ${ }^{231}$ The overall SVR was 71\% (29/41). The SVR of the patients who failed following 8 weeks of treatment was $80 \%(24 / 30)$, which was higher than the $45 \%(5 / 11)$ of patients who failed following 12 weeks of treatment. The SVR in 30 patients with baseline RASs was 60\% (18/30), and that of 11 pa- tients without RASs was $100 \%$ (11/11). The SVR was $69 \%$ with one RAS and $50 \%$ with two or more RASs. The SVR of patients with NS5A RASs (ex. Y93H/N) was 33\% (2/6), and that of patients with NS5B RASs (ex. S282T) was 25\% (3/12).

Twenty-five genotype 1 CHC patients (22 genotype 1a, 3 genotype $1 \mathrm{~b} ; 5$ cirrhosis) who failed with a short-term combination therapy of sofosbuvir and elbasvir/grazoprevir for 4, 6, or 8 weeks were retreated with sofosbuvir, elbasvir/grazoprevir and ribavirin for 12 weeks. ${ }^{232}$ The overall SVR was 100\% (25/25), including all patients with baseline NS3 RASs (17 patients) and NS5A RASs (14 patients).

Twenty-two genotype 1 CHC patients (20 genotype 1a, 2 genotype $1 \mathrm{~b} ; 6$ cirrhosis) who failed with previous DAA treatments (ombitasvir/paritaprevir/ritonavir and dasabuvir 14, ombitasvir/ paritaprevir/ritonavir 2) were retreated with sofosbuvir, ombitasvir/paritaprevir/ritonavir, and dasabuvir with or without ribavirin. ${ }^{233}$ The overall SVR was $95 \%$ (21/22). In genotype 1a patients without cirrhosis, the SVR following treatment with sofosbuvir, ombitasvir/paritaprevir/ritonavir, dasabuvir, and ribavirin for 12 weeks was 92\% (13/14), and in genotype 1a patients with cirrhosis, the SVR from the same regimen for 24 weeks was 100\% (7/7). The SVR following sofosbuvir, ombitasvir/paritaprevir/ritonavir, and dasabuvir for 12 weeks in genotype $1 \mathrm{~b}$ patients was 100\% (2/2). All 18 patients with baseline RASs achieved SVR.

A total of 263 CHC patients (101 genotype 1a, 45 genotype 1b, 4 other genotype 1, 5 genotype 2, 78 genotype 3, 22 genotype 4, 1 genotype 5, 6 genotype 6, 1 unknown; 121 cirrhosis) who failed in previous DAA treatments containing a NS5A inhibitor (NS5A and NS5B inhibitor 161, NS5A and NS3 inhibitor 83, NS5A inhibitor 18) were retreated with sofosbuvir/velpatasvir/voxilaprevir for 12 weeks. The overall SVR was $96 \%(253 / 263){ }^{234}$ The SVR of patients with genotype 1a and $1 \mathrm{~b}$ were $96 \%(97 / 101)$ and $100 \%$ (45/45), respectively. The SVR of patients with genotype 2, 3, 4, 5, and 6 were 100\% (5/5), 96\% (74/78), 91\% (20/22), 100\% (1/1), and $100 \%(6 / 6)$, respectively. The SVR of patients without baseline RASs was 98\% (42/43), and the SVR of patients with RASs was $97 \%$ (199/205). The SVR of the 121 patients with cirrhosis was $93 \%(113 / 121)$.

In total, 333 CHC patients (98 genotype 1a, 46 genotype 1b, 64 genotype 2, 106 genotype 3, 19 genotype 4; 153 cirrhosis) who failed with previous DAA treatment without a NS5A inhibitor (NS5B inhibitors 243, NS5 inhibitors 84, NS3 inhibitors 5) were retreated with sofosbuvir/velpatasvir/voxilaprevir or sofosbuvir/ velpatasvir for 12 weeks. ${ }^{234}$ The overall SVR was 98\% (178/182) with sofosbuvir/velpatasvir/voxilaprevir and 90\% (136/151) with 
The Korean Association for the Study of the Liver (KASL) 2017 KASL clinical practice guidelines: Management of chronic hepatitis C

sofosbuvir/velpatasvir. By genotype, sofosbuvir/velpatasvir/voxilaprevir and sofosbuvir/velpatasvir showed SVR of $98 \%(53 / 54)$ and $89 \%$ (39/44) for genotype 1a, 96\% (23/34) and 85\% (21/22) for genotype $1 \mathrm{~b}, 100 \%$ (31/31) and $97 \%$ (32/33) for genotype 2, and $96 \%(52 / 54)$ and $85 \%$ (44/52) for genotype 3, respectively. All 19 patients with genotype 4 were treated with sofosbuvir/velpatasvir/voxilaprevir for 12 weeks, and their SVR was 100\% (19/19). The SVR was 89\% (67/75) without NS3 or NS5A baseline RASs, $90 \%(63 / 70)$ with NS3 or NS5A baseline RASs, and 50\% (2/4) with both NS3 and NS5A baseline RASs following 12 weeks of sofosbuvir/velpatasvir treatment. All 83 patients with NS3 or NS5A baseline RASs who received sofosbuvir/velpatasvir/voxilaprevir for 12 weeks achieved SVR.

Sixty-nine CHC patients (32 genotype 1a, 5 genotype 1b, 14 genotype 2, 18 genotype 3; 18 cirrhosis) who failed with previous DAA treatments containing sofosbuvir/velpatasvir for 12 weeks or a shorter duration (sofosbuvir/velpatasvir 27, sofosbuvir/velpatasvir and ribavirin 14, sofosbuvir/velpatasvir/voxilaprevir 28) were retreated with sofosbuvir/velpatasvir and ribavirin for 24 weeks. ${ }^{235}$ The overall SVR was $91 \%(63 / 69)$. The SVR by genotype was $97 \%$ (31/32) in genotype $1 \mathrm{a}, 100 \%(5 / 5)$ in genotype $1 \mathrm{~b}, 93 \%$ (13/14) in genotype 2 , and $78 \%(14 / 18)$ in genotype 3 . The SVR of the patients with cirrhosis was 78\% (14/18). The SVR in patients with NS5A RASs at baseline was 100\% (5/5) in genotype 1, 89\% (8/9) in genotype 2 , and $77 \%(10 / 13)$ in genotype 3 .

A total of 50 genotype $1 \mathrm{CHC}$ patients without cirrhosis (42 genotype 1a, 8 genotype 1b) who failed with previous DAA treatments (NS3 inhibitors 25, NS5A inhibitors 8, NS3 inhibitors and NS5A inhibitors 17) were retreated with glecaprevir/pibrentasvir with or without ribavirin for 12 weeks. The overall SVR was $92 \%$ (46/50). ${ }^{236}$ According to the dosage of glecaprevir/pibrentasvir, the SVR were $100 \%(6 / 6), 95 \%(21 / 22)$, and $86 \%$ (19/22) with $200 / 80 \mathrm{mg}, 300 / 120 \mathrm{mg}$ with ribavirin, and $300 / 120 \mathrm{mg}$, respectively. Recurrence occurred in 1 patient in each of the glecaprevir $(300 \mathrm{mg})$ /pibrentasvir (120 mg) with ribavirin and glecaprevir (300 $\mathrm{mg} /$ /pibrentasvir $(120 \mathrm{mg}$ ) groups. Two patients in the glecaprevir $(300 \mathrm{mg}) /$ pibrentasvir $(120 \mathrm{mg})$ group did not achieve SVR due to follow-up loss.

Ninety-one CHC patients (67 genotype 1a, 18 genotype 1b, 2 other genotype 1, 4 genotype 4, 27 cirrhosis) who failed with a previous DAA treatment (NS5A inhibitors 34, NS3 inhibitors 27, NS3, and NS5A inhibitors 30) were retreated with glecaprevir/pibrentasvir for 12 or 16 weeks. ${ }^{237}$ Overall, the SVR for the 12- and 16-week treatments were 89\% (39/44) and 91\% (43/47), respectively. According to the type of previous treatment failure, the SVR for the 12- and 16-week treatments were 100\% (14/14) and $100 \%(13 / 13)$ for previous NS3 inhibitor failures, 88\% (14/16) and 94\% (17/18) for previous NS5A inhibitor failures, and 79\% (11/14) and $81 \%(13 / 16)$ for previous NS3 and NS5A inhibitor failures, respectively. The SVR following 12 weeks and 16 weeks of glecaprevir/pibrentasvir was $100 \%(13 / 13)$ and $100 \%(13 / 13)$ in patients without baseline RASs, 100\% (2/2) and 100\% (4/4) in patients with NS3 RASs, 83\% (20/24) and 96\% (22/23) in patients with NS5A RASs, and $80 \%(4 / 5)$ and $25 \%(1 / 4)$ in patients with both NS3 and NS5A RASs, respectively. ${ }^{238}$ Therefore, based on the clinical study results to date, glecaprevir/pibrentasvir could have a limited efficacy in patients with both NS3 and NS5A RASs.

The SVR following 12 weeks of ledipasvir/sofosbuvir treatment was 100\% in 14 patients with genotype 1 CHC (8 genotype 1a, 6 genotype 1b) who failed with 24 weeks of sofosbuvir and ribavirin treatment. ${ }^{239}$ Fifty-one patients (30 genotype 1a, 20 genotype $1 \mathrm{~b}$, 1 genotype $3 a, 14$ cirrhosis) who failed with previous HCV therapy (sofosbuvir, peginterferon alpha, and ribavirin 25, sofosbuvir and ribavirin 20, peginterferon alpha and ribavirin without sofosbuvir 6) were retreated with ledipasvir/sofosbuvir with ribavirin for 12 weeks. The SVR was $98 \%(50 / 51){ }^{240}$

Fifty-two CHC patients (44 genotype 1, 2 genotype 2, 4 genotype 3, 3 genotype 4) who had previous treatment experience were retreated with daclatasvir and sofosbuvir for 12 weeks. The SVR was $98 \%$ (51/52), but the number of patients with genotypes 2 and 3 was very small. ${ }^{30}$ In a study of genotype 3 patients given daclatasvir and sofosbuvir for 12 weeks, patients with prior treatment experience (sofosbuvir and ribavirin or sofosbuvir and peginterferon alpha with ribavirin) did not show a satisfactory SVR $(71 \%, 5 / 7) .{ }^{83}$ Although the data are very limited, the combination of daclatasvir and sofosbuvir with ribavirin for 24 weeks could be considered in these patients.

In a study of genotype 3 CHC patients with cirrhosis, 53 patients who failed with previous treatment (including 2 on sofosbuvir and ribavirin combination therapy) were retreated with elbasvir/grazoprevir and sofosbuvir for 12 weeks, elbasvir/grazoprevir, sofosbuvir, and ribavirin for 12 weeks, or elbasvir/grazoprevir and sofosbuvir for 16 weeks. ${ }^{94}$ The SVR were 100\% (17/17), 94\% (17/18), and 94\% (17/18), respectively.

In two studies of genotype 1, 2, 4, 5, and 6 CHC patients without cirrhosis, a few of whom (1-6 patients in each genotype) had prior sofosbuvir-based treatment, glecaprevir/pibrentasvir for 8 and 12 weeks showed the SVR of $97-99 \%$ and $99-100 \%$, respectively. ${ }^{241,242} \mathrm{~A}$ total of 146 patients with genotype $1,2,4,5$, and 6 and cirrhosis (treatment experience 36, including 11 with 
prior sofosbuvir-based treatment) were retreated with glecaprevir/ pibrentasvir for 12 weeks, and the SVR was $99 \%{ }^{40}$ In 131 patients with genotype 3 (treatment-experience 91, including 42 with prior sofosbuvir-based treatment), 12 weeks of glecaprevir/ pibrentasvir treatment showed the SVR of 91\% (20/22) in patients without cirrhosis, and 16 weeks of glecaprevir/pibrentasvir treatment showed the SVR of 96\% (21/22) in patients without cirrhosis and $96 \%(45 / 47)$ in patients with cirrhosis. ${ }^{101}$

No studies have considered the efficacy of peginterferon in patients with DAA treatment failure. But peginterferon is expected to maintain its antiviral efficacy against several types of RASs, and therefore it could be a potential therapeutic option in patients who experience DAA treatment failure.

\section{[Recommendations] (Table 17)}

\section{General recommendation}

Patients with chronic hepatitis $C$ who have previously failed with DAA treatment could be retreated with currently available drugs, taking consideration into the previous treatment regimen, $\mathrm{HCV}$ genotype, presence of cirrhosis, and presence of RASs (B1).

Retreatment of DAA experienced patients (including NS5A inhibitors)

1. Treatment of patients with genotype 1 chronic hepatitis $C$ and compensated cirrhosis

(1) Sofosbuvir/velpatasvir/voxilaprevir should be administered for 12 weeks (A1).

(2) Sofosbuvir, elbasvir/grazoprevir, and ribavirin could be administered for 12 weeks (B1).

(3) Sofosbuvir and ombitasvir/paritaprevir/ritonavir plus dasabuvir could be administered for 12 weeks to patients with HCV genotype 1b (B1).

In patients with HCV genotype 1a, sofosbuvir and ombitasvir/ paritaprevir/ritonavir plus dasabuvir and ribavirin could be administered for 12 weeks to patients without liver cirrhosis and for 24 weeks to patients with liver cirrhosis (B1).

(4) Glecaprevir/pibrentasvir could be administered for 16 weeks (B1).

2. Treatment of patients with genotype $2,3,4,5$, or 6 chronic hepatitis $C$ and compensated cirrhosis

(1) Sofosbuvir/velpatasvir/voxilaprevir should be administered for 12 weeks (A1).

\section{Retreatment of DAA experienced patients (not including NS5A} inhibitors)

1. Treatment of patients with genotype 1 chronic hepatitis $C$ and compensated cirrhosis

(1) Sofosbuvir/velpatasvir/voxilaprevir should be administered for 12 weeks (A1).

(2) Glecaprevir/pibrentasvir could be administered for 12 weeks (B1).

(3) In patients with genotype 1b, sofosbuvir/velpatasvir could be administered for 12 weeks (B1).
2. Treatment of patients with genotype 2 chronic hepatitis $C$ and compensated cirrhosis

(1) Sofosbuvir/velpatasvir/voxilaprevir should be administered for 12 weeks (A1).

(2) Sofosbuvir/velpatasvir could be administered for 12 weeks (B1).

3. Treatment of patients with genotype 3 or 4 chronic hepatitis $C$ and compensated cirrhosis

(1) Sofosbuvir/velpatasvir/voxilaprevir should be administered for 12 weeks (A1).

Retreatment of sofosbuvir-based treatment-experienced patients (sofosbuvir, sofosbuvir with ribavirin, and sofosbuvir and peginterferon with ribavirin)

1. Treatment of patients with genotype 1 chronic hepatitis $C$ and compensated cirrhosis

(1) Sofosbuvir/velpatasvir/voxilaprevir should be administered for 12 weeks (A1).

(2) Glecaprevir/pibrentasvir could be administered for 12 weeks (B1).

(3) Ledipasvir/sofosbuvir and ribavirin could be administered for 12 weeks to patients without cirrhosis and for 24 weeks to patients with cirrhosis (B1).

2. Treatment of patients with genotype 2 chronic hepatitis $C$ and compensated cirrhosis

(1) Sofosbuvir/velpatasvir/voxilaprevir should be administered for 12 weeks (A1).

(2) Glecaprevir/pibrentasvir could be administered for 12 weeks (B1).

(3) Daclatasvir, sofosbuvir, and ribavirin could be administered for 24 weeks (C2).

3. Treatment of patients with genotype 3 chronic hepatitis $C$ and compensated cirrhosis

(1) Sofosbuvir/velpatasvir/voxilaprevir should be administered for 12 weeks (A1).

(2) Glecaprevir/pibrentasvir could be administered for 16 weeks (B1).

(3) Elbasvir/grazoprevir and sofosbuvir could be administered for 12 weeks (B1).

(4) Daclatasvir, sofosbuvir, and ribavirin could be administered for 24 weeks (C2).

4. Treatment of patients with genotype 4 chronic hepatitis $C$ and compensated cirrhosis

(1) Sofosbuvir/velpatasvir/voxilaprevir should be administered for 12 weeks (A1).

(2) Glecaprevir/pibrentasvir could be administered for 12 weeks (B1).

5. Treatment of patients with genotype 5 or 6 chronic hepatitis $C$ and compensated cirrhosis

(1) Glecaprevir/pibrentasvir could be administered for 12 weeks (B1).

\section{Conflicts of Interest}

Potential conflicts of interests are as follows:

Jong Eun Yeon: Received grants from Pharmaking, Bayer, BMS, Dong-A, Green Cross lab cell. 
The Korean Association for the Study of the Liver (KASL)

2017 KASL clinical practice guidelines: Management of chronic hepatitis C

Table 17. Retreatment of patients with direct-acting antiviral agent failure

\begin{tabular}{|c|c|c|c|c|c|c|c|c|}
\hline DAA failure & Genotype & SOF/VEL/VOX & $G / P^{*}$ & SOF+EBR/GZR & $\mathrm{SOF}+\mathrm{OPr}+\mathrm{D}$ & SOF/VEL & LDV/SOF & $\mathrm{DCV}+\mathrm{SOF}$ \\
\hline \multirow[t]{7}{*}{$\begin{array}{l}\text { NS5A inhibitor } \\
\text { experienced }\end{array}$} & 1a & $12 w k$ & $16 w k$ & $12 w k+R$ & $\begin{array}{l}12 w k+R(C H) \\
24 w k+R(L C)\end{array}$ & - & - & - \\
\hline & $1 b$ & 12 wk & $16 w k$ & $12 w k+R$ & 12 wk & - & - & - \\
\hline & 2 & $12 \mathrm{wk}$ & - & - & - & - & - & - \\
\hline & 3 & $12 w k$ & - & - & - & - & - & - \\
\hline & 4 & 12 wk & - & - & - & - & - & - \\
\hline & 5 & $12 \mathrm{wk}$ & - & - & - & - & - & - \\
\hline & 6 & $12 \mathrm{wk}$ & - & - & - & - & - & - \\
\hline \multirow{5}{*}{$\begin{array}{l}\text { Non-NS5A } \\
\text { inhibitor } \\
\text { experienced }\end{array}$} & $1 \mathrm{a}$ & $12 w k$ & $12 w k$ & - & - & - & - & - \\
\hline & $1 b$ & 12 wk & $12 w k$ & - & - & 12 wk & - & - \\
\hline & 2 & 12 wk & - & - & - & 12 wk & - & - \\
\hline & 3 & 12 wk & - & - & - & - & - & - \\
\hline & 4 & 12 wk & - & - & - & - & - & - \\
\hline \multirow{5}{*}{$\begin{array}{l}\text { SOF, SOF+RBV, } \\
\text { SOF+PR } \\
\text { experienced }\end{array}$} & 1 & $12 \mathrm{wk}$ & $12 \mathrm{wk}$ & - & - & - & $\begin{array}{l}12 w k+R(C H) \\
24 w k+R(L C)\end{array}$ & - \\
\hline & 2 & $12 w k$ & $12 w k$ & - & - & - & - & $24 w k+R$ \\
\hline & 3 & 12 wk & 16 wk & 12 wk & - & - & - & $24 w k+R$ \\
\hline & 4 & $12 \mathrm{wk}$ & $12 w k$ & - & - & - & - & - \\
\hline & 5,6 & & $12 w k$ & - & - & - & - & - \\
\hline
\end{tabular}

DAA, direct-acting antivirals; SOF, sofosbuvir; VEL, velpatasvir; VOX, voxilaprevir; G, glecaprevir; P, pibrentasvir; EBR, elbasvir; GZR, grazoprevir; OPr, ombitasvir/ paritaprevir/ritonavir; D, dasabuvir; LDV, ledipasvir; DCV, daclatasvir; wk, weeks; R, weight-based ribavirin; CH, chronic hepatitis; LC, liver cirrhosis; RBV, ribavirin; PR, peginterferon alpha+ribavirin.

*Indicated in patients who have been treated with regimens containing NS5A or NS3/4A inhibitors, not both.

In Hee Kim: Received honoraria from MSD, Abbvie, Yuhan. Received grants from BMS, Dong-A.

Geum-Youn Gwak: Received honoraria from BMS, Samjin, Dong-A. Received grants from Gilead, BMS, KOWA, SillaJen, Biocompatibles, GSK, Altimmune, Abbvie.

Jung II Lee: Received honoraria from BMS, Gilead. Received grants from MSD, BMS, Gilead, Bayer, KOWA, Eisai, Novotech, EPS, Ildong, Medigen Biotechnology.

Kyung-Ah Kim: Received grants from MSD, BMS, Gilead.

Ji Hoon Kim: Received honoraria from Gilead, BMS, MSD, Abbvie, Chongkundang, Yuhan, Dong-A, Menarini, Daewoong, Teva. Consulted for Gilead, Abbvie, Chongkundang, Dong-A. Received grants from Gilead, BMS, MSD, Abbvie, Chongkundang, Yuhan, Dong-A, Daewoong, KOWA.

Kang Mo Kim: Received honoraria from Chongkundang, DongA, Abbvie, Bayer. Consulted for Bayer, MSD, Chongkundang, GSK. Received grants from Bayer, Dong-A, Yuhan, JW Creagene, MSD.

Jeong Won Jang: Received honoraria from Celltrion, MSD, Abbvie, BMS, Bayer. Received grants from GSK, Altimmune, Sillagen,
Spring Bank, Yuhan, Chongkundang, Dong-A.

Do Young Kim: Received honoraria from Abbvie, BMS, Gilead, MSD, Bayer, Eisai, Sirtex, BTG, Yuhan. Consulted for Bayer, SIRTEX, Ono. Received grants from Gilead, Bayer.

Ki Tae Yoon: Received grants from Abbvie, Bayer, BMS, Gilead.

\section{REFERENCES}

1. Guyatt GH, Oxman AD, Vist GE, Kunz R, Falck-Ytter $Y$, AlonsoCoello $P$, et al. GRADE: an emerging consensus on rating quality of evidence and strength of recommendations. BMJ 2008;336:924926.

2. Falade-Nwulia O, Suarez-Cuervo C, Nelson DR, Fried MW, Segal $J B$, Sulkowski MS. Oral direct-acting agent therapy for hepatitis $C$ virus infection: a systematic review. Ann Intern Med 2017;166:637648.

3. Sciences G. Sovaldi(R) (sofosbuvir), prescribing information. Gilead web site, <http://www.gilead.com/ /media/Files/pdfs/medicines/ liver-disease/sovaldi/sovaldi_pi.pdf>. Accessed Sep 2017. 
4. Squibb B-M. Daklinza(R) (daclatasvir), prescribing information. Bristol-Myers Squibb web site, <http://packageinserts.bms.com/pi/ pi_daklinza.pdf>. Accessed Sep 2017.

5. AbbVie. Viekira(R) (dasabuvir, ombitasvir, paritaprevir, and ritonavir), prescribing information. AbbVie web site, <http://www.rxabbvie.com/pdf/viekirapak_pi.pdf>. Accessed Sep 2017.

6. MSD. Zepatier(R) (elbasvir and grazoprevir), prescribing information. MSD web site, <https://www.merck.com/product/usa/pi_ circulars/z/zepatier/zepatier_pi.pdf>. Accessed Sep 2017.

7. AbbVie. Mavyret(R) (glecaprevir and pibrentasvir), prescribing information. AbbVie web site, <http://www.rxabbvie.com/pdf/mavyret_pi.pdf>. Accessed Sep 2017.

8. Sciences G. Epclusa(R) (sofosbuvir and velpatasvir), prescribing information. Gilead web site, <http://www.gilead.com/ /media/ Files/pdfs/medicines/liver-disease/epclusa/epclusa_pi.pdf>. Accessed Sep 2017.

9. Pawlotsky JM. Hepatitis C virus resistance to direct-acting antiviral drugs in interferon-free regimens. Gastroenterology 2016;151:7086.

10. Sarrazin C. The importance of resistance to direct antiviral drugs in HCV infection in clinical practice. J Hepatol 2016;64:486-504.

11. Gane EJ, Abergel A, Metivier S, Nahass R, Ryan M, Stedman CA, et al. The emergence of NS5B resistant associated variant S282T after sofosbuvir-based treatment [Abstract]. Hepatology 2015;62:322A.

12. Manns M, Pol S, Jacobson IM, Marcellin P, Gordon SC, Peng CY. All-oral daclatasvir plus asunaprevir for hepatitis $C$ virus genotype 1b: a multinational, phase 3, multicohort study. Lancet 2014;384:1597-1605.

13. Zeuzem S, Ghalib R, Reddy KR, Pockros PJ, Ari ZB, Zhao Y, et al. Grazoprevir-elbasvir combination therapy for treatment-naive cirrhotic and noncirrhotic patients with chronic hepatitis $C$ virus genotype 1, 4, or 6 infection: a randomized trial. Ann Intern Med 2015;163:1-13.

14. Afdhal N, Zeuzem S, Kwo P, Chojkier M, Gitlin N, Puoti M, et al. Ledipasvir and sofosbuvir for untreated HCV genotype 1 infection. N Engl J Med 2014;370:1889-1898.

15. Kowdley KV, Gordon SC, Reddy KR, Rossaro L, Bernstein DE, Lawitz $E$, et al. Ledipasvir and sofosbuvir for 8 or 12 weeks for chronic HCV without cirrhosis. N Engl J Med 2014;370:1879-1888.

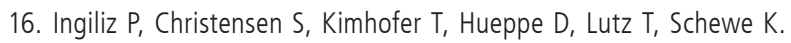
Sofosbuvir and ledipasvir for 8 Weeks for the treatment of chronic hepatitis $\mathrm{C}$ virus (HCV) infection in HCV-monoinfected and HIVHCV-coinfected individuals: results from the German hepatitis C cohort (GECCO-01). Clin Infect Dis 2016;63:1320-1324.

17. Zeng QL, Xu GH, Zhang JY, Li W, Zhang DW, Li ZQ, et al. Generic ledipasvir-sofosbuvir for patients with chronic hepatitis C: a reallife observational study. J Hepatol 2017;66:1123-1129.

18. Backus LI, Belperio PS, Shahoumian TA, Loomis TP, Mole LA. Real- world effectiveness of ledipasvir/sofosbuvir in 4,365 treatmentnaive, genotype 1 hepatitis C-infected patients. Hepatology 2016;64:405-414.

19. Kowdley KV, Sundaram V, Jeon CY, Qureshi K, Latt NL, Sahota A, et al. Eight weeks of ledipasvir/sofosbuvir is effective for selected patients with genotype 1 hepatitis C virus infection. Hepatology 2017;65:1094-1103.

20. Zeuzem S, Mizokami M, Pianko S, Mangia A, Han KH, Martin R, et al. NS5A resistance-associated substitutions in patients with genotype 1 hepatitis $C$ virus: prevalence and effect on treatment outcome. J Hepatol 2017;66:910-918.

21. Ogawa E, Furusyo N, Nomura H, Dohmen K, Higashi N, Takahashi K, et al. NS5A resistance-associated variants undermine the effectiveness of ledipasvir and sofosbuvir for cirrhotic patients infected with HCV genotype 1b. J Gastroenterol 2017; 52:845-854.

22. Sperl J, Horvath G, Halota W, Ruiz-Tapiador JA, Streinu-Cercel A, Jancoriene $L$, et al. Efficacy and safety of elbasvir/grazoprevir and sofosbuvir/pegylated interferon/ribavirin: a phase III randomized controlled trial. J Hepatol 2016;65:1112-1119.

23. Kumada H, Suzuki Y, Karino Y, Chayama K, Kawada N, Okanoue T, et al. The combination of elbasvir and grazoprevir for the treatment of chronic HCV infection in Japanese patients: a randomized phase II/III study. J Gastroenterol 2017;52:520-533.

24. Komatsu TE, Boyd S, Sherwat A, Tracy L, Naeger LK, O'Rear JJ, et al. Regulatory analysis of effects of hepatitis C virus NS5A polymorphisms on efficacy of elbasvir and grazoprevir. Gastroenterology 2017;152:586-597.

25. Ferenci P, Bernstein D, Lalezari J, Cohen D, Luo Y, Cooper C, et al. ABT-450/r-ombitasvir and dasabuvir with or without ribavirin for HCV. N Engl J Med 2014;370:1983-1992.

26. Dore GJ, Conway B, Luo Y, Janczewska E, Knysz B, Liu Y, et al. Efficacy and safety of ombitasvir/paritaprevir/r and dasabuvir compared to IFN-containing regimens in genotype $1 \mathrm{HCV}$ patients: the MALACHITE-I/II trials. J Hepatol 2016;64:19-28.

27. Poordad F, Hezode C, Trinh R, Kowdley KV, Zeuzem S, Agarwal K, et al. ABT-450/r-ombitasvir and dasabuvir with ribavirin for hepatitis C with cirrhosis. N Engl J Med 2014;370:1973-1982.

28. Feld JJ, Moreno C, Trinh R, Tam E, Bourgeois S, Horsmans Y, et al. Sustained virologic response of $100 \%$ in HCV genotype $1 \mathrm{~b}$ patients with cirrhosis receiving ombitasvir/paritaprevir/r and dasabuvir for 12weeks. J Hepatol 2016;64:301-307.

29. Luetkemeyer AF, McDonald C, Ramgopal M, Noviello S, Bhore R, Ackerman P. 12 weeks of daclatasvir in combination with sofosbuvir for HIV-HCV coinfection (ALLY-2 Study): efficacy and safety by HIV combination antiretroviral regimens. Clin Infect Dis 2016:62:1489-1496.

30. Wyles DL, Ruane PJ, Sulkowski MS, Dieterich D, Luetkemeyer A, Morgan TR, et al. Daclatasvir plus sofosbuvir for HCV in patients 
The Korean Association for the Study of the Liver (KASL) 2017 KASL clinical practice guidelines: Management of chronic hepatitis C

coinfected with HIV-1. N Engl J Med 2015;373:714-725.

31. Sulkowski MS, Gardiner DF, Rodriguez-Torres M, Reddy KR, Hassanein $T$, Jacobson I, et al. Daclatasvir plus sofosbuvir for previously treated or untreated chronic HCV infection. N Engl J Med 2014;370:211-221.

32. Pol S, Bourliere M, Lucier S, Hezode C, Dorival C, Larrey D, et al. Safety and efficacy of daclatasvir-sofosbuvir in HCV genotype 1-mono-infected patients. J Hepatol 2017;66:39-47.

33. Poordad F, Schiff ER, Vierling JM, Landis C, Fontana RJ, Yang R, et al. Daclatasvir with sofosbuvir and ribavirin for hepatitis $C$ virus infection with advanced cirrhosis or post-liver transplantation recurrence. Hepatology 2016;63:1493-1505.

34. McPhee F, Suzuki Y, Toyota J, Karino Y, Chayama K, Kawakami Y, et al. High sustained virologic response to daclatasvir plus asunaprevir in elderly and cirrhotic patients with hepatitis C virus genotype 1b without baseline NS5A polymorphisms. Adv Ther 2015;32:637649.

35. Kao JH, Lee YJ, Heo J, Ahn SH, Lim YS, Peng CY, et al. All-oral daclatasvir plus asunaprevir for chronic hepatitis $\mathrm{C}$ virus (HCV) genotype $1 \mathrm{~b}$ infection: a sub-analysis in Asian patients from the HALLMARK DUAL study. Liver Int 2016;36:1433-1441.

36. Kwo PY, Poordad F, Asatryan A, Wang S, Wyles DL, Hassanein $T$, et al. Glecaprevir and pibrentasvir yield high response rates in patients with HCV genotype 1-6 without cirrhosis. J Hepatol 2017;67:263-271.

37. Zeuzem S, Feld JJ, Wang S, Bourliere M, Wedemeyer H, Gane EJ, et al. ENDURANCE-1: efficacy and safety of 8-versus 12-week treatment with ABT-493/ABT-530 in patients with chronic HCV genotype 1 infection [Abstract]. Hepatology 2016;64:132A-133A.

38. Puoti M, Foster G, Wang S, Mutimer DJ, Gane E, Moreno C, et al. High SVR rates with eight and twelve weeks of pangenotypic glecaprevir/pibrentasvir: integrated efficacy and safety analysis of genotype 1-6 patients without cirrhosis [Abstract]. J Hepatol 2017;66:S721.

39. Gane E, Poordad F, Wang S, Asatryan A, Kwo PY, Lalezari J, et al. High efficacy of ABT-493 and ABT-530 treatment in patients with HCV genotype 1 or 3 infection and compensated cirrhosis. Gastroenterology 2016;151:651-659.

40. Forns X, Lee SS, Valdes J, Lens S, Ghalib R, Aguilar H, et al. Glecaprevir plus pibrentasvir for chronic hepatitis $C$ virus genotype 1,2 , 4,5 , or 6 infection in adults with compensated cirrhosis (EXPEDITION-1): a single-arm, open-label, multicentre phase 3 trial. Lancet Infect Dis 2017;17:1062-1068.

41. Chayama K, Suzuki F, Karino Y, Kawakami Y, Sato K, Atarashi T, et al. Efficacy and safety of glecaprevir/pibrentasvir in Japanese patients with chronic genotype 1 hepatitis C virus infection with and without cirrhosis. J Gastroenterol 2018;53:557-565.

42. Feld JJ, Jacobson IM, Hézode C, Asselah T, Ruane PJ, Gruener N, et al. Sofosbuvir and velpatasvir for HCV genotype 1, 2, 4, 5, and 6 infection. N Engl J Med 2015;373:2599-2607.

43. Jacobson IM, Lawitz E, Gane EJ, Willems BE, Ruane PJ, Nahass RG, et al. Efficacy of 8 weeks of sofosbuvir, velpatasvir, and voxilaprevir in patients with chronic HCV infection: 2 phase 3 randomized trials. Gastroenterology 2017;153:113-122.

44. Afdhal N, Reddy KR, Nelson DR, Lawitz E, Gordon SC, Schiff E, et al. Ledipasvir and sofosbuvir for previously treated HCV genotype 1 infection. N Engl J Med 2014;370:1483-1493.

45. Bourlière $M$, Bronowicki JP, de Ledinghen $V$, Hézode $C$, Zoulim $F$, Mathurin $\mathrm{P}$, et al. Ledipasvir-sofosbuvir with or without ribavirin to treat patients with HCV genotype 1 infection and cirrhosis nonresponsive to previous protease-inhibitor therapy: a randomised, double-blind, phase 2 trial (SIRIUS). Lancet Infect Dis 2015;15:397404.

46. Mizokami M, Yokosuka O, Takehara T, Sakamoto N, Korenaga M, Mochizuki $\mathrm{H}$, et al. Ledipasvir and sofosbuvir fixed-dose combination with and without ribavirin for 12 weeks in treatment-naive and previously treated Japanese patients with genotype 1 hepatitis C: an open-label, randomised, phase 3 trial. Lancet Infect Dis 2015;15:645-653.

47. Lim YS, Ahn SH, Lee KS, Paik SW, Lee YJ, Jeong SH, et al. A phase IIlb study of ledipasvir/sofosbuvir fixed-dose combination tablet in treatment-naive and treatment-experienced Korean patients chronically infected with genotype 1 hepatitis C virus. Hepatol Int 2016;10:947-955.

48. Sarrazin C, Dvory-Sobol H, Svarovskaia ES, Doehle BP, Pang PS, Chuang SM, et al. Prevalence of resistance-associated substitutions in HCV NS5A, NS5B, or NS3 and outcomes of treatment with ledipasvir and sofosbuvir. Gastroenterology 2016;151:501-512.e1.

49. Kwo P, Gane EJ, Peng CY, Pearlman B, Vierling JM, Serfaty L, et al. Effectiveness of elbasvir and grazoprevir combination, with or without ribavirin, for treatment-experienced patients with chronic hepatitis C infection. Gastroenterology 2017;152:164-175.e4.

50. Zeuzem S, Jacobson IM, Baykal T, Marinho RT, Poordad F, Bourlière $M$, et al. Retreatment of HCV with ABT-450/r-ombitasvir and dasabuvir with ribavirin. N Engl J Med 2014;370:1604-1614.

51. Andreone P, Colombo MG, Enejosa JV, Koksal I, Ferenci P, Maieron A, et al. ABT-450, ritonavir, ombitasvir, and dasabuvir achieves $97 \%$ and $100 \%$ sustained virologic response with or without ribavirin in treatment-experienced patients with HCV genotype $1 \mathrm{~b}$ infection. Gastroenterology 2014;147:359-365.

52. Kumada H, Suzuki Y, Ikeda K, Toyota J, Karino Y, Chayama K, et al. Daclatasvir plus asunaprevir for chronic HCV genotype $1 \mathrm{~b}$ infection. Hepatology 2014;59:2083-2091.

53. Wang HL, Lu X, Yang X, Xu N. Effectiveness and safety of daclatasvir plus asunaprevir for hepatitis $C$ virus genotype $1 \mathrm{~b}$ : Systematic review and meta-analysis. J Gastroenterol Hepatol 2017;32:45-52. 
54. Shin SK, Kwon OS, Yoon CH, Jin YJ, Lee JW, Lee SH, et al. Efficacy of daclatasvir and asunaprevir treatment in genotype $1 \mathrm{~b} \mathrm{HCV}$ infected patients: a real life and multicenter study [Abstract]. Clin Mol Hepatol 2017;23(Suppl 2):59A.

55. Lee HW, Jung HK, Kim BK, Kim SU, Kim DY, Ahn SH, et al. The real-life data of daclatasvir and asunaprevir treatment in Korean patients with hepatitis C genotype $1 \mathrm{~b}$ infection [Abstract]. Clin Mol Hepatol 2017;23(Suppl 2):63A.

56. Pianko S, Flamm SL, Shiffman ML, Kumar S, Strasser SI, Dore GJ, et al. Sofosbuvir plus velpatasvir combination therapy for treatmentexperienced patients with genotype 1 or 3 hepatitis C virus infection: a randomized trial. Ann Intern Med 2015;163:809-817.

57. Alqahtani SA, Afdhal N, Zeuzem S, Gordon SC, Mangia A, Kwo P, et al. Safety and tolerability of ledipasvir/sofosbuvir with and without ribavirin in patients with chronic hepatitis C virus genotype 1 infection: Analysis of phase III ION trials. Hepatology 2015;62:2530.

58. Lawitz E, Gane E, Pearlman B, Tam E, Ghesquiere W, Guyader D, et al. Efficacy and safety of 12 weeks versus 18 weeks of treatment with grazoprevir (MK-5172) and elbasvir (MK-8742) with or without ribavirin for hepatitis C virus genotype 1 infection in previously untreated patients with cirrhosis and patients with previous null response with or without cirrhosis (C-WORTHY): a randomised, open-label phase 2 trial. Lancet 2015;385:1075-1086.

59. Jacobson IM, Asante-Appiah E, Wong P, Black T, Howe AY, Wahl J, et al. Prevalence and impact of baseline NSA resistance associated variants (RAVs) on the efficacy of elbasvir/grazoprevir (EBR/GZR) against GT1a infection [Abstract]. Hepatology 2015;62:1393A1394A.

60. Thompson AJ, Zeuzum S, Rockstroh JK, Kwo PY, Roth D, Lawitz E, et al. The combination of grazoprevir and elbasvir \pm RbV Is highly effective for the treatment of GT1a-infected patients [Abstract]. Hepatology 2015;62:556A-557A.

61. Feld JJ, Kowdley KV, Coakley E, Sigal S, Nelson DR, Crawford D, et al. Treatment of HCV with ABT-450/r-ombitasvir and dasabuvir with ribavirin. N Engl J Med 2014;370:1594-1603.

62. Jacobson IM, Gordon SC, Kowdley KV, Yoshida EM, RodriguezTorres M, Sulkowski MS, et al. Sofosbuvir for hepatitis $C$ genotype 2 or 3 in patients without treatment options. N Engl J Med 2013;368:1867-1877.

63. Ahn SH, Lim YS, Lee KS, Paik SW, Lee YJ, Jeong SH, et al. A phase $3 \mathrm{~b}$ study of sofosbuvir plus ribavirin in treatment-naive and treatment-experienced Korean patients chronically infected with genotype 2 hepatitis C virus. J Viral Hepat 2016;23:358-365.

64. Omata M, Nishiguchi S, Ueno Y, Mochizuki H, Izumi N, Ikeda F, et al. Sofosbuvir plus ribavirin in Japanese patients with chronic genotype 2 HCV infection: an open-label, phase 3 trial. J Viral Hepat 2014;21:762-768.
65. Zeuzem S, Dusheiko GM, Salupere R, Mangia A, Flisiak R, Hyland $\mathrm{RH}$, et al. Sofosbuvir and ribavirin in HCV genotypes 2 and 3. N Engl J Med 2014;370:1993-2001.

66. Lee SW, Lee HL, Han NI, Kim HY, Kim CW, You CR, et al. Real-life experience of sofosbuvir and ribavirin for genotype $2 \mathrm{HCV}$ infected Korean patients: a multicenter cohort study [Abstract]. Clin Mol Hepatol 2017;23(Suppl 2):62A-63A

67. Welzel TM, Nelson DR, Morelli G, Di Bisceglie A, Reddy RK, Kuo A, et al. Effectiveness and safety of sofosbuvir plus ribavirin for the treatment of HCV genotype 2 infection: results of the real-world, clinical practice HCV-TARGET study. Gut 2017;66:1844-1852.

68. Mangia A, Susser S, Piazzolla V, Agostinacchio E, De Stefano G, Palmieri $V$, et al. Sofosbuvir and ribavirin for genotype $2 \mathrm{HCV}$ infected patients with cirrhosis: a real life experience. J Hepatol 2017;66:711-717.

69. Ippolito AM, Milella M, Messina V, Conti F, Cozzolongo R, Morisco $F$, et al. HCV clearance after direct-acting antivirals in patients with cirrhosis by stages of liver impairment: the ITAL-C network study. Dig Liver Dis 2017;49:1022-1028.

70. Foster GR, Afdhal N, Roberts SK, Bräu N, Gane EJ, Pianko S, et al. Sofosbuvir and velpatasvir for HCV genotype 2 and 3 infection. N Engl J Med 2015;373:2608-2617.

71. Agarwal K, Patel K, Samuel D, Bourliere M, Younes Z, Morgan T, et al. SOF/VEL for 12 weeks results in high SVR12 rates in subjects with negative predictors of response to treatment: an integrated analysis of efficacy from the Astral-1, Astral-2 and Astral-3 Studies [Abstract]. J Hepatol 2016;64:S787-S788.

72. Lee S, Kim IH, Kim SH, Kim SW, Lee SO, Lee ST, et al. Efficacy and tolerability of pegylated interferon-alpha2a plus ribavirin versus pegylated interferon-alpha2b plus ribavirin in treatment-naive chronic hepatitis C patients. Intervirology 2010;53:146-153.

73. Park SH, Park CK, Lee JW, Kim YS, Jeong SH, Kim YS, et al. Efficacy and tolerability of peginterferon alpha plus ribavirin in the routine daily treatment of chronic hepatitis C patients in Korea: a multicenter, retrospective observational study. Gut Liver 2012;6:98-106.

74. Ferenci P, Brunner H, Laferl H, Scherzer TM, Maieron A, Strasser $M$, et al. A randomized, prospective trial of ribavirin $400 \mathrm{mg} /$ day versus $800 \mathrm{mg} /$ day in combination with peginterferon alfa-2a in hepatitis C virus genotypes 2 and 3. Hepatology 2008:47:18161823.

75. Fried MW, Shiffman ML, Reddy KR, Smith C, Marinos G, Gonçales $\mathrm{FL} \mathrm{Jr}$, et al. Peginterferon alfa-2a plus ribavirin for chronic hepatitis C virus infection. N Engl J Med 2002;347:975-982.

76. Hadziyannis SJ, Sette H Jr, Morgan TR, Balan V, Diago M, Marcellin $P$, et al. Peginterferon-alpha2a and ribavirin combination therapy in chronic hepatitis C: a randomized study of treatment duration and ribavirin dose. Ann Intern Med 2004;140:346-355.

77. Jacobson IM, Brown RS Jr, Freilich B, Afdhal N, Kwo PY, Santoro J, 
The Korean Association for the Study of the Liver (KASL) 2017 KASL clinical practice guidelines: Management of chronic hepatitis C

et al. Peginterferon alfa-2b and weight-based or flat-dose ribavirin in chronic hepatitis C patients: a randomized trial. Hepatology 2007:46:971-981.

78. Manns MP, McHutchison JG, Gordon SC, Rustgi VK, Shiffman M, Reindollar $\mathrm{R}$, et al. Peginterferon alfa-2b plus ribavirin compared with interferon alfa-2b plus ribavirin for initial treatment of chronic hepatitis C: a randomised trial. Lancet 2001;358:958-965.

79. Zeuzem S, Hultcrantz R, Bourliere M, Goeser T, Marcellin P, Sanchez-Tapias J, et al. Peginterferon alfa-2b plus ribavirin for treatment of chronic hepatitis $\mathrm{C}$ in previously untreated patients infected with HCV genotypes 2 or 3. J Hepatol 2004;40:993-999.

80. Foster GR, Pianko S, Brown A, Forton D, Nahass RG, George J, et al. Efficacy of sofosbuvir plus ribavirin with or without peginterferon-alfa in patients with hepatitis C virus genotype 3 infection and treatment-experienced patients with cirrhosis and hepatitis $C$ virus genotype 2 infection. Gastroenterology 2015;149:1462-1470.

81. Gane EJ, Hyland RH, An D, Svarovskaia E, Pang PS, Brainard D, et al. Efficacy of ledipasvir and sofosbuvir, with or without ribavirin, for 12 weeks in patients with HCV genotype 3 or 6 infection. Gastroenterology 2015;149:1454-1461.

82. Gao M, Nettles RE, Belema M, Snyder LB, Nguyen VN, Fridell RA, et al. Chemical genetics strategy identifies an HCV NS5A inhibitor with a potent clinical effect. Nature 2010;465:96-100.

83. Nelson DR, Cooper JN, Lalezari JP, Lawitz E, Pockros PJ, Gitlin N, et al. All-oral 12-week treatment with daclatasvir plus sofosbuvir in patients with hepatitis C virus genotype 3 infection: ALLY-3 phase III study. Hepatology 2015;61:1127-1135.

84. Leroy V, Angus P, Bronowicki JP, Dore GJ, Hezode C, Pianko S, et al. Daclatasvir, sofosbuvir, and ribavirin for hepatitis $C$ virus genotype 3 and advanced liver disease: a randomized phase III study (ALLY-3+). Hepatology 2016;63:1430-1441.

85. Alonso S, Riveiro-Barciela M, Fernandez I, Rincón $D$, Real Y, Llerena $S$, et al. Effectiveness and safety of sofosbuvir-based regimens plus an NS5A inhibitor for patients with HCV genotype 3 infection and cirrhosis. Results of a multicenter real-life cohort. J Viral Hepat 2017;24:304-311.

86. Welzel TM, Petersen J, Herzer K, Ferenci P, Gschwantler M, Wedemeyer $\mathrm{H}$, et al. Daclatasvir plus sofosbuvir, with or without ribavirin, achieved high sustained virological response rates in patients with HCV infection and advanced liver disease in a real-world cohort. Gut 2016;65:1861-1870.

87. Foster GR, Gane E, Asatryan A, Asselah T, Ruane PJ, Pol S, et al. ENDURANCE-3: safety and efficacy of glecaprevir/pibrentasvir compared to sofosbuvir plus daclatasvir in treatment-nave HCV genotype 3-infected patients without cirrhosis [Abstract]. J Hepatol 2017;66:S33.

88. Gane EJ, Stedman CA, Hyland RH, Ding X, Svarovskaia E, Symonds WT, et al. Nucleotide polymerase inhibitor sofosbuvir plus ribavirin for hepatitis C. N Engl J Med 2013;368:34-44.

89. Lawitz E, Lalezari JP, Hassanein T, Kowdley KV, Poordad FF, Sheikh $A M$, et al. Sofosbuvir in combination with peginterferon alfa-2a and ribavirin for non-cirrhotic, treatment-naive patients with genotypes 1, 2, and 3 hepatitis C infection: a randomised, double-blind, phase 2 trial. Lancet Infect Dis 2013;13:401-408.

90. Dalgard O, Bjøro K, Ring-Larsen H, Bjornsson E, Holberg-Petersen $M$, Skovlund $E$, et al. Pegylated interferon alfa and ribavirin for 14 versus 24 weeks in patients with hepatitis C virus genotype 2 or 3 and rapid virological response. Hepatology 2008;47:35-42.

91. Mangia A, Santoro R, Minerva N, Ricci GL, Carretta V, Persico M, et al. Peginterferon alfa-2b and ribavirin for 12 vs. 24 weeks in HCV genotype 2 or 3. N Engl J Med 2005;352:2609-2617.

92. Shiffman ML, Suter F, Bacon BR, Nelson D, Harley $H$, Solá R, et al. Peginterferon alfa-2a and ribavirin for 16 or 24 weeks in HCV genotype 2 or 3. N Engl J Med 2007;357:124-134.

93. Hezode C, Lebray P, De Ledinghen V, Zoulim F, Di Martino V, Boyer $N$, et al. Daclatasvir plus sofosbuvir, with or without ribavirin, for hepatitis C virus genotype 3 in a French early access programme. Liver Int 2017;37:1314-1324.

94. Foster GR, Agarwal K, Cramp ME, Moreea S, Barclay S, Collier J, et al. Elbasvir/grazoprevir plus sofosbuvir in treatment-naive and treatment-experienced cirrhotic patients with hepatitis $C$ virus genotype 3 infection treated for 8, 12, or 16 weeks: final results of the C-ISLE study [Abstract]. J Hepatol 2017;66:S503-S504.

95. Wyles DL, Poordad F, Wang S, Alric L, Felizarta F, Kwo PY, et al. SURVEYOR-II, Part 3: efficacy and safety of glecaprevir/pibrentasvir (ABT-493/ABT-530) in patients with hepatitis C virus genotype 3 infection with prior treatment experience and/or cirrhosis [Abstract]. Hepatology 2016;64(Suppl):62A-63A.

96. Kohli A, Kapoor R, Sims Z, Nelson A, Sidharthan S, Lam B, et al. Ledipasvir and sofosbuvir for hepatitis $C$ genotype 4: a proof-ofconcept, single-centre, open-label phase 2a cohort study. Lancet Infect Dis 2015;15:1049-1054.

97. Abergel A, Metivier S, Samuel D, Jiang D, Kersey K, Pang PS, et al. Ledipasvir plus sofosbuvir for 12 weeks in patients with hepatitis $C$ genotype 4 infection. Hepatology 2016;64:1049-1056.

98. Asselah T, Reesink HW, Gerstoft J, de Ledinghen V, Pockros PJ, Robertson $M$, et al. High efficacy of grazoprevir and elbasvir with or without ribavirin in 103 treatment-naïve and experienced patients with HCV genotype 4 infection: a pooled analysis [Abstract]. Hepatology 2015;62:340A.

99. Rockstroh JK, Nelson M, Katlama C, Lalezari J, Mallolas J, Bloch $M$, et al. Efficacy and safety of grazoprevir (MK-5172) and elbasvir (MK-8742) in patients with hepatitis C virus and HIV co-infection (C-EDGE CO-INFECTION): a non-randomised, open-label trial. Lancet HIV 2015;2:e319-e327.

100. Hézode C, Asselah T, Reddy KR, Hassanein T, Berenguer M, Fleisch- 
er-Stepniewska K, et al. Ombitasvir plus paritaprevir plus ritonavir with or without ribavirin in treatment-naive and treatment-experienced patients with genotype 4 chronic hepatitis $C$ virus infection (PEARL-I): a randomised, open-label trial. Lancet 2015;385:25022509.

101. Asselah T, Hezode C, Zadeikis N, Elkhashab M, Colombo M, Marinho RT, et al. ENDURANCE-4: efficacy and safety of ABT-493/ ABT-530 treatment in patients with chronic HCV genotype 4, 5, or 6 infection [Abstract]. Hepatology 2016;63(Suppl):63A.

102. Fontaine $H$, Hezode C, Zoulim F, Samuel D, Bourliere M, Haour G, et al. LP28: Efficacy of the oral sofosbuvir-based combinations in HCV genotype 4-mono-infected patients from the french observational cohort anrs $\mathrm{CO} 22$ hepather [Abstract]. The International Liver Congress(TM) 2015-50th Annual meeting of the European Association for the Study of the Liver 2015;62:S278.

103. Hézode C, Abergel A, Chas J, Conti F, Cotte L, Tateo M, et al. Sustained virologic response to daclatasvir and sofosbuvir, with or without ribavirin, among patients in the French daclatasvir ATU programme infected with HCV genotypes 4, 5 and 6 [Abstract]. J Hepatol 2016;64:S755.

104. Abergel A, Asselah T, Metivier S, Kersey K, Jiang D, Mo H, et al. Ledipasvir-sofosbuvir in patients with hepatitis $C$ virus genotype 5 infection: an open-label, multicentre, single-arm, phase 2 study. Lancet Infect Dis 2016;16:459-464.

105. Wyles D, Bräu N, Kottilil S, Daar ES, Ruane P, Workowski K, et al. Sofosbuvir and velpatasvir for the treatment of hepatitis $C$ virus in patients coinfected with human immunodeficiency virus type 1: an open-label, phase 3 study. Clin Infect Dis 2017;65:6-12.

106. Nguyen MH, Keeffe EB. Chronic hepatitis C: genotypes 4 to 9. Clin Liver Dis 2005;9:411-26, vi.

107. Wong RJ, Nguyen MT, Trinh HN, Huynh A, Ly MT, Nguyen HA, et al. Community-based real-world treatment outcomes of sofosbuvir/ ledipasvir in Asians with chronic hepatitis C virus genotype 6 in the United States. J Viral Hepat 2017;24:17-21.

108. Nguyen NH, VuTien P, Garcia RT, Trinh H, Nguyen H, Nguyen K, et al. Response to pegylated interferon and ribavirin in Asian American patients with chronic hepatitis C genotypes 1 vs $2 / 3$ vs 6 . J Viral Hepat 2010;17:691-697.

109. Tsang OT, Zee JS, Chan JM, Li RS, Kan YM, Li FT, et al. Chronic hepatitis $C$ genotype 6 responds better to pegylated interferon and ribavirin combination therapy than genotype 1. J Gastroenterol Hepatol 2010;25:766-771.

110. Yuen MF, Lai $\mathrm{CL}$. Response to combined interferon and ribavirin is better in patients infected with hepatitis C virus genotype 6 than genotype 1 in Hong Kong. Intervirology 2006;49:96-98.

111. Bunchorntavakul C, Reddy KR. Treat chronic hepatitis C virus infection in decompensated cirrhosis - pre- or post-liver transplantation? the ironic conundrum in the era of effective and well-tolerated therapy. J Viral Hepat 2016;23:408-418.

112. Charlton M, Everson GT, Flamm SL, Kumar P, Landis C, Brown RS Jr, et al. Ledipasvir and sofosbuvir plus ribavirin for treatment of HCV infection in patients with advanced liver disease. Gastroenterology 2015;149:649-659.

113. Curry MP, O'Leary JG, Bzowej N, Muir AJ, Korenblat KM, Fenkel $J M$, et al. Sofosbuvir and velpatasvir for HCV in patients with decompensated cirrhosis. N Engl J Med 2015;373:2618-2628.

114. Foster GR, Irving WL, Cheung MC, Walker AJ, Hudson BE, Verma $S$, et al. Impact of direct acting antiviral therapy in patients with chronic hepatitis C and decompensated cirrhosis. J Hepatol 2016:64:1224-1231.

115. McCaughan G, Roberts SK, Strasser SI, Gow P, Wigg AJ, Tallis C, et al. The TOSCAR study: sofosbuvir and daclatasvir therapy for decompensted HCV cirrhosis with MELD score $>15$ : what is the point of no return? [Abstract]. Hepatology 2015;62:738A.

116. American Association for the Study of Liver Diseases/Infectious Diseases Society of America (AASLD/IDSA). HCV Guidance: Recommendations for Testing, Managing, and Treating Hepatitis C. AASLD web site, <http://www.hcvguidelines.org >. Accessed Sep 2017.

117. Terrault NA, Hassanein TI. Management of the patient with SVR [Abstract]. J Hepatol 2016;65(Suppl1):S120-S129.

118. Garcia-Retortillo M, Forns X, Feliu A, Moitinho E, Costa J, Navasa $M$, et al. Hepatitis $C$ virus kinetics during and immediately after liver transplantation. Hepatology 2002;35:680-687.

119. Forman LM, Lewis JD, Berlin JA, Feldman HI, Lucey MR. The association between hepatitis $C$ infection and survival after orthotopic liver transplantation. Gastroenterology 2002;122:889-896.

120. Gane EJ, Portmann BC, Naoumov NV, Smith HM, Underhill JA, Donaldson PT, et al. Long-term outcome of hepatitis C infection after liver transplantation. N Engl J Med 1996;334:815-820.

121. Curry MP, Forns $X$, Chung RT, Terrault NA, Brown R Jr, Fenkel JM, et al. Sofosbuvir and ribavirin prevent recurrence of HCV infection after liver transplantation: an open-label study. Gastroenterology 2015;148:100-107.e1.

122. Belli LS, Duvoux C, Berenguer M, Berg T, Coilly A, Colle I, et al. ELITA consensus statements on the use of DAAs in liver transplant candidates and recipients. J Hepatol 2017;67:585-602.

123. Manns M, Samuel D, Gane EJ, Mutimer D, McCaughan G, Buti M, et al. Ledipasvir and sofosbuvir plus ribavirin in patients with genotype 1 or 4 hepatitis $C$ virus infection and advanced liver disease: a multicentre, open-label, randomised, phase 2 trial. Lancet Infect Dis 2016;16:685-697.

124. Reau N, Kwo PY, Rhee S, Brown RS, Agarwal K, Angus P, et al. MAGELLAN-2: safety and efficacy of glecaprevir/pibrentasvir in liver or renal transplant adults with chronic hepatitis $C$ genotype 1-6 infection [Abstract]. J Hepatol 2017;66:S90-S91. 
The Korean Association for the Study of the Liver (KASL) 2017 KASL clinical practice guidelines: Management of chronic hepatitis C

125. Kwo PY, Mantry PS, Coakley E, Te HS, Vargas HE, Brown R Jr, et al. An interferon-free antiviral regimen for HCV after liver transplantation. N Engl J Med 2014;371:2375-2382.

126. European Association for the Study of the Liver. EASL Recommendations on treatment of hepatitis C 2016. J Hepatol 2017;66:153-194.

127. Martin P, Fabrizi F. Hepatitis C virus and kidney disease. J Hepatol 2008;49:613-624.

128. Fabrizi F, Lunghi G, Dixit V, Martin P. Meta-analysis: anti-viral therapy of hepatitis $C$ virus-related liver disease in renal transplant patients. Aliment Pharmacol Ther 2006;24:1413-1422.

129. Cholongitas E, Pipili C, Papatheodoridis GV. Interferon-free regimens in patients with hepatitis $C$ infection and renal dysfunction or kidney transplantation. World J Hepatol 2017;9:180.

130. Bravo MJ, Vallejo F, Barrio G, Brugal MT, Molist G, Pulido J, et al. $\mathrm{HCV}$ seroconversion among never-injecting heroin users at baseline: no predictors identified other than starting injection. Int J Drug Policy 2012;23:415-419.

131. Min JA, Yoon Y, Lee HJ, Choi J, Kwon M, Kim K, et al. Prevalence and associated clinical characteristics of hepatitis B, C, and HIV infections among injecting drug users in Korea. J Med Virol 2013;85:575-582.

132. Yun H, Kim D, Kim S, Kang S, Jeong S, Cheon Y, et al. High prevalence of HBV and HCV infection among intravenous drug users in Korea. J Med Virol 2008;80:1570-1575.

133. Dimova RB, Zeremski M, Jacobson IM, Hagan H, Des Jarlais DC, Talal AH. Determinants of hepatitis $\mathrm{C}$ virus treatment completion and efficacy in drug users assessed by meta-analysis. Clin Infect Dis 2013;56:806-816.

134. Lalezari J, Sullivan JG, Varunok P, Galen E, Kowdley KV, Rustgi V, et al. Ombitasvir/paritaprevir/r and dasabuvir plus ribavirin in $\mathrm{HCV}$ genotype 1-infected patients on methadone or buprenorphine. J Hepatol 2015;63:364-369.

135. Dore GJ, Altice F, Litwin AH, Dalgard O, Gane EJ, Shibolet O, et al. Elbasvir-grazoprevir to treat hepatitis $C$ virus infection in persons receiving opioid agonist therapy: a randomized trial. Ann Intern Med 2016;165:625-634.

136. Grebely J, Robaeys G, Bruggmann P, Aghemo A, Backmund M, Bruneau J, et al. Recommendations for the management of hepatitis $C$ virus infection among people who inject drugs. Int J Drug Policy 2015;26:1028-1038.

137. Garimella T, Wang R, Luo WL, Wastall P, Kandoussi H, DeMicco M, et al. Assessment of drug-drug interactions between daclatasvir and methadone or buprenorphine-naloxone. Antimicrob Agents Chemother 2015;59:5503-5510.

138. Menon RM, Badri PS, Wang T, Polepally AR, Zha J, Khatri A, et al. Drug-drug interaction profile of the all-oral anti-hepatitis $C$ virus regimen of paritaprevir/ritonavir, ombitasvir, and dasabuvir. J Hepatol 2015;63:20-29.
139. Smolders EJ, de Kanter CT, de Knegt RJ, van der Valk M, Drenth JP, Burger DM, et al. Drug-drug interactions between direct-acting antivirals and psychoactive medications. Clin Pharmacokinet 2016:55:1471-1494.

140. Liu CH, Kao JH. Treatment of hepatitis C virus infection in patients with end-stage renal disease. J Gastroenterol Hepatol 2011;26:228239.

141. Bang BK, Choi BS, Kim HW, Kim SK, Yang CW, Kim YS, et al. Retrospective study on the impact of hepatitis $B$ and hepatitis $C$ virus infection on renal transplnat recipients over 15 years. Korean J Nephrol 2002;21:423-434.

142. Shin YH, Kim HK, Choi SD, Kim YS, Shin HS, Won YJ, et al. Prevalence of Anti-HCV in hemodialysis patients in Taegu and Kyeongbuk, Korea. Korean J Med 1998;54:640-646.

143. Kim H, Kim KT, Yoo JH, Kim BI, Lee SJ, Lee EJ, et al. Prevalence and risk factors of hepatitis $\mathrm{C}$ virus infection in chronic hamodialysis patients(multi-center study). Korean J Med 1997;52:833-840.

144. Jin DC, Yun SR, Lee SW, Han SW, Kim W, Park J. Current characteristics of dialysis therapy in Korea: 2015 registry data focusing on elderly patients. Kidney Res Clin Pract 2016;35:204-211.

145. Kidney Disease: Improving Global Outcomes (KDIGO). KDIGO clinical practice guidelines for the prevention, diagnosis, evaluation, and treatment of hepatitis C in chronic kidney disease. Kidney Int Suppl 2008;(109):S1-S99.

146. Lee JJ, Lin MY, Chang JS, Hung CC, Chang JM, Chen HC, et al. Hepatitis $C$ virus infection increases risk of developing end-stage renal disease using competing risk analysis. PLoS One 2014;9:e100790.

147. Fabrizi F, Dixit V, Messa P. Impact of hepatitis $C$ on survival in dialysis patients: a link with cardiovascular mortality? J Viral Hepat 2012;19:601-607.

148. Aroldi A, Lampertico P, Montagnino G, Passerini P, Villa M, Campise MR, et al. Natural history of hepatitis $B$ and $C$ in renal allograft recipients. Transplantation 2005;79:1132-1136.

149. Bruchfeld A, Wilczek $H$, Elinder CG. Hepatitis C infection, time in renal-replacement therapy, and outcome after kidney transplantation. Transplantation 2004;78:745-750.

150. Choy BY, Chan TM, Lai KN. Recurrent glomerulonephritis after kidney transplantation. Am J Transplant 2006;6:2535-2542.

151. Kamar N, Mariat C, Delahousse M, Dantal J, Al Najjar A, Cassuto E, et al. Diabetes mellitus after kidney transplantation: a French multicentre observational study. Nephrol Dial Transplant 2007;22:19861993.

152. Pockros PJ, Reddy KR, Mantry PS, Cohen E, Bennett M, Sulkowski $M S$, et al. Efficacy of direct-acting antiviral combination for patients with hepatitis C virus genotype 1 infection and severe renal impairment or end-stage renal disease. Gastroenterology 2016;150:15901598.

153. Roth D, Nelson DR, Bruchfeld A, Liapakis A, Silva M, Monsour H Jr, 
et al. Grazoprevir plus elbasvir in treatment-naive and treatmentexperienced patients with hepatitis C virus genotype 1 infection and stage 4-5 chronic kidney disease (the C-SURFER study): a combination phase 3 study. Lancet 2015;386:1537-1545.

154. Pol S, Pockros PJ, Pugatch D, Brau N, Landis C, Elkhashab M, et al. Safety and efficacy of glecaprevir/pibrentasvir in adults with chronic hepatitis C virus infection genotype 1 and 6 and chronic kidney disease: an integrated analysis [Abstract]. Gastroenterology 2017;152:S1062-S1063.

155. Toyoda H, Kumada T, Tada T, Takaguchi K, Ishikawa T, Tsuji K, et al. Safety and efficacy of dual direct-acting antiviral therapy (daclatasvir and asunaprevir) for chronic hepatitis C virus genotype 1 infection in patients on hemodialysis. J Gastroenterol 2016;51:741747.

156. Bruchfeld A, Lindahl K, Reichard O, Carlsson T, Schvarcz R. Pegylated interferon and ribavirin treatment for hepatitis $C$ in haemodialysis patients. J Viral Hepat 2006;13:316-321.

157. Mazzaro C, Zorat F, Caizzi M, Donada C, Di Gennaro G, Maso LD, et al. Treatment with peg-interferon alfa-2b and ribavirin of hepatitis C virus-associated mixed cryoglobulinemia: a pilot study. J Hepatol 2005;42:632-638.

158. Saadoun D, Resche-Rigon M, Thibault V, Piette JC, Cacoub P. Antiviral therapy for hepatitis $C$ virus--associated mixed cryoglobulinemia vasculitis: a long-term followup study. Arthritis Rheum 2006;54:3696-3706.

159. Saadoun D, Pol S, Ferfar Y, Alric L, Hezode C, Si Ahmed SN, et al. Efficacy and safety of sofosbuvir plus daclatasvir for treatment of HCV-associated cryoglobulinemia vasculitis. Gastroenterology 2017;153:49-52.e5.

160. Sise ME, Bloom AK, Wisocky J, Lin MV, Gustafson JL, Lundquist $A L$, et al. Treatment of hepatitis $C$ virus-associated mixed cryoglobulinemia with direct-acting antiviral agents. Hepatology 2016;63:408-417.

161. Sherman KE, Rouster SD, Chung RT, Rajicic N. Hepatitis C virus prevalence among patients infected with human immunodeficiency virus: a cross-sectional analysis of the US adult AIDS clinical trials group. Clin Infect Dis 2002;34:831-837.

162. Lee SH, Kim KH, Lee SG, Chen DH, Jung DS, Moon CS, et al. Trends of mortality and cause of death among HIV-infected patients in Korea, 1990-2011. J Korean Med Sci 2013;28:67-73.

163. Lee SH, Kim KH, Lee SG, Cho H, Chen DH, Chung JS, et al. Causes of death and risk factors for mortality among HIV-infected patients receiving antiretroviral therapy in Korea. J Korean Med Sci 2013;28:990-997.

164. Bonacini M, Lin HJ, Hollinger FB. Effect of coexisting HIV-1 infection on the diagnosis and evaluation of hepatitis C virus. J Acquir Immune Defic Syndr 2001;26:340-344.

165. Bica I, McGovern B, Dhar R, Stone D, McGowan K, Scheib R, et al. Increasing mortality due to end-stage liver disease in patients with human immunodeficiency virus infection. Clin Infect Dis 2001;32:492-497.

166. Thomas DL, Astemborski J, Rai RM, Anania FA, Schaeffer M, Galai $\mathrm{N}$, et al. The natural history of hepatitis $C$ virus infection: host, viral, and environmental factors. JAMA 2000;284:450-456.

167. Torriani FJ, Rodriguez-Torres M, Rockstroh JK, Lissen E, GonzalezGarcía J, Lazzarin A, et al. Peginterferon Alfa-2a plus ribavirin for chronic hepatitis C virus infection in HIV-infected patients. N Engl J Med 2004;351:438-450.

168. Weber R, Sabin CA, Friis-Møller N, Reiss P, El-Sadr WM, Kirk $O$, et al. Liver-related deaths in persons infected with the human immunodeficiency virus: the D:A:D study. Arch Intern Med 2006;166:1632-1641.

169. Qurishi N, Kreuzberg C, Lüchters G, Effenberger W, Kupfer B, Sauerbruch $T$, et al. Effect of antiretroviral therapy on liver-related mortality in patients with HIV and hepatitis C virus coinfection. Lancet 2003;362:1708-1713.

170. Avidan NU, Goldstein D, Rozenberg L, McLaughlin M, Ferenci P, Masur $\mathrm{H}$, et al. Hepatitis $\mathrm{C}$ viral kinetics during treatment with peg IFN-alpha-2b in HIV/HCV coinfected patients as a function of baseline CD4+ T-cell counts. J Acquir Immune Defic Syndr 2009;52:452458.

171. Sulkowski MS, Mehta SH, Torbenson MS, Higgins Y, Brinkley SC, de Oca RM, et al. Rapid fibrosis progression among HIV/hepatitis C virus-co-infected adults. Aids 2007;21:2209-2216.

172. Tien PC; Veterans Affairs Hepatitis C Resource Center Program; National Hepatitis C Program Office. Management and treatment of hepatitis $\mathrm{C}$ virus infection in HIV-infected adults: recommendations from the Veterans Affairs Hepatitis C Resource Center Program and National Hepatitis C Program Office. Am J Gastroenterol 2005;100:2338-2354.

173. Bräu N, Salvatore M, Ríos-Bedoya CF, Fernández-Carbia A, Paronetto $F$, Rodríguez-Orengo JF, et al. Slower fibrosis progression in HIV/HCV-coinfected patients with successful HIV suppression using antiretroviral therapy. J Hepatol 2006;44:47-55.

174. Macías J, Berenguer J, Japón MA, Girón JA, Rivero A, López-Cortés $L F$, et al. Fast fibrosis progression between repeated liver biopsies in patients coinfected with human immunodeficiency virus/hepatitis C virus. Hepatology 2009;50:1056-1063.

175. The Korean Society for AIDS. Clinical guidelines for the diagnosis and treatment of HIVIAIDS in HIV-infected Koreans. Infect Chemother 2011:43:89-128.

176. DHHS Panel on Antiretroviral Guidelines for Adults and Adolescents - A Working Group of the Office of AIDS Research Advisory Council (OARAC). Guidelines for the Use of Antiretroviral Agents in HIV1-Infected Adults and Adolescents. AIDSinfo web site, <https:// aidsinfo.nih.gov/guidelines>. Accessed Sep 2017. 
The Korean Association for the Study of the Liver (KASL) 2017 KASL clinical practice guidelines: Management of chronic hepatitis C

177. Sulkowski MS. Management of acute and chronic HCV infection in persons with HIV coinfection. J Hepatol 2014;61(1 Suppl):S108S119.

178. Alvarez D, Dieterich DT, Brau N, Moorehead L, Ball L, Sulkowski MS. Zidovudine use but not weight-based ribavirin dosing impacts anaemia during HCV treatment in HIV-infected persons. J Viral Hepat 2006;13:683-689.

179. Lafeuillade A, Hittinger G, Chadapaud S. Increased mitochondrial toxicity with ribavirin in HIV/HCV coinfection. Lancet 2001;357:280-281.

180. Salmon-Céron D, Chauvelot-Moachon L, Abad S, Silbermann B, Sogni P. Mitochondrial toxic effects and ribavirin. Lancet 2001;357:1803-1804.

181. Osinusi A, Townsend K, Kohli A, Nelson A, Seamon C, Meissner $E G$, et al. Virologic response following combined ledipasvir and sofosbuvir administration in patients with HCV genotype 1 and HIV co-infection. JAMA 2015;313:1232-1239.

182. Sulkowski MS, Eron JJ, Wyles D, Trinh R, Lalezari J, Wang C, et al. Ombitasvir, paritaprevir co-dosed with ritonavir, dasabuvir, and ribavirin for hepatitis $C$ in patients co-infected with HIV-1: a randomized trial. JAMA 2015;313:1223-1231.

183. Naggie S, Cooper C, Saag M, Workowski K, Ruane P, Towner WJ, et al. Ledipasvir and sofosbuvir for HCV in patients coinfected with HIV-1. N Engl J Med 2015;373:705-713.

184. Molina JM, Orkin C, Iser DM, Zamora FX, Nelson M, Stephan C, et al. Sofosbuvir plus ribavirin for treatment of hepatitis $C$ virus in patients co-infected with HIV (PHOTON-2): a multicentre, open-label, non-randomised, phase 3 study. Lancet 2015;385:1098-1106.

185. Fernández-Montero JV, Soriano V. Management of hepatitis C in HIV and/or HBV co-infected patients. Best Pract Res Clin Gastroenterol 2012;26:517-530.

186. Kim YJ, Lee JW, Kim YS, Jeong SH, Kim YS, Yim HJ, et al. Clinical features and treatment efficacy of peginterferon alfa plus ribavirin in chronic hepatitis C patients coinfected with hepatitis B virus. Korean J Hepatol 2011;17:199-205.

187. Chiaramonte M, Stroffolini T, Vian A, Stazi MA, Floreani A, Lorenzoni $U$, et al. Rate of incidence of hepatocellular carcinoma in patients with compensated viral cirrhosis. Cancer 1999;85:21322137.

188. Lee LP, Dai CY, Chuang WL, Chang WY, Hou NJ, Hsieh MY, et al. Comparison of liver histopathology between chronic hepatitis $C$ patients and chronic hepatitis B and C-coinfected patients. J Gastroenterol Hepatol 2007;22:515-517.

189. Sagnelli E, Pasquale G, Coppola N, Scarano F, Marrocco C, Scolastico $C$, et al. Influence of chronic coinfection with hepatitis B and $C$ virus on liver histology. Infection 2004;32:144-148.

190. Liu CJ, Chuang WL, Lee CM, Yu ML, Lu SN, Wu SS, et al. Peginterferon alfa-2a plus ribavirin for the treatment of dual chronic infection with hepatitis B and C viruses. Gastroenterology 2009;136:496-504.e3.

191. Potthoff A, Wedemeyer H, Boecher WO, Berg T, Zeuzem S, Arnold J, et al. The HEP-NET B/C co-infection trial: a prospective multicenter study to investigate the efficacy of pegylated interferon-alpha2b and ribavirin in patients with HBV/HCV co-infection. J Hepatol 2008;49:688-694.

192. Collins JM, Raphael KL, Terry C, Cartwright EJ, Pillai A, Anania FA, et al. Hepatitis $B$ virus reactivation during successful treatment of hepatitis $C$ virus with sofosbuvir and simeprevir. Clin Infect Dis 2015;61:1304-1306

193. Potthoff A, Berg T, Wedemeyer H; HEP-NET B/C Coinfection Study Group. Late hepatitis B virus relapse in patients co-infected with hepatitis B virus and hepatitis C virus after antiviral treatment with pegylated interferon-a2b and ribavirin. Scand J Gastroenterol 2009;44:1487-1490.

194. Belperio PS, Shahoumian TA, Mole LA, Backus LI. Evaluation of hepatitis $B$ reactivation among 62,920 veterans treated with oral hepatitis C antivirals. Hepatology 2017;66:27-36.

195. Bersoff-Matcha SJ, Cao K, Jason M, Ajao A, Jones SC, Meyer T, et al. Hepatitis $B$ virus reactivation associated with direct-acting antiviral therapy for chronic hepatitis $C$ virus: a review of cases reported to the U.S. Food and Drug Administration adverse event reporting system. Ann Intern Med 2017;166:792-798.

196. Chen G, Wang C, Chen J, Ji D, Wang Y, Wu V, et al. Hepatitis B reactivation in hepatitis $B$ and $C$ coinfected patients treated with antiviral agents: a systematic review and meta-analysis. Hepatology 2017;66:13-26.

197. Omata M, Kanda T, Wei L, Yu ML, Chuang WL, Ibrahim A, et al. APASL consensus statements and recommendations for hepatitis C prevention, epidemiology, and laboratory testing. Hepatol Int 2016;10:681-701.

198. Plug I, Van Der Bom JG, Peters M, Mauser-Bunschoten EP, De Goede-Bolder A, Heijnen $L$, et al. Mortality and causes of death in patients with hemophilia, 1992-2001: a prospective cohort study. J Thromb Haemost 2006;4:510-516.

199. Vento S, Cainelli F, Cesario F. Infections and thalassaemia. Lancet Infect Dis 2006;6:226-233.

200. Zhang M, Rosenberg PS, Brown DL, Preiss L, Konkle BA, Eyster $M E$, et al. Correlates of spontaneous clearance of hepatitis $C$ virus among people with hemophilia. Blood 2006;107:892-897.

201. Hézode C, Colombo M, Bourlière M, Spengler U, Ben-Ari Z, Strasser $\mathrm{SI}$, et al. Elbasvir/grazoprevir for patients with hepatitis $C$ virus infection and inherited blood disorders: a phase III study. Hepatology 2017;66:736-745

202. Ozguroglu M, Bilici A, Turna $H$, Serdengecti S. Reactivation of hepatitis $B$ virus infection with cytotoxic therapy in non-Hodgkin's lymphoma. Med Oncol 2004;21:67-72. 
203. Kawatani T, Suou T, Tajima F, Ishiga $K$, Omura $H$, Endo A, et al. Incidence of hepatitis virus infection and severe liver dysfunction in patients receiving chemotherapy for hematologic malignancies. Eur J Haematol 2001;67:45-50.

204. Markovic S, Drozina G, Vovk M, Fidler-Jenko M. Reactivation of hepatitis $B$ but not hepatitis $C$ in patients with malignant lymphoma and immunosuppressive therapy. A prospective study in 305 patients. Hepatogastroenterology 1999;46:2925-2930.

205. Vento $S$, Cainelli F, Longhi MS. Reactivation of replication of hepatitis $B$ and $C$ viruses after immunosuppressive therapy: an unresolved issue. Lancet Oncol 2002;3:333-340.

206. Faggioli P, De Paschale M, Tocci A, Luoni M, Fava S, De Paoli A, et al. Acute hepatic toxicity during cyclic chemotherapy in non Hodgkin's lymphoma. Haematologica 1997;82:38-42.

207. Nosotti L, D'Andrea M, Pitidis A, Pimpinelli F, Dessanti ML, Pisani F, et al. Hepatitis $C$ virus infection prevalence and liver dysfunction in a cohort of B-cell non-Hodgkin's lymphoma patients treated with immunochemotherapy. Scand J Infect Dis 2012;44:70-73.

208. Takai S, Tsurumi H, Ando K, Kasahara S, Sawada M, Yamada T, et al. Prevalence of hepatitis $B$ and $C$ virus infection in haematological malignancies and liver injury following chemotherapy. Eur J Haematol 2005;74:158-165.

209. Fan FS, Tzeng CH, Hsiao KI, Hu ST, Liu WT, Chen PM. Withdrawal of immunosuppressive therapy in allogeneic bone marrow transplantation reactivates chronic viral hepatitis C. Bone Marrow Transplant 1991;8:417-420.

210. Melisko ME, Fox $R$, Venook $A$. Reactivation of hepatitis $C$ virus after chemotherapy for colon cancer. Clin Oncol (R Coll Radiol) 2004;16:204-205.

211. Vento S, Cainelli F, Mirandola F, Cosco L, Di Perri G, Solbiati M, et al. Fulminant hepatitis on withdrawal of chemotherapy in carriers of hepatitis C virus. Lancet 1996;347:92-93.

212. Hamaguchi M, Yamada H, Gondo H, Takemoto Y, Morishima $Y$, Kodera $Y$. Retrospective study on the impact of hepatitis $B$ and hepatitis C virus infection on hematopoietic stem cell transplantation in Japan. Int J Hematol 2002;75:324-331.

213. Locasciulli A, Bruno B, Alessandrino EP, Meloni G, Arcese W, Ban$\operatorname{dini} \mathrm{G}$, et al. Hepatitis reactivation and liver failure in haemopoietic stem cell transplants for hepatitis B virus (HBV)/hepatitis C virus ( $\mathrm{HCV}$ ) positive recipients: a retrospective study by the Italian group for blood and marrow transplantation. Bone Marrow Transplant 2003;31:295-300.

214. Lee JM, Lee JM, Yoo HS, Jang UK, Kim DJ, Kim YB, et al. The prevalence of anti-HCV positivity in healthy Korean children. Korean J Hepatol 1996:2:160-165.

215. Mohan P, Barton BA, Narkewicz MR, Molleston JP, Gonzalez-Peralta RP, Rosenthal $\mathrm{P}$, et al. Evaluating progression of liver disease from repeat liver biopsies in children with chronic hepatitis C: a retrospective study. Hepatology 2013;58:1580-1586.

216. Okamoto M, Nagata I, Murakami J, Kaji S, litsuka T, Hoshika T, et al. Prospective reevaluation of risk factors in mother-to-child transmission of hepatitis C virus: high virus load, vaginal delivery, and negative anti-NS4 antibody. J Infect Dis 2000;182:1511-1514.

217. Claret G, Noguera A, Esteva C, Muñoz-Almagro C, Sánchez E, Fortuny $C$. Mother-to-child transmission of hepatitis $C$ virus infection in Barcelona, Spain: a prospective study. Eur J Pediatr 2007;166:1297-1299.

218. Kang MJ, Kim HJ, Park KJ, Kang KH, Ahn HS. Prevalence of HCV infection in pregnant women and vertical transmission. Korean J Obstet Gynecol 2004;47:2045-2050.

219. Kim YW, Lee JM, Kim GJ, Lee HM, Kim SY, Lee JS, et al. Hepatitis C virus infection in pregnancy. Korean J Obstet Gynecol 2000;43:597603.

220. Pembrey L, Newell ML, Tovo PA; EPHN Collaborators. The management of HCV infected pregnant women and their children European paediatric HCV network. J Hepatol 2005;43:515-525.

221. Hepatitis $C$ virus infection. American Academy of Pediatrics. Committee on Infectious Diseases. Pediatrics 1998;101:481-485.

222. Mast EE, Hwang LY, Seto DS, Nolte FS, Nainan OV, Wurtzel H, et al. Risk factors for perinatal transmission of hepatitis C virus (HCV) and the natural history of HCV infection acquired in infancy. J Infect Dis 2005;192:1880-1889.

223. Polywka S, Pembrey L, Tovo PA, Newell ML. Accuracy of HCV-RNA $P C R$ tests for diagnosis or exclusion of vertically acquired HCV infection. J Med Virol 2006;78:305-310.

224. Guido M, Rugge M, Jara P, Hierro L, Giacchino R, Larrauri J, et al. Chronic hepatitis $C$ in children: the pathological and clinical spectrum. Gastroenterology 1998;115:1525-1529.

225. Mack CL, Gonzalez-Peralta RP, Gupta N, Leung D, Narkewicz MR, Roberts EA, et al. NASPGHAN practice guidelines: diagnosis and management of hepatitis $C$ infection in infants, children, and adolescents. J Pediatr Gastroenterol Nutr 2012;54:838-855.

226. Balistreri WF, Murray KF, Rosenthal P, Bansal S, Lin CH, Kersey K, et al. The safety and effectiveness of ledipasvir-sofosbuvir in adolescents 12-17 years old with hepatitis C virus genotype 1 infection. Hepatology 2017;66:371-378.

227. Wirth S, Pieper-Boustani H, Lang T, Ballauff A, Kullmer U, Gerner P, et al. Peginterferon alfa-2b plus ribavirin treatment in children and adolescents with chronic hepatitis C. Hepatology 2005;41:10131018.

228. Wirth S, Ribes-Koninckx C, Calzado MA, Bortolotti F, Zancan L, Jara $P$, et al. High sustained virologic response rates in children with chronic hepatitis $C$ receiving peginterferon alfa-2b plus ribavirin. J Hepatol 2010;52:501-507.

229. Schwarz KB, Gonzalez-Peralta RP, Murray KF, Molleston JP, Haber $\mathrm{BA}$, Jonas $\mathrm{MM}$, et al. The combination of ribavirin and peginterfer- 
The Korean Association for the Study of the Liver (KASL) 2017 KASL clinical practice guidelines: Management of chronic hepatitis C

on is superior to peginterferon and placebo for children and adolescents with chronic hepatitis C. Gastroenterology 2011;140:450458.e1.

230. Akuta N, Sezaki H, Suzuki F, Fujiyama S, Kawamura Y, Hosaka $\mathrm{T}$, et al. Ledipasvir plus sofosbuvir as salvage therapy for HCV genotype 1 failures to prior NS5A inhibitors regimens. J Med Virol 2017;89:1248-1254.

231. Lawitz E, Flamm SL, Yang JC, Pang PS, Zhu Y, Svarovskaia E, et al. Retreatment of patients who failed 8 or 12 weeks of ledipasvir/ sofosbuvir-based regimens with ledipasvir/sofosbuvir for 24 weeks [Abstract]. J Hepatol 2015;62 (Suppl 2):S192.

232. Lawitz E, Poordad F, Gutierrez JA, Wells JT, Landaverde CE, Evans B, et al. Short-duration treatment with elbasvir/grazoprevir and sofosbuvir for hepatitis C: a randomized trial. Hepatology 2017;65:439450.

233. Poordad F, Bennett M, Sepe TE, Cohen E, Reindollar RW, Everson $\mathrm{G}$, et al. Ombitasvir/paritaprevir/r, dasabuvir, and sofosbuvir treatment of patients with HCV genotype 1-infection who failed a prior course of DAA therapy: the quartz-I study. J Hepatol 2016;64:S767S768.

234. Bourlière M, Gordon SC, Flamm SL, Cooper CL, Ramji A, Tong M, et al. Sofosbuvir, velpatasvir, and voxilaprevir for previously treated HCV infection. N Engl J Med 2017;376:2134-2146.

235. Gane EJ, Shiffman ML, Etzkorn K, Morelli G, Stedman CAM, Davis $M N$, et al. Sofosbuvir-velpatasvir with ribavirin for 24 weeks in hepatitis C virus patients previously treated with a direct-acting antiviral regimen. Hepatology 2017;66:1083-1089.

236. Poordad F, Felizarta F, Asatryan A, Sulkowski MS, Reindollar RW, Landis CS, et al. Glecaprevir and pibrentasvir for 12 weeks for hepatitis $C$ virus genotype 1 infection and prior direct-acting antiviral treatment. Hepatology 2017;66:389-397.

237. Poordad F, Pol S, Asatryan A, Buti M, Shaw D, Hézode C, et al. MAGELLAN-1, Part 2: glecaprevir and pibrentasvir for 12 or 16 weeks in patients with chronic hepatitis C virus genotype 1 or 4 and prior direct-acting antiviral treatment failure [Abstract]. J Hepatol 2017;66:S83-S84.

238. Pilot-Matis T, Krishnan P, Schnell G, Tripathi R, Beyer J, Reisch T, et al. Resistance analysis in the MAGELLAN-1 Study (Part 2): glecaprevir/pibrentasvir therapy in $\mathrm{HCV}$-infected patients who had failed prior DAA regimens containing NS3/4A protease and/or NS5A inhibirots [Abstract]. J Hepatol 2017;66:S708-S709.

239. Osinusi A, Kohli A, Marti MM, Nelson A, Zhang X, Meissner EG, et al. Re-treatment of chronic hepatitis $C$ virus genotype 1 infection after relapse: an open-label pilot study. Ann Intern Med 2014;161:634-638.

240. Wyles D, Pockros P, Morelli G, Younes Z, Svarovskaia E, Yang JC, et al. Ledipasvir-sofosbuvir plus ribavirin for patients with genotype 1 hepatitis C virus previously treated in clinical trials of sofosbuvir regimens. Hepatology 2015;61:1793-1797.

241. Hassanein T, Wyles D, Wang S, Kwo PY, Shiffman ML, Younes Z, et al. SURVEYOR-II, part 4: glecaprevir/pibrentasvir demonstrates high SVR rates in patients with HCV genotype 2, 4, 5, or 6 infection without cirrhosis following an 8-week treatment duration [Abstract]. Hepatology 2016;64:1128A.

242. Kowdley KV, Colombo M, Zadeikis N, Mantry PS, Calinas F, Aguilar H. ENDURANCE-2: safety and efficacy of glecaprevir/pibrentasvir in hepatitis $C$ virus genotype 2-infected patients without cirrhosis: a randomized, double-blind, placebo-controlled study [Abstract]. Hepatology 2016;64(Suppl 1):39A. 